\title{
Graded Lie Superalgebras, Supertrace Formula, and Orbit Lie Superalgebras
}

\author{
Seok-Jin Kang* and Jae-Hoon Kwon ${ }^{\diamond}$ \\ *Department of Mathematics \\ Seoul National University \\ Seoul 151-742, Korea \\ ${ }^{\diamond}$ Department of Mathematics \\ Korea Air Force Academy \\ Chong-Ju 363-849, Korea \\ June 17, 2022
}

\begin{abstract}
Let $\Gamma$ be a countable abelian semigroup and $\mathcal{A}$ be a countable abelian group satisfying a certain finiteness condition. Suppose that a group $G$ acts on a $(\Gamma \times \mathcal{A})$-graded Lie superalgebra $\mathfrak{L}=\bigoplus_{(\alpha, a) \in \Gamma \times \mathcal{A}} \mathfrak{L}_{(\alpha, a)}$ by Lie superalgebra automorphisms preserving the $(\Gamma \times \mathcal{A})$-gradation. In this paper, we show that the Euler-Poincaré principle yields the generalized denominator identity for $\mathfrak{L}$ and derive a closed form formula for the supertraces $\operatorname{str}\left(g \mid \mathfrak{L}_{(\alpha, a)}\right)$ for all $g \in G$, $(\alpha, a) \in \Gamma \times \mathcal{A}$. We discuss the applications of our supertrace formula to various classes of infinite dimensional Lie superalgebras such as free Lie superalgebras and generalized Kac-Moody superalgebras. In particular, we determine the decomposition of free Lie superalgebras into a direct sum of irreducible $G L(n) \times G L(k)$ modules, and the supertraces of the Monstrous Lie superalgebras with group actions. Finally, we prove that the generalized characters of Verma modules and the irreducible highest weight modules over a generalized Kac-Moody superalgebra $\mathfrak{g}$ corresponding to the Dynkin diagram automorphism $\sigma$ are the same as the usual characters of Verma modules and irreducible highest weight modules over the orbit Lie superalgebra $\breve{\mathfrak{g}}=\mathfrak{g}(\sigma)$ determined by $\sigma$.
\end{abstract}

*Supported in part by Basic Science Research Institute Program, Ministry of Education of Korea, BSRI-98-1414, and GARC-KOSEF at Seoul National University. 


\section{Introduction}

In the past three decades, the theory of infinite dimensional Lie algebras and their representations has been the focus of extensive research activities due to its rich and significant applications to many areas of mathematics and mathematical physics. The most well-known examples are Kac-Moody algebras ([27], [48]) and generalized KacMoody algebras ([5]), and a lot of exciting new discoveries have been made using the language of these infinite dimensional Lie algebras. For example, the Macdonald identities ([45]) were shown to be equivalent to the denominator identities of affine KacMoody algebras (28]), and the Moonshine Conjecture was proved by investigating the structure of a special kind of generalized Kac-Moody algebra called the Monster Lie algebra $([6])$.

On the other hand, since 1970's, the Lie superalgebras and their representations have emerged naturally as the fundamental algebraic structure behind several areas of mathematical physics. In [29], V. G. Kac gave a comprehensive presentation of the mathematical theory of Lie superalgebras, and obtained an important classification theorem for finite dimensional simple Lie superalgebras over algebraically closed fields of characteristic zero (see also [53]). The theories of Kac-Moody algebras and generalized Kac-Moody algebras have been extended to those of Kac-Moody superalgebras ([30]) and generalized Kac-Moody superalgebras (447, [51]) following the outline given in [31]. In [34] and [35, V. G. Kac and M. Wakimoto developed the representation theory of affine Kac-Moody superalgebras, and demonstrated some interesting applications of affine Kac-Moody superalgebras to number theory.

In [37, S.-J. Kang derived a closed form root multiplicity formula for generalized Kac-Moody algebras, and by applying his formula to the Monster Lie algebra, he obtained some interesting recursive relations among the coefficients of the elliptic modular function $j$. The main idea of [37] is that the Euler-Poincaré principle for the Lie algebra homology can be interpreted as the denominator identity for Kac-Moody algebras or generalized Kac-Moody algebras, and once we are given the denominator identity, we can derive a root multiplicity formula. This idea has been extended to the general infinite dimensional graded Lie algebras to yield a closed form dimension formula for the homogeneous subspaces (42]), and a lot of interesting applications of our formula to various classes of infinite dimensional Lie algebras have been investigated (see also [38] and [41]). In [32, V. G. Kac and S.-J. Kang exploited this idea further to derive a closed form trace formula for graded Lie algebras with group actions. In particular, by applying their trace formula to the Monster simple group acting on the Monster Lie algebra, they obtained a family of interesting recursive relations among the coefficients of the Thompson series.

The Thompson series are examples of replicable functions which are normalized $q$ series satisfying certain functional equations called the replication formulae. In [40, S.-J. Kang showed that, given the denominator identity for graded Lie superalgebras, 
one can derive a closed form superdimension formula for the homogeneous subspaces, which can be considered as a unifying method of studying the structure of infinite dimensional Lie superalgebras. He discussed a lot of interesting applications of our superdimension formula to various classes of infinite dimensional Lie superalgebras such as free Lie superalgebras, generalized Kac-Moody superalgebras, and Monstrous Lie superalgebras. Furthermore, he characterized the replicable functions in terms of certain product identities, and determined the root multiplicities of the Monstrous Lie superalgebras associated with replicable functions.

In this paper, we combine the main ideas of [32] and [40] to derive a closed form supertrace formula for graded Lie superalgebras with group actions. More precisely, let $\Gamma$ be a countable (ususally infinite) abelian semigroup and $\mathcal{A}$ be a countable (usually finite) abelian group such that every element $(\alpha, a) \in \Gamma \times \mathcal{A}$ can be written as a sum of elements in $\Gamma \times \mathcal{A}$ in only finitely many ways. Suppose that a group $G$ acts on a graded Lie superalgebra $\mathfrak{L}=\bigoplus_{(\alpha, a) \in \Gamma \times \mathcal{A}} \mathfrak{L}_{(\alpha, a)}$ by Lie superalgebra automorphisms preserving the $(\Gamma \times \mathcal{A})$-gradation. Then, in Section 2, we show that the Euler-Poincaré principle for the Lie superalgebra homology yields a product identity

$$
\begin{array}{r}
\prod_{(\alpha, a) \in \Gamma \times \mathcal{A}} \exp \left(-\sum_{k=1}^{\infty} \frac{1}{k} \operatorname{str}\left(g^{k} \mid \mathfrak{L}_{(\alpha, a)}\right) E^{k(\alpha, a)}\right) \\
=1-\sum_{(\alpha, a) \in \Gamma \times \mathcal{A}} \operatorname{str}\left(g \mid H(\mathfrak{L})_{(\alpha, a)}\right) E^{(\alpha, a)},
\end{array}
$$

which is called the generalized denominator identity for $g \in G$. By taking the logarithm of both sides of the generalized denominator identity and using Möbius inversion, we derive a closed form formula for the supertraces $\operatorname{str}\left(g \mid \mathfrak{L}_{(\alpha, a)}\right)=\psi(a) \operatorname{tr}\left(g \mid \mathfrak{L}_{(\alpha, a)}\right)$ for all $g \in G,(\alpha, a) \in \Gamma \times \mathcal{A}$ (Theorem 2.4):

$$
\operatorname{str}\left(g \mid \mathfrak{L}_{(\alpha, a)}\right)=\sum_{\substack{d>0 \\(\alpha, a)=d(\tau, b)}} \frac{1}{d} \mu(d) W_{g^{d}}(\tau, b),
$$

where $\psi(a)$ is the sign map defined on $\mathcal{A}$ and $W_{g}(\tau, b)$ is the Witt partition function defined by (2.16).

In Section 3, we present the standard homology theory for Lie superalgebras. That is, we show that there exists a super-analogue of Koszul's complex for Lie superalgebras (Proposition 3.1). The $k$-th homology module $H_{k}(\mathfrak{L}, V)$ of the Lie superalgebra $\mathfrak{L}$ with coefficients in $V$ is defined by

$$
H_{k}(\mathfrak{L}, V)=\operatorname{Tor}_{k}^{U}(V, \mathbb{C})=H_{k}\left(V \otimes_{U} M\right)
$$

where $M=\left(M_{k}, \partial_{k}\right)$ is the super-analogue of Koszul's complex given by (3.1) and (3.2), and $U=U(\mathfrak{L})$ denotes the universal enveloping algebra of $\mathfrak{L}$. In particular, if $\mathfrak{L}(V)$ is the free Lie superalgebra generated by a superspace $V$, then we have $H_{1}(\mathfrak{L}(V))=V$ 
and $H_{k}(\mathfrak{L}(V))=0$ for all $k \geq 2$. Even though our presentation is a straightforward generalization of the homology theory of Lie algebras (cf. [10], [15]), we decided to include the detailed treatment here, for we could not find it in any other literature.

Section 4 and Section 5 are devoted to the applications of our supertrace formula to free Lie superalgebras. In Section 4, we generalize the classical Witt formula for free Lie algebras to obtain a closed form supertrace formula for free Lie superalgebras with group actions. As an application, we compare the structure of the free Lie algebras and free Lie superalgebras generated by the same group representations (Proposition 4.2). In Section 5, we consider the free Lie superalgebra $\mathfrak{L}(V)$ generated by the natural $(k+l)$-dimensional representation $V$ of the general linear Lie superalgebra $g l(k, l)$, and determine the decomposition of $\mathfrak{L}(V)$ into a direct sum of irreducible $G L(k) \times G L(l)$ modules (Proposition 5.3 and Proposition 5.4). The Hook Schur functions and the Littlewood-Richardson coefficients are the key ingredients of the decomposition.

In Section 6 and Section 7, we give a conjectural Kostant-type formula for the homology of the negative part of generalized Kac-Moody superalgebras, and derive a closed form supertrace formula for generalized Kac-Moody superalgebras with group actions preserving the root space decomposition (Theorem 6.5). Moreover, we apply our supertrace formula to determine the supertraces of the Monstrous Lie superalgebras with group actions preserving the root space decomposition (Proposition 7.1 and Corollary 7.2). We believe that the product identities characterizing the replicable functions ([40]) can be understood most naturally as the generalized denominator identities of some Mosntrous Lie superalgebra with a suitable group action.

In Section 8 and Section 9, we prove that the generalized characters of Verma modules and irreducible highest weight modules over a generalized Kac-Moody superalgebra $\mathfrak{g}$ corresponding to the Dynkin diagram automorphism $\sigma$ are the same as the usual characters of Verma modules and irreducible highest weight modules over the orbit Lie superalgebra $\breve{\mathfrak{g}}=\mathfrak{g}(\sigma)$ determined by $\sigma$ (Theorem 9.3 and Corollary 9.4). This fact was first proved in [13 for symmetrizable Kac-Moody algebras under the linking condition in solving the fixed point problem in conformal field theory and was generalized to generalized Kac-Moody algebras without the linking condition in [14. In this paper, we also show that the generalized denominator identity for a generalized Kac-Moody superalgebra $\mathfrak{g}$ for the Dynkin diagram automorphism $\sigma$ is the same as the usual denominator identity for the orbit Lie superalgebra $\breve{\mathfrak{g}}=\mathfrak{g}(\sigma)$ (Theorem 9.5). Our argument for generalized Kac-Moody superalgebras is similar to those in [13 and [14]. We observe that the generalized character of a Verma module can be written as the product part of a generalized denominator identity and derive an explicit formula for the traces of $\sigma$ on each invariant subspaces (which are not the same as root spaces in general) of $\mathfrak{g}$ in terms of the dimensions of some orbit Lie superalgebras. Therefore, we obtain a dimension formula for each homogeneous subspaces of the invariant subalgebra, which is also a generalized Kac-Moody superalgebra with a natural grading 
arising from $\sigma$ (Proposition 9.6).

Acknowledgments. The authors would like to express their sincere gratitude to Professor Victor G. Kac for his interest in this work and many valuable discussions.

\section{Graded Lie Superalgebras and Supertrace For- mula}

We recall some basic facts about Lie superalgebras ([29], [40], [53]). Let $\mathcal{A}$ be a countable abelian group and suppose we have a bimultiplicative map $\theta: \mathcal{A} \times \mathcal{A} \longrightarrow \mathbb{C}^{\times}$ satisfying

$$
\begin{aligned}
& \theta(a+b, c)=\theta(a, c) \theta(b, c), \\
& \theta(a, b+c)=\theta(a, b) \theta(a, c), \\
& \theta(a, b) \theta(b, a)=1 \text { for all } a, b \in \mathcal{A} .
\end{aligned}
$$

In particular, we have $\theta(a, a)= \pm 1$ for all $a \in \mathcal{A}$. The map $\theta$ satisfying the condition $(2.1)$ is called a coloring map on $\mathcal{A}$. Define a map $\psi: \mathcal{A} \longrightarrow\{ \pm 1\}$ by $\psi(a)=\theta(a, a)$. Then $\psi$ is a well-defined group homomorphism called the sign map on $\mathcal{A}$ with respect to $\theta$. Let $\mathcal{A}_{0}=\{a \in \mathcal{A} \mid \psi(a)=1\}$ and $\mathcal{A}_{1}=\{a \in \mathcal{A} \mid \psi(a)=-1\}$. Then we have a decomposition $\mathcal{A}=\mathcal{A}_{0} \cup \mathcal{A}_{1}$ and the elements of $\mathcal{A}$ in $\mathcal{A}_{0}$ (resp. $\mathcal{A}_{1}$ ) are called even (resp. odd).

A $\theta$-colored superspace is a pair $(V, \theta)$, where $V=\bigoplus_{a \in \mathcal{A}} V_{a}$ is an $\mathcal{A}$-graded vector space and $\theta: \mathcal{A} \times \mathcal{A} \rightarrow \mathbb{C}^{\times}$is a coloring map on $\mathcal{A}$. The elements of $V_{a}$ are called even (resp. odd) if $\psi(a)=1$ (resp. $\psi(a)=-1$ ). For each $a \in \mathcal{A}$, we define the superdimension of $V_{a}$ to be

$$
\operatorname{sdim} V_{a}=\psi(a) \operatorname{dim} V_{a} .
$$

Similarly, we define a $\theta$-colored superalgebra to be a pair $(U, \theta)$, where $U=\bigoplus_{a \in \mathcal{A}} U_{a}$ is an $\mathcal{A}$-graded associative algebra (i.e., $U_{a} U_{b} \subset U_{a+b}$ for all $a, b \in \mathcal{A}$ ) and $\theta: \mathcal{A} \times \mathcal{A} \rightarrow$ $\mathrm{C}^{\times}$is a coloring map on $\mathcal{A}$. The direct sum of $\mathcal{A}$-graded superalgebras is defined in the usual way, but, for $\theta$-colored superalgebras $U=\bigoplus_{a \in \mathcal{A}} U_{a}$ and $U^{\prime}=\bigoplus_{a^{\prime} \in \mathcal{A}} U_{a}^{\prime}$, we define the tensor product of $U$ and $U^{\prime}$ to be the $\theta$-colored superspace $U \otimes U^{\prime}$ with the natural $\mathcal{A}$-gradation

$$
\left(U \otimes U^{\prime}\right)_{a+a^{\prime}}=\operatorname{Span}\left\{u \otimes u^{\prime} \mid u \in U_{a}, u^{\prime} \in U_{a^{\prime}}^{\prime}\right\}
$$

and the multiplication given by

$$
\left(u \otimes u^{\prime}\right)\left(v \otimes v^{\prime}\right)=\theta\left(a^{\prime}, b\right)\left(u v \otimes u^{\prime} v^{\prime}\right)
$$


for $u \in U_{a}, v \in U_{b}, u^{\prime} \in U_{a^{\prime}}^{\prime}, v^{\prime} \in U_{b^{\prime}}^{\prime}, a, a^{\prime}, b, b^{\prime} \in \mathcal{A}$.

Definition 2.1 A $\theta$-colored Lie superalgebra is a $\theta$-colored superspace $\mathfrak{L}=\bigoplus_{a \in \mathcal{A}} \mathfrak{L}_{a}$ together with a bilinear operation $[]:, \mathfrak{L} \times \mathfrak{L} \rightarrow \mathfrak{L}$ satisfying

$$
\begin{aligned}
& {\left[\mathfrak{L}_{a}, \mathfrak{L}_{b}\right] \subset \mathfrak{L}_{a+b},} \\
& {[x, y]=-\theta(a, b)[y, x]} \\
& {[x,[y, z]]=[[x, y], z]+\theta(a, b)[y,[x, z]]}
\end{aligned}
$$

for all $x \in \mathfrak{L}_{a}, y \in \mathfrak{L}_{b}, z \in \mathfrak{L}$ and $a, b \in \mathcal{A}$.

Let $\mathfrak{L}_{0}=\bigoplus_{a \in \mathcal{A}_{0}} \mathfrak{L}_{a}$ and $\mathfrak{L}_{1}=\bigoplus_{b \in \mathcal{A}_{1}} \mathfrak{L}_{b}$. Then we have a decomposition $\mathfrak{L}=$ $\mathfrak{L}_{0} \oplus \mathfrak{L}_{1}$, and the homogeneous elements of $\mathfrak{L}_{0}$ (resp. $\mathfrak{L}_{1}$ ) are called even (resp. odd).

The universal enveloping algebra of a $\theta$-colored Lie superalgebra $\mathfrak{L}=\bigoplus_{a \in \mathcal{A}} \mathfrak{L}_{a}$ is the pair $(U(\mathfrak{L}), \iota)$, where $U(\mathfrak{L})$ is a $\theta$-colored superalgebra and $\iota: \mathfrak{L} \rightarrow U(\mathfrak{L})$ is a linear mapping satisfying

$$
\iota([x, y])=\iota(x) \iota(y)-\theta(a, b) \iota(y) \iota(x) \text { for } x \in \mathfrak{L}_{a}, y \in \mathfrak{L}_{b}
$$

such that for any $\theta$-colored superalgebra $U=\bigoplus_{a \in \mathcal{A}} U_{a}$ and a linear mapping $j: \mathfrak{L} \rightarrow U$ satisfying

$$
j([x, y])=j(x) j(y)-\theta(a, b) j(y) j(x) \text { for } x \in \mathfrak{L}_{a}, y \in \mathfrak{L}_{b},
$$

there exits a unique homomorphism $\psi: U(\mathfrak{L}) \rightarrow U$ of $\theta$-colored superalgebras satisfying $\psi \circ \iota=j$.

The uniqueness of the universal enveloping algebra $U(\mathfrak{L})$ can be proved in the usual way. For the existence, consider the tensor algebra $\mathcal{T}(\mathfrak{L})=\bigoplus_{k=0}^{\infty} \mathfrak{L} \otimes k$ of $\mathfrak{L}$ and let $\mathcal{R}$ be the ideal of $\mathcal{T}(\mathfrak{L})$ generated by the elements of the form

$$
[x, y]-x \otimes y+\theta(a, b) y \otimes x \quad\left(x \in \mathfrak{L}_{a}, y \in \mathfrak{L}_{b}, a, b \in \mathcal{A}\right) .
$$

Then the quotient algebra $U(\mathfrak{L})=\mathcal{T}(\mathfrak{L}) / \mathcal{R}$ together with the natural mapping $\iota: \mathfrak{L} \rightarrow$ $U(\mathfrak{L})$ is the universal enveloping algebra of $\mathfrak{L}$.

For the structure of the universal enveloping algebra $U(\mathfrak{L})$ of $\mathfrak{L}$, the following version of Poincaré-Birkhoff-Witt theorem is well-known.

Theorem 2.2 ( 2], [29], 53])

Let $\mathcal{A}$ be a countable abelian group with a coloring map $\theta: \mathcal{A} \times \mathcal{A} \rightarrow \mathbf{C}^{\times}$and let $\mathfrak{L}=\bigoplus_{a \in \mathcal{A}} \mathfrak{L}_{a}$ be a $\theta$-colored Lie superalgebra. Suppose $X=\left\{x_{\alpha} \mid \alpha \in \Lambda\right\}$ (resp. $Y=\left\{y_{\beta} \mid \beta \in \Omega\right\}$ ) is a homogeneous basis of the subspace $\mathfrak{L}_{0}=\bigoplus_{a \in \mathcal{A}_{0}} \mathfrak{L}_{a}$ (resp. $\left.\mathfrak{L}_{1}=\bigoplus_{b \in \mathcal{A}_{1}} \mathfrak{L}_{b}\right)$. Then the elements of the form

$$
x_{\alpha_{1}} x_{\alpha_{2}} \cdots x_{\alpha_{k}} y_{\beta_{1}} y_{\beta_{2}} \cdots y_{\beta_{l}} \text { with } \alpha_{1} \leq \cdots \leq \alpha_{k}, \beta_{1}<\cdots<\beta_{l}
$$

together with 1 form a basis of the universal enveloping algebra $U(\mathfrak{L})$ of $\mathfrak{L}$. 
Remark. The theory of colored Lie superalgebras can be reduced to the theory of ordinary $\mathbb{Z}_{2}$-graded Lie superalgebras by redefining the superbracket in a suitable way. More precisely, let $\mathfrak{L}=(\mathfrak{L},[]$,$) be a \theta$-colored Lie superalgebra with a coloring map $\theta: \mathcal{A} \times \mathcal{A} \longrightarrow \mathbb{C}^{\times}$and let $X=\left\{x_{i} \mid i=1,2, \cdots\right\}$ be an ordered set of generators of $\mathcal{A}$. We define the bimultiplicative maps $\delta: \mathcal{A} \times \mathcal{A} \longrightarrow \mathbb{C}^{\times}$and $\tau: \mathcal{A} \times \mathcal{A} \longrightarrow \mathbb{C}^{\times}$by

$$
\delta(a, b)=(-1)^{\psi(a) \psi(b)} \theta(b, a)
$$

and

$$
\tau(a, b)=\prod_{i<j} \delta\left(x_{i}, x_{j}\right)^{r_{i} s_{j}},
$$

where $a=\sum r_{i} x_{i}, b=\sum s_{i} x_{i} \in \mathcal{A} \quad\left(r_{i}, s_{i} \in \mathbb{Z}\right)$. We now introduce a new bracket $[,]^{\tau}: \mathfrak{L} \times \mathfrak{L} \longrightarrow \mathfrak{L}$ defined by

$$
[x, y]^{\tau}=\tau(a, b)[x, y]
$$

for $x \in \mathfrak{L}_{a}, y \in \mathfrak{L}_{b}$. Then $\mathfrak{L}^{\tau}=\left(\mathfrak{L},[,]^{\tau}\right)$ becomes an ordinary $\mathbb{Z}_{2}$-graded Lie superalgebra. Furthermore, there is a 1-1 correspondence between the set of $\mathcal{A}$-graded representations of the $\theta$-colored Lie superalgebra $\mathfrak{L}=(\mathfrak{L},[]$,$) and the set of \mathcal{A}$-graded representations of the ordinary Lie superalgebra $\mathfrak{L}^{\tau}=\left(\mathfrak{L},[,]^{\tau}\right)($ cf. [50], [54]).

Let $\mathcal{A}$ be a countable (usually finite) abelian group with a coloring map $\theta$, and let $\Gamma$ be a countable (usually infinite) abelian semigroup such that every element $(\alpha, a) \in$ $\Gamma \times \mathcal{A}$ can be written as a sum of elements in $\Gamma \times \mathcal{A}$ in only finitely many ways. Consider a $(\Gamma \times \mathcal{A})$-graded $\theta$-colored superspace $V=\bigoplus_{(\alpha, a) \in \Gamma \times \mathcal{A}} V_{(\alpha, a)}$ with $\operatorname{dim} V_{(\alpha, a)}<\infty$ for all $(\alpha, a) \in \Gamma \times \mathcal{A}$. Suppose that a group $G$ acts on $V$ preserving the $(\Gamma \times \mathcal{A})$-gradation. We define the generalized character or the graded trace of $V$ for $g \in G$ by

$$
\operatorname{ch}_{g} V=\sum_{(\alpha, a) \in \Gamma \times \mathcal{A}} \operatorname{tr}\left(g \mid V_{(\alpha, a)}\right) e^{(\alpha, a)},
$$

where $e^{(\alpha, a)}$ are the basis elements of the semi-group algebra $\mathbb{C}[\Gamma \times \mathcal{A}]$ with the multiplication given by $e^{(\alpha, a)} e^{(\beta, b)}=e^{(\alpha+\beta, a+b)}$.

On the other hand, we define the supertrace of the homogeneous subspace $V_{(\alpha, a)}$ for $g \in G$ by

$$
\operatorname{str}\left(g \mid V_{(\alpha, a)}\right)=\psi(a) \operatorname{tr}\left(g \mid V_{(\alpha, a)}\right),
$$

and introduce another basis elements of $\mathbb{C}[\Gamma \times \mathcal{A}]$ by setting $E^{(\alpha, a)}=\psi(a) e^{(\alpha, a)}$. Then it is easy to verify that $E^{(\alpha, a)} E^{(\beta, b)}=E^{(\alpha+\beta, a+b)}$. We now define the generalized supercharacter or the graded supertrace of $V$ for $g \in G$ by

$$
\operatorname{sch}_{g} V=\sum_{(\alpha, a) \in \Gamma \times \mathcal{A}} \operatorname{str}\left(g \mid V_{(\alpha, a)}\right) E^{(\alpha, a)} .
$$

Note that $\operatorname{sch}_{g} V$ is obtained from $\operatorname{ch}_{g} V$ by replacing $\operatorname{tr}\left(g \mid V_{(\alpha, a)}\right)=\psi(a) \operatorname{str}\left(g \mid V_{(\alpha, a)}\right)$ and $e^{(\alpha, a)}=\psi(a) E^{(\alpha, a)}$. Since $\psi(a)^{2}=1$, we have $\operatorname{ch}_{g} V=\operatorname{sch}_{g} V$. 
Let $\mathfrak{L}=\bigoplus_{(\alpha, a) \in \Gamma \times \mathcal{A}} \mathfrak{L}_{(\alpha, a)}$ be a $\theta$-colored $(\Gamma \times \mathcal{A})$-graded Lie superalgebra with $\operatorname{dim} \mathfrak{L}_{(\alpha, a)}<\infty$ for all $(\alpha, a) \in \Gamma \times \mathcal{A}$ and suppose that a group $G$ acts on $\mathfrak{L}$ by Lie superalgebra automorphisms preserving the $\Gamma \times \mathcal{A}$-gradation. In this work, using the Euler-Poincaré principle, which is equivalent to the generalized denominator identity, we will derive a closed form formula for the supertraces $\operatorname{str}\left(g \mid \mathfrak{L}_{(\alpha, a)}\right)$ for all $g \in G$, $(\alpha, a) \in \Gamma \times \mathcal{A}$.

Let $\mathbb{C}$ be the trivial one dimensional $\mathfrak{L}$-module. The homology modules $H_{k}(\mathfrak{L})=$ $H_{k}(\mathfrak{L}, \mathbb{C})$ are determined from the following standard complex:

$$
\cdots \rightarrow C_{k}(\mathfrak{L}) \stackrel{d_{k}}{\rightarrow} C_{k-1}(\mathfrak{L}) \stackrel{d_{k-1}}{\rightarrow} \cdots \rightarrow C_{1}(\mathfrak{L}) \stackrel{d_{1}}{\rightarrow} C_{0}(\mathfrak{L}) \rightarrow 0,
$$

where $C_{k}(\mathfrak{L})$ are defined by

$$
C_{k}(\mathfrak{L})=\bigoplus_{p+q=k} \Lambda^{p}\left(\mathfrak{L}_{0}\right) \otimes S^{q}\left(\mathfrak{L}_{1}\right)
$$

and the differentials $d_{k}: C_{k}(\mathfrak{L}) \rightarrow C_{k-1}(\mathfrak{L})$ are given by

$$
\begin{aligned}
& d_{k}\left(\left(x_{1} \wedge \cdots \wedge x_{p}\right) \otimes\left(y_{1} \cdots y_{q}\right)\right) \\
& =\sum_{1 \leq s<t \leq p}(-1)^{s+t}\left(\left[x_{s}, x_{t}\right] \wedge x_{1} \wedge \cdots \wedge \widehat{x_{s}} \wedge \cdots \wedge \widehat{x_{t}} \wedge \cdots \wedge x_{p}\right) \otimes\left(y_{1} \cdots y_{q}\right) \\
& +\sum_{s=1}^{p} \sum_{t=1}^{q}(-1)^{s}\left(x_{1} \wedge \cdots \wedge \widehat{x_{s}} \wedge \cdots \wedge x_{p}\right) \otimes\left(\left[x_{s}, y_{t}\right] y_{1} \cdots \widehat{y_{t}} \cdots y_{q}\right) \\
& -\sum_{1 \leq s<t \leq q}\left(\left[y_{s}, y_{t}\right] \wedge x_{1} \wedge \cdots \wedge x_{p}\right) \otimes\left(y_{1} \cdots \widehat{y_{s}} \cdots \widehat{y_{t}} \cdots y_{q}\right)
\end{aligned}
$$

for $k \geq 2, x_{i} \in \mathfrak{L}_{0}, y_{j} \in \mathfrak{L}_{1}$ and $d_{1}=0$ (cf. [10], [15]). In Section 3, we will give a more detailed treatment of the homology of $\mathfrak{L}$. Since the spaces $C_{k}(\mathfrak{L})$ and the homology modules $H_{k}(\mathfrak{L})$ inherit the $(\Gamma \times \mathcal{A})$-gradation from that of $\mathfrak{L}$, the action of $G$ and the generalized supercharacters of $C_{k}(\mathfrak{L})$ and $H_{k}(\mathfrak{L})$ are well-defined. Hence by the Euler-Poincaré principle, we obtain

$$
\sum_{k=0}^{\infty}(-1)^{k} \operatorname{sch}_{g} C_{k}(\mathfrak{L})=\sum_{k=0}^{\infty}(-1)^{k} \operatorname{sch}_{g} H_{k}(\mathfrak{L}) \text { for all } g \in G .
$$

Let

$$
\begin{aligned}
& C(\mathfrak{L})=\sum_{k=0}^{\infty}(-1)^{k} C_{k}(\mathfrak{L})=\mathbb{C} \ominus L \oplus C_{2}(\mathfrak{L}) \ominus \cdots, \\
& \Lambda\left(\mathfrak{L}_{0}\right)=\sum_{k=0}^{\infty}(-1)^{k} \Lambda^{k}\left(\mathfrak{L}_{0}\right)=\mathbb{C} \ominus \mathfrak{L}_{0} \oplus \Lambda^{2}\left(\mathfrak{L}_{0}\right) \ominus \cdots, \\
& S\left(\mathfrak{L}_{1}\right)=\sum_{k=0}^{\infty}(-1)^{k} S^{k}\left(\mathfrak{L}_{1}\right)=\mathbb{C} \ominus \mathfrak{L}_{1} \oplus S^{2}\left(\mathfrak{L}_{1}\right) \ominus \cdots, \\
& H(\mathfrak{L})=\sum_{k=1}^{\infty}(-1)^{k+1} H_{k}(\mathfrak{L})=H_{1}(\mathfrak{L}) \ominus H_{2}(\mathfrak{L}) \oplus H_{3}(\mathfrak{L}) \ominus \cdots,
\end{aligned}
$$


the alternating direct sum of superspaces. Then it is easy to see that $C(\mathfrak{L})=\Lambda\left(\mathfrak{L}_{0}\right) \otimes$ $S\left(\mathfrak{L}_{1}\right)$

We recall the very basic theory of symmetric functions. Let $x_{1}, \cdots, x_{k}$ be indeterminates and for each $n \in \mathbb{N}$, define

$$
\begin{aligned}
& p_{n}\left(x_{1}, \cdots, x_{k}\right)=\sum_{i=1}^{k} x_{i}^{n}, \\
& h_{n}\left(x_{1}, \cdots, x_{k}\right)=\sum_{i_{1} \leq \cdots \leq i_{n}} x_{i_{1}} \cdots x_{i_{n}}, \\
& e_{n}\left(x_{1}, \cdots, x_{k}\right)=\sum_{i_{1}<\cdots<i_{n}} x_{i_{1}} \cdots x_{i_{n}},
\end{aligned}
$$

which are called the $n$th power sum, the nth completely symmetric function, and the nth elementary symmetric function, respectively. Note that the elementary symmetric function $e_{n}$ is defined only for $n \leq k$. To compute the generalized supercharacters of $\Lambda\left(\mathfrak{L}_{0}\right)$ and $S\left(\mathfrak{L}_{1}\right)$, we recall the following fundamental lemma in the theory of symmetric functions:

Lemma $2.3(46])$

$$
\exp \left(\sum_{r \geq 1} \frac{p_{r}}{r} t^{r}\right)=\sum_{n \geq 0} h_{n} t^{n}=\frac{1}{\sum_{n \geq 0}(-1)^{n} e_{n} t^{n}} .
$$

Let $A$ be an $n \times n$ complex matrix with eigenvalues $x_{1}, \cdots, x_{n}$. By Lemma 2.3, we have

$$
\begin{aligned}
\exp \left(\sum_{r \geq 1} \operatorname{tr}\left(A^{r}\right) \frac{t^{r}}{r}\right) & =\sum_{m \geq 0} \operatorname{tr}\left(A \mid S^{m}\left(\mathbb{C}^{n}\right)\right) t^{m}, \\
\exp \left(-\sum_{r \geq 1} \operatorname{tr}\left(A^{r}\right) \frac{t^{r}}{r}\right) & =\sum_{m \geq 0}(-1)^{m} \operatorname{tr}\left(A \mid \Lambda^{m}\left(\mathbb{C}^{n}\right)\right) t^{m} .
\end{aligned}
$$

Let $t=(t(\alpha, a))_{(\alpha, a) \in \Gamma \times \mathcal{A}}$ be a sequence of nonnegative integers indexed by $\Gamma \times \mathcal{A}$ with only finitely many nonzero terms, and set $|t|=\sum t(\alpha, a)$. Since the $k$ th exterior power $\Lambda^{k}\left(\mathfrak{L}_{0}\right)$ is decomposed as

$$
\Lambda^{k}\left(\mathfrak{L}_{0}\right)=\bigoplus_{|t|=k}\left(\bigotimes_{(\alpha, a) \in \Gamma \times \mathcal{A}_{0}} \Lambda^{t(\alpha, a)}\left(\mathfrak{L}_{(\alpha, a)}\right)\right),
$$

we have

$$
\operatorname{ch}_{g} \Lambda\left(\mathfrak{L}_{0}\right)=\sum_{k=0}^{\infty}(-1)^{k} \operatorname{ch}_{g} \Lambda^{k}\left(\mathfrak{L}_{0}\right)
$$




$$
\begin{aligned}
& =\prod_{(\alpha, a) \in \Gamma \times \mathcal{A}_{0}}\left(\sum_{m=0}^{\operatorname{dim} \mathfrak{L}_{(\alpha, a)}}(-1)^{m} \operatorname{tr}\left(g \mid \Lambda^{m}\left(\mathfrak{L}_{(\alpha, a)}\right)\right) e^{m(\alpha, a)}\right) \\
& =\prod_{(\alpha, a) \in \Gamma \times \mathcal{A}_{0}}\left(\sum_{m \geq 0}(-1)^{m} \operatorname{tr}\left(g \mid \Lambda^{m}\left(\mathfrak{L}_{(\alpha, a)}\right)\right) e^{m(\alpha, a)}\right) \\
& =\prod_{(\alpha, a) \in \Gamma \times \mathcal{A}_{0}} \exp \left(-\sum_{r=1}^{\infty} \frac{1}{r} \operatorname{tr}\left(g^{r} \mid \mathfrak{L}_{(\alpha, a)}\right) e^{r(\alpha, a)}\right)
\end{aligned}
$$

for all $g \in G$. Similarly, we have

$$
\begin{aligned}
\operatorname{ch}_{g} S\left(\mathfrak{L}_{1}\right) & =\sum_{k=0}^{\infty}(-1)^{k} \operatorname{ch}_{g} S^{k}\left(\mathfrak{L}_{1}\right) \\
& =\prod_{(\alpha, a) \in \Gamma \times \mathcal{A}_{1}}\left(\sum_{m \geq 0}(-1)^{m} \operatorname{tr}\left(g \mid S^{m}\left(\mathfrak{L}_{(\beta, b)}\right)\right) e^{m(\beta, b)}\right) \\
& =\prod_{(\alpha, a) \in \Gamma \times \mathcal{A}_{1}}\left(\sum_{m \geq 0} \operatorname{tr}\left(g \mid S^{m}\left(\mathfrak{L}_{(\beta, b)}\right)\right)\left(-e^{(\beta, b)}\right)^{m}\right) \\
& =\prod_{(\alpha, a) \in \Gamma \times \mathcal{A}_{1}} \exp \left(\sum_{r=1}^{\infty} \frac{(-1)^{r}}{r} \operatorname{tr}\left(g^{r} \mid \mathfrak{L}_{(\beta, b)}\right) e^{r(\beta, b)}\right) .
\end{aligned}
$$

It follows that

$$
\begin{aligned}
& \operatorname{ch}_{g} C(\mathfrak{L})=\sum_{k=0}^{\infty}(-1)^{k} \operatorname{ch}_{g} C_{k}(\mathfrak{L})=\operatorname{ch}_{g} \Lambda\left(\mathfrak{L}_{0}\right) \cdot \operatorname{ch}_{g} S\left(\mathfrak{L}_{1}\right) \\
& =\prod_{(\alpha, a) \in \Gamma \times \mathcal{A}_{0}} \exp \left(-\sum_{r=1}^{\infty} \frac{1}{r} \operatorname{tr}\left(g^{r} \mid \mathfrak{L}_{(\alpha, a)}\right) e^{r(\alpha, a)}\right) \\
& \times \prod_{(\beta, b) \in \Gamma \times \mathcal{A}_{1}} \exp \left(\sum_{r=1}^{\infty} \frac{(-1)^{r}}{r} \operatorname{tr}\left(g^{r} \mid \mathfrak{L}_{(\beta, b)}\right) e^{r(\beta, b)}\right) .
\end{aligned}
$$

Replacing $E^{(\alpha, a)}=\psi(a) e^{(\alpha, a)}$ and $\operatorname{str}\left(g \mid \mathfrak{L}_{(\alpha, a)}\right)=\psi(a) \operatorname{tr}\left(g \mid \mathfrak{L}_{(\alpha, a)}\right)$, the above identity yields

$$
\operatorname{sch}_{g} C(\mathfrak{L})=\prod_{(\alpha, a) \in \Gamma \times \mathcal{A}} \exp \left(-\sum_{r=1}^{\infty} \frac{1}{r} \operatorname{str}\left(g^{r} \mid \mathfrak{L}_{(\alpha, a)}\right) E^{r(\alpha, a)}\right) .
$$

Hence, by the Euler-Poincaré principle, we obtain the generalized denominator identity for the $\theta$-colored graded Lie superalgebra $\mathfrak{L}=\bigoplus_{(\alpha, a) \in \Gamma \times \mathcal{A}} \mathfrak{L}_{(\alpha, a)}$ :

$$
\prod_{(\alpha, a) \in \Gamma \times \mathcal{A}} \exp \left(-\sum_{r=1}^{\infty} \frac{1}{r} \operatorname{str}\left(g^{r} \mid \mathfrak{L}_{(\alpha, a)}\right) E^{r(\alpha, a)}\right)=1-\operatorname{sch}_{g} H(\mathfrak{L}) .
$$


When $g=1$, the identity element of $G$, we get the denominator identity for the Lie superalgebra $\mathfrak{L}(40)$ :

$$
\prod_{\Gamma \times \mathcal{A}}\left(1-E^{(\alpha, a)}\right)^{\operatorname{sdim} \mathfrak{L}_{(\alpha, a)}}=1-\operatorname{sch} H(\mathfrak{L}) .
$$

Let $P(\Gamma \times \mathcal{A})=\left\{(\alpha, a) \in \Gamma \times \mathcal{A} \mid \operatorname{str}\left(g \mid H(\mathfrak{L})_{(\alpha, a)}\right) \neq 0\right\}$ and $\left\{\left(\alpha_{i}, a_{j}\right) \mid i, j=\right.$ $1,2,3, \cdots\}$ be an enumeration of $P(\Gamma \times \mathcal{A})$. For $(\tau, b) \in \Gamma \times \mathcal{A}$, set

$$
T(\tau, b)=\left\{s=\left(s_{i j}\right) \mid s_{i j} \geq 0, \quad \sum_{i, j} s_{i j}\left(\alpha_{i}, a_{j}\right)=(\tau, b)\right\},
$$

the set of all partitions of $(\tau, b)$ into a sum of $\left(\alpha_{i}, a_{j}\right)$ 's, and define

$$
W_{g}(\tau, b)=\sum_{s \in T(\tau, b)} \frac{(|s|-1) !}{s !} \prod_{i, j} \operatorname{str}\left(g \mid H(\mathfrak{L})_{\left(\alpha_{i}, a_{j}\right)}\right)^{s_{i j}}
$$

We will call $W_{g}(\tau, b)$ the Witt partition function for $g \in G$. In the next theorem, using the generalized denominator identity (2.14), we derive a closed form formula for the supertraces $\operatorname{str}\left(g \mid \mathfrak{L}_{(\alpha, a)}\right)((\alpha, a) \in \Gamma \times \mathcal{A})$ in terms of the Witt partition functions.

Theorem 2.4 For $g \in G$ and $(\alpha, a) \in \Gamma \times \mathcal{A}$, we have

$$
\operatorname{str}\left(g \mid \mathfrak{L}_{(\alpha, a)}\right)=\sum_{\substack{d>0 \\(\alpha, a)=d(\tau, b)}} \frac{1}{d} \mu(d) W_{g^{d}}(\tau, b) .
$$

Proof. By the generalized denominator idenity (2.14), we have

$$
\begin{array}{r}
\exp \left(\sum_{(\alpha, a) \in \Gamma \times \mathcal{A}} \sum_{r=1}^{\infty} \frac{1}{r} \operatorname{str}\left(g^{r} \mid \mathfrak{L}_{(\alpha, a)}\right) E^{r(\alpha, a)}\right) \\
=\frac{1}{1-\sum_{i, j=1}^{\infty} \operatorname{str}\left(g \mid H(\mathfrak{L})_{\left(\alpha_{i}, a_{j}\right)}\right) E^{\left(\alpha_{i}, a_{j}\right)}} .
\end{array}
$$

Taking the logarithm and using the formal power series $\log (1-x)=-\sum_{k=1}^{\infty} \frac{1}{k} x^{k}$, we obtain

$$
\begin{aligned}
& \sum_{(\alpha, a) \in \Gamma \times \mathcal{A}} \sum_{r=1}^{\infty} \frac{1}{r} \operatorname{str}\left(g^{r} \mid \mathfrak{L}_{(\alpha, a)}\right) E^{r(\alpha, a)} \\
& =-\log \left(1-\sum_{i, j=1}^{\infty} \operatorname{str}\left(g \mid H(\mathfrak{L})_{\left(\alpha_{i}, a_{j}\right)}\right) E^{\left(\alpha_{i}, a_{j}\right)}\right)^{-1} \\
& =\sum_{m=1}^{\infty} \frac{1}{m}\left(\sum_{i, j=1}^{\infty} \operatorname{str}\left(g \mid H(\mathfrak{L})_{\left(\alpha_{i}, a_{j}\right)}\right) E^{\left(\alpha_{i}, a_{j}\right)}\right)^{m}
\end{aligned}
$$




$$
\begin{aligned}
& =\sum_{m=1}^{\infty} \frac{1}{m} \sum_{\substack{s=\left(s_{i j}\right) \\
\sum s_{i j}=m}} \frac{\left(\sum s_{i j}\right) !}{\prod s_{i j} !} \prod \operatorname{str}\left(g \mid H(\mathfrak{L})_{\left(\alpha_{i}, a_{j}\right)}\right)^{s_{i j}} E^{\sum s_{i j}\left(\alpha_{i}, a_{j}\right)} \\
& =\sum_{(\tau, b) \in \Gamma \times \mathcal{A}}\left(\sum_{s \in T(\tau, b)} \frac{\left(\sum s_{i j}-1\right) !}{\prod s_{i j} !} \prod \operatorname{str}\left(g \mid H(\mathfrak{L})_{\left(\alpha_{i}, a_{j}\right)}\right)^{s_{i j}}\right) E^{(\tau, b)} \\
& =\sum_{(\tau, b) \in \Gamma \times \mathcal{A}} W_{g}(\tau, b) E^{(\tau, b)} .
\end{aligned}
$$

It follows that

$$
W_{g}(\tau, b)=\sum_{\substack{k>0 \\(\tau, b)=k(\alpha, a)}} \frac{1}{k} \operatorname{str}\left(g^{k} \mid \mathfrak{L}_{(\alpha, a)}\right) .
$$

Hence, by Möbius inversion, we obtain

$$
\operatorname{str}\left(g \mid \mathfrak{L}_{(\alpha, a)}\right)=\sum_{\substack{d>0 \\ d(\tau, b)=(\alpha, a)}} \frac{1}{d} \mu(d) W_{g^{d}}(\tau, b) .
$$

Remark. Note that our supertrace formula is a generalization of the trace formula for graded Lie algebras obtained in [32]. Also, when $g=1$, our supertrace formula yields the superdimension formula for homogeneous subspaces of graded Lie superalgebras (cf. 40]).

\section{Homology of Lie Superalgebras}

The homology group of a Lie algebra is defined as the torsion group of its universal enveloping algebra and characterized by Koszul's complex. Similarly, we define the homology group of a graded Lie superalgebra as the torsion group of its universal enveloping algebra viewed as a supplmented algebra (cf. [10], 15]). In this section, we will show that there is a super-analogue of Koszul's complex for a graded Lie superalgebra and characterize its homology group. Our argument follows the framework given in 110 and $[15$.

Let $\mathfrak{L}=\mathfrak{L}_{0} \oplus \mathfrak{L}_{1}$ be a Lie superalgebra and $U=U(\mathfrak{L})$ be its universal enveloping algebra. For each $k \geq 0$, define

$$
C_{k}=C_{k}(\mathfrak{L})=\bigoplus_{p+q=k} \Lambda^{p}\left(\mathfrak{L}_{0}\right) \otimes S^{q}\left(\mathfrak{L}_{1}\right)
$$


Consider the following chain complex $\left(M_{k}, \partial_{k}^{ \pm}\right)(k \geq-1)$, where

$$
M_{k}= \begin{cases}U \otimes_{\mathbb{C}} C_{k}(\mathfrak{L}) & \text { if } k \geq 0, \\ \mathbb{C} & \text { if } k=-1,\end{cases}
$$

and the differentials $\partial_{k}: M_{k} \rightarrow M_{k-1}$ are given by

$$
\begin{aligned}
& \partial_{k}\left(u \otimes\left(x_{1} \wedge \cdots \wedge x_{p}\right) \otimes\left(y_{1} \cdots y_{q}\right)\right) \\
& =\sum_{1 \leq s<t \leq p}(-1)^{s+t} u \otimes\left(\left[x_{s}, x_{t}\right] \wedge x_{1} \wedge \cdots \wedge \widehat{x_{s}} \wedge \cdots \wedge \widehat{x_{t}} \wedge \cdots \wedge x_{p}\right) \otimes\left(y_{1} \cdots y_{q}\right) \\
& +\sum_{s=1}^{p} \sum_{t=1}^{q}(-1)^{s} u \otimes\left(x_{1} \wedge \cdots \wedge \widehat{x_{s}} \wedge \cdots \wedge x_{p}\right) \otimes\left(\left[x_{s}, y_{t}\right] y_{1} \cdots \widehat{y_{t}} \cdots y_{q}\right) \\
& -\sum_{1 \leq s<t \leq q} u \otimes\left(\left[y_{s}, y_{t}\right] \wedge x_{1} \wedge \cdots \wedge x_{p}\right) \otimes\left(y_{1} \cdots \widehat{y}_{s} \cdots \widehat{y}_{t} \cdots y_{q}\right) \\
& +\sum_{s=1}^{p}(-1)^{s+1}\left(u \cdot x_{s}\right) \otimes\left(x_{1} \wedge \cdots \wedge \widehat{x_{s}} \wedge \cdots \wedge x_{p}\right) \otimes\left(y_{1} \cdots y_{q}\right) \\
& +(-1)^{p} \sum_{t=1}^{q}\left(u \cdot y_{t}\right) \otimes\left(x_{1} \wedge \cdots \wedge x_{p}\right) \otimes\left(y_{1} \cdots \widehat{y}_{t} \cdots y_{q}\right)
\end{aligned}
$$

for $k \geq 1, \partial_{0}$ is an augmentation map extracting the constant term, and $\partial_{-1}=0$. Then it is easy to verify that $\partial_{k-1} \circ \partial_{k}=0$.

Proposition 3.1 The chain complex $M=\left(M_{k}, \partial_{k}\right)$ is a free resolution of the trivial 1-dimensional left $U$-module $\mathbb{C}$.

Proof. Case 1. Suppose first that $\mathfrak{L}$ is abelian. Then, by the Poincaré-Birkhoff-Witt Theorem, we have

$$
U(\mathfrak{L}) \simeq S\left(\mathfrak{L}_{0}\right) \otimes \Lambda\left(\mathfrak{L}_{1}\right)
$$

as a $\mathbb{C}$-vector space, and the differential maps are simplified to

$$
\begin{aligned}
& \partial_{k}\left(u \otimes\left(x_{1} \wedge \cdots \wedge x_{p}\right) \otimes\left(y_{1} \cdots y_{q}\right)\right. \\
& =\sum_{s=1}^{p}(-1)^{s+1}\left(u \cdot x_{s}\right) \otimes\left(x_{1} \wedge \cdots \wedge \widehat{x_{s}} \wedge \cdots \wedge x_{p}\right) \otimes\left(y_{1} \cdots y_{q}\right) \\
& +(-1)^{p} \sum_{t=1}^{q}\left(u \cdot y_{t}\right) \otimes\left(x_{1} \wedge \cdots \wedge x_{p}\right) \otimes\left(y_{1} \cdots \widehat{y_{t}} \cdots y_{q}\right) .
\end{aligned}
$$

We define the homotopy map $D: M_{k} \rightarrow M_{k+1}$ by

$$
\begin{aligned}
& D\left(\left(u_{1} \cdots u_{s} v_{1} \cdots v_{t}\right) \otimes\left(x_{1} \wedge \cdots \wedge x_{p}\right) \otimes\left(y_{1} \cdots y_{q}\right)\right) \\
& =\sum_{i=1}^{s}\left(u_{1} \cdots \widehat{u}_{i} \cdots u_{s} v_{1} \cdots v_{t}\right) \otimes\left(u_{i} \wedge x_{1} \wedge \cdots \wedge x_{p}\right) \otimes\left(y_{1} \cdots y_{q}\right) \\
& =\sum_{j=1}^{t}(-1)^{p+t-j}\left(u_{1} \cdots u_{s} v_{1} \cdots \widehat{v}_{j} \cdots v_{t}\right) \otimes\left(x_{1} \wedge \cdots \wedge x_{p}\right) \otimes\left(v_{j} y_{1} \cdots y_{q}\right),
\end{aligned}
$$


where $u_{i} \in \mathfrak{L}_{0}$ and $v_{j} \in \mathfrak{L}_{1}$. Then it is easy to verify that

$$
D \partial+\partial D=(p+q+s+t) 1_{M}
$$

which implies that our complex $M=\left(M_{k}, \partial_{k}\right)$ is exact in this case.

Case 2. Let $\mathfrak{L}$ be any Lie superalgebra. Recall the construction of the universal enveloping algebra of $\mathfrak{L}$. Put $U^{n}(\mathfrak{L})=\pi\left(\bigoplus_{k=0}^{n} \mathfrak{L}^{\otimes k}\right)$, where $\pi: \mathcal{T}(\mathfrak{L}) \rightarrow U(\mathfrak{L})$ is the canonical projection map and $X_{n}^{(p)}=U^{p-n}(\mathfrak{L}) \otimes C_{n}(\mathfrak{L})$ for $0 \leq n \leq p$.

Consider the complex

$$
0 \longrightarrow X_{p}^{(p)} \stackrel{\partial_{p}}{\longrightarrow} \cdots \stackrel{\partial_{2}}{\longrightarrow} X_{1}^{(p)} \stackrel{\partial_{1}}{\longrightarrow} X_{0}^{(p)} \stackrel{\epsilon}{\longrightarrow} \mathbb{C} \longrightarrow 0 .
$$

We will use the induction on $p$ to prove the exactness of (3.4), which would imply the exactness of our original sequence.

Note that the exactness of (3.4) is equivalent to that of the following sequence:

$$
0 \longrightarrow X_{p}^{(p)} \stackrel{\partial_{p}}{\longrightarrow} \cdots \stackrel{\partial_{2}}{\longrightarrow} X_{1}^{(p)} \stackrel{\partial_{1}}{\longrightarrow} X_{0}^{(p)} / \mathbb{C} \longrightarrow 0 .
$$

For $p=0,1$, our assertion is clear. Suppose (3.5) is exact for $p-1$. Consider the diagram

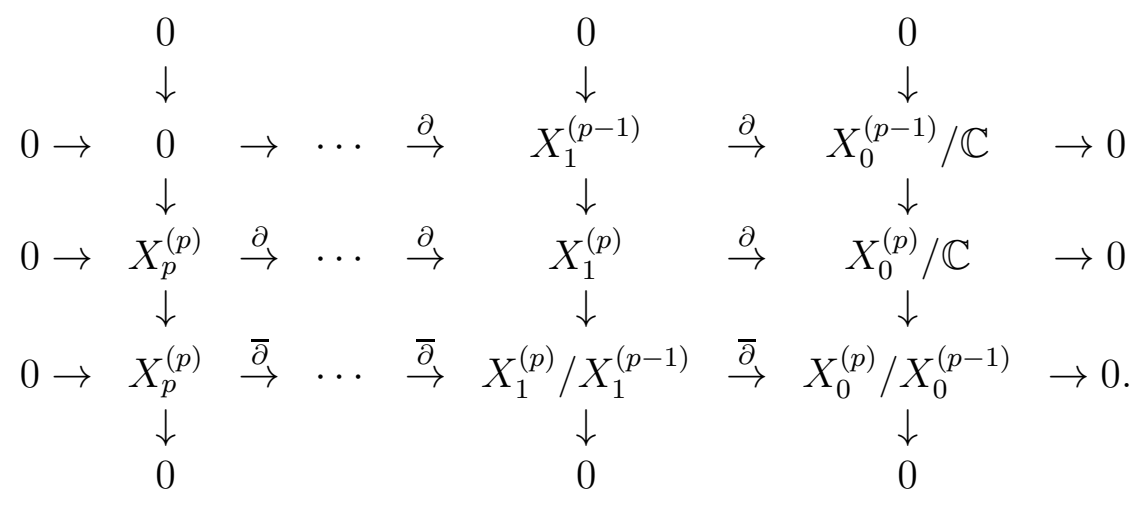

Note that

$$
X_{n}^{(p)} / X_{n}^{(p-1)} \simeq\left(U^{p-n}(\mathfrak{L}) / U^{p-n-1}(\mathfrak{L})\right) \otimes C_{n}(\mathfrak{L}) \quad(0 \leq n \leq p-1)
$$

as a $\mathbb{C}$-vector space, and that, by the Poincaré-Birkhoff-Witt Theorem,

$$
U^{p-n}(\mathfrak{L}) / U^{p-n-1}(\mathfrak{L}) \simeq \bigoplus_{k+l=p-n} S^{k}\left(\mathfrak{L}_{0}\right) \otimes \Lambda^{l}\left(\mathfrak{L}_{1}\right)
$$

by the canonical homomorphism and $X_{p}^{(p)} \simeq \mathbb{C} \otimes C_{p}(\mathfrak{L})$. Finally, the differentials in bottom row are given by

$$
\begin{aligned}
& \overline{\partial_{n}}\left(u \otimes\left(x_{1} \wedge \cdots \wedge x_{p}\right) \otimes\left(y_{1} \cdots y_{q}\right)+X_{n}^{(p-1)}\right) \\
& =\sum_{s=1}^{p}(-1)^{s+1}\left(u \cdot x_{s}\right) \otimes\left(x_{1} \wedge \cdots \wedge \widehat{x_{s}} \wedge \cdots \wedge x_{p}\right) \otimes\left(y_{1} \cdots y_{q}\right) \\
& +(-1)^{p} \sum_{t=1}^{q}\left(u \cdot y_{t}\right) \otimes\left(x_{1} \wedge \cdots \wedge x_{p}\right) \otimes\left(y_{1} \cdots \widehat{y_{t}} \cdots y_{q}\right)+X_{n-1}^{(p-1)} .
\end{aligned}
$$


Thus the bottom row can be viewed as a subcomplex of the complex considered in Case 1 , and hence it is exact. Therefore, the middle row is exact, which completes our induction argument.

Since $C(\mathfrak{L})$ is a $\mathbb{C}$-vector space, $U \otimes C(\mathfrak{L})$ is the direct sum of free $U$-modules. Hence, $U \otimes C(\mathfrak{L})$ is a free resolution of the 1 -dimensional trivial left $U$-module $\mathbb{C}$.

Let $V$ be a right $U$-module. The homology module $H_{k}(\mathfrak{L}, V)$ is defined to be

$$
H_{k}(\mathfrak{L}, V)=\operatorname{Tor}_{k}^{U}(V, \mathbb{C})=H_{k}\left(V \otimes_{U} M\right)
$$

where $M=\left(M_{k}, \partial_{k}\right)$ is the free resolution of the 1-dimensional trivial left $U$-module $\mathbb{C}$ given by Proposition 3.1. Since we have

$$
V \otimes_{U} M \cong V \otimes_{U} U \otimes_{\mathbb{C}} C_{k}(\mathfrak{L}) \cong V \otimes_{\mathbb{C}} C_{k}(\mathfrak{L}) \quad(k \geq 0)
$$

and

$$
V \otimes_{U} \mathbb{C} \cong V
$$

as $\mathbb{C}$-vector spaces, we obtain the following standard complex for the homology of Lie superalgebras:

$$
\cdots \longrightarrow V \otimes_{\mathbb{C}} C_{k}(\mathfrak{L}) \stackrel{d_{k}}{\longrightarrow} V \otimes_{\mathbb{C}} C_{k-1}(\mathfrak{L}) \stackrel{d_{k-1}}{\longrightarrow} \cdots \stackrel{d_{1}}{\longrightarrow} V \otimes_{\mathbb{C}} C_{0}(\mathfrak{L}) \cong V \longrightarrow 0,
$$

where the differentials $d_{k}=1_{V} \otimes_{U} \partial_{k}: V \otimes_{\mathbb{C}} C_{k}(\mathfrak{L}) \longrightarrow V \otimes_{\mathbb{C}} C_{k-1}(\mathfrak{L})$ are given by

$$
\begin{aligned}
& d_{k}\left(v \otimes\left(x_{1} \wedge \cdots \wedge x_{p}\right) \otimes\left(y_{1} \cdots y_{q}\right)\right) \\
& =\sum_{1 \leq s<t \leq p}(-1)^{s+t} v \otimes\left(\left[x_{s}, x_{t}\right] \wedge x_{1} \wedge \cdots \wedge \widehat{x_{s}} \wedge \cdots \wedge \widehat{x}_{t} \wedge \cdots \wedge x_{p}\right) \otimes\left(y_{1} \cdots y_{q}\right) \\
& +\sum_{s=1}^{p} \sum_{t=1}^{q}(-1)^{s} v \otimes\left(x_{1} \wedge \cdots \wedge \widehat{x_{s}} \wedge \cdots \wedge x_{p}\right) \otimes\left(\left[x_{s}, y_{t}\right] y_{1} \cdots \widehat{y_{t}} \cdots y_{q}\right) \\
& -\sum_{1 \leq s<t \leq q} v \otimes\left(\left[y_{s}, y_{t}\right] \wedge x_{1} \wedge \cdots \wedge x_{p}\right) \otimes\left(y_{1} \cdots \widehat{y}_{s} \cdots \widehat{y}_{t} \cdots y_{q}\right) \\
& +\sum_{s=1}^{p}(-1)^{s+1}\left(v \cdot x_{s}\right) \otimes\left(x_{1} \wedge \cdots \wedge \widehat{x_{s}} \wedge \cdots \wedge x_{p}\right) \otimes\left(y_{1} \cdots y_{q}\right) \\
& +(-1)^{p} \sum_{t=1}^{q}\left(v \cdot y_{t}\right) \otimes\left(x_{1} \wedge \cdots \wedge x_{p}\right) \otimes\left(y_{1} \cdots \widehat{y_{t}} \cdots y_{q}\right)
\end{aligned}
$$

for $v \in V, x_{i} \in \mathfrak{L}_{0}, y_{j} \in \mathfrak{L}_{1}$. In particular, when $V=\mathbb{C}$ is the trivial 1-dimensional right $U$-module, the standard complex (3.8) is reduced to the complex (2.8) given in Section 2 .

As a corollary of Proposition 3.1, we obtain: 
Corollary 3.2 Let $\mathcal{A}$ be a countable abelian group with a coloring map $\theta$ and $V=$ $\oplus_{a \in \mathcal{A}} V_{a}$ be a $\theta$-colored superspace. Let $\mathfrak{L}$ be the free Lie superalgebra generated by $V$. Then we have

$$
H_{k}(\mathfrak{L})= \begin{cases}V & \text { if } k=1, \\ 0 & \text { if } k \geq 2 .\end{cases}
$$

Proof. Note that the universal enveloping algebra of $\mathfrak{L}$ is isomorphic to the tensor algebra $\mathcal{T}(V)$ generated by $V$. Consider the following free resolution of the 1-dimensional trivial left $\mathcal{T}(V)$-module $\mathbb{C}$ :

$$
0 \rightarrow \mathcal{T}(V) \otimes V \stackrel{\varphi}{\rightarrow} \mathcal{T}(V) \stackrel{\psi}{\rightarrow} \mathbb{C} \simeq \mathcal{T}(V) / \mathcal{T}(V) \otimes V \rightarrow 0,
$$

where $\varphi$ is the natural inclusion map $(=$ multiplication map) and $\psi$ is the augmentation map.

By tensoring with trivial right $\mathcal{T}(V)$-module $\mathbb{C}$, we get

$$
0 \rightarrow \mathbb{C} \otimes_{\mathcal{T}(V)} \mathcal{T}(V) \otimes V \stackrel{1 \otimes \varphi}{\rightarrow} \mathbb{C} \otimes_{\mathcal{T}(V)} \mathcal{T}(V) \stackrel{1 \otimes \psi}{\rightarrow} \mathbb{C} \otimes \mathbb{C} \rightarrow 0 .
$$

Since $1 \otimes \varphi$ is a zero map, $H_{1}(\mathcal{L})=\operatorname{Ker}(1 \otimes \varphi)=\mathbb{C} \otimes_{\mathcal{T}(V)} \mathcal{T}(V) \otimes V \simeq V$, and $H_{k}(\mathcal{L})=0$ for $k \geq 2$.

\section{Free Lie Algebras and Free Lie Superalgebras}

Let $\mathcal{A}$ be a countable abelian group with a coloring map $\theta$ and $\Gamma$ be a countable abelian semigroup satisfying the finiteness condition given in Section 2. Let $V=$ $\bigoplus_{(\alpha, a) \in \Gamma \times \mathcal{A}} V_{(\alpha, a)}$ be a $(\Gamma \times \mathcal{A})$-graded $\theta$-colored superspace with finite dimensional homogeneous subspaces, and let $\mathfrak{L}=\bigoplus_{(\alpha, a) \in \Gamma \times \mathcal{A}} \mathfrak{L}_{(\alpha, a)}$ be the free Lie superalgebra generated by $V$. Suppose that a group $G$ acts on $V$ preserving the $(\Gamma \times \mathcal{A})$-gradation. Then the action of $G$ on $V$ can be extended to $\mathfrak{L}$ by Lie superalgebra automorphisms.

As we have seen in Section 3, we have $H_{1}(\mathfrak{L})=V$ and $H_{k}(\mathfrak{L})=0$ for $k \geq 2$. Hence, for any $g \in G$, the generalized denominator identity for the free Lie superalgebra $\mathfrak{L}$ is equal to

$$
\begin{aligned}
\prod_{(\alpha, a) \in \Gamma \times \mathcal{A}} & \exp \left(-\sum_{k=1}^{\infty} \frac{1}{k} \operatorname{str}\left(g^{k} \mid \mathfrak{L}_{(\alpha, a)}\right) E^{k(\alpha, a)}\right) \\
& =1-\operatorname{sch}_{g} V=1-\sum_{(\alpha, a) \in \Gamma \times \mathcal{A}} \operatorname{str}\left(g \mid V_{(\alpha, a)}\right) E^{(\alpha, a)} .
\end{aligned}
$$

For any $g \in G$, let $P(V, \Gamma \times \mathcal{A})=\left\{(\alpha, a) \in \Gamma \times \mathcal{A} \mid \operatorname{str}\left(g \mid V_{(\alpha, a)}\right) \neq 0\right\}$ and let $\left\{\left(\alpha_{i}, a_{j}\right) \mid i, j=1,2,3, \cdots\right\}$ be an enumeration of the set $P(V, \Gamma \times \mathcal{A})$. For each 
$(\tau, b) \in \Gamma \times \mathcal{A}$, we denote by $T(\tau, b)$ the set of all paritions of $(\tau, b)$ into a sum of $\left(\alpha_{i}, a_{j}\right)$ 's as defined in (2.15), and let $W_{g}(\tau, b)$ be the Witt partition function as defined in (2.16). Then our supertrace formula (2.17) yields

$$
\operatorname{str}\left(g \mid \mathfrak{L}_{(\alpha, a)}\right)=\sum_{\substack{d>0 \\(\alpha, a)=d(\tau, b)}} \frac{1}{d} \mu(d) \sum_{s \in T(\tau, b)} \frac{(|s|-1) !}{s !} \prod_{i, j} \operatorname{str}\left(g^{d} \mid V_{\left(\alpha_{i}, a_{j}\right)}\right)^{s_{i j}} .
$$

Remark. The supertrace formula (4.2) is a generalization of the superdimension formula for the free Lie superalgebras given in [39] and [40].

Example 4.1 In this example, we will consider the simplest application of our supertrace formula to free Lie superalgebras. Consider the superspace $V=V_{0} \oplus V_{1}$ with $\operatorname{dim} V_{0}=r$ and $\operatorname{dim} V_{1}=s$ for some $r, s \in \mathbb{Z}_{>0}$, and let $\mathfrak{L}$ be the free Lie superalgebra generated by $V$. By setting $\operatorname{deg} v=1$ for all $v \in V$, the free Lie superalgebra $\mathfrak{L}$ has a $\mathbb{Z}_{>0}$-gradation $\mathfrak{L}=\bigoplus_{n=1}^{\infty} \mathfrak{L}_{n}$ induced by $V$. Let $G=G L\left(V_{0}\right) \times G L\left(V_{1}\right) \cong$ $G L(r) \times G L(s)$. For $(g, h) \in G$, we denote by $t_{g}=\operatorname{tr}\left(g \mid V_{0}\right)$ and $t_{h}=\operatorname{tr}\left(h \mid V_{1}\right)$. Since $P\left(V, \mathbb{Z}_{>0}\right)=\{1\}$ and $\operatorname{str}((g, h) \mid V)=\operatorname{tr}\left(g \mid V_{0}\right)-\operatorname{tr}\left(h \mid V_{1}\right)=t_{g}-t_{h}$ for all $(g, h) \in G$, the Witt partition function $W_{(g, h)}(n)$ is given by

$$
W_{(g, h)}(n)=\frac{(n-1) !}{n !}\left(t_{g}-t_{h}\right)^{n}=\frac{1}{n}\left(t_{g}-t_{h}\right)^{n} .
$$

Hence, by the supertrace formula (4.2), we have

$$
\operatorname{str}\left((g, h) \mid \mathfrak{L}_{n}\right)=\frac{1}{n} \sum_{d \mid n} \mu(d)\left(t_{g}-t_{h}\right)^{\frac{n}{d}} .
$$

Next, we will discuss the relation of the free Lie algebra and the free Lie superalgebra generated by the same vector space $V=\bigoplus_{(\alpha, a) \in \Gamma \times \mathcal{A}} V_{(\alpha, a)}$ on which a group $G$ acts preserving the $(\Gamma \times \mathcal{A})$-gradation. By neglecting the coloring map $\theta$ on $\mathcal{A}$, consider $V$ as a $(\Gamma \times \mathcal{A})$-graded vector space, and let $L=\oplus_{(\alpha, a) \in \Gamma \times \mathcal{A}} L_{(\alpha, a)}$ be the free Lie algebra generated by $V$. Then the action of $G$ on $V$ can be extended to $L$ by Lie algebra automorphisms. Hence, for any $g \in G$, the generalized ednominator identity for $L$ is equal to

$$
\begin{aligned}
\prod_{(\alpha, a) \in \Gamma \times \mathcal{A}} \exp \left(-\sum_{k=1}^{\infty} \frac{1}{k} \operatorname{tr}\left(g^{k} \mid L_{(\alpha, a)}\right) e^{k(\alpha, a)}\right) \\
=1-\operatorname{ch}_{g} V=1-\sum_{(\alpha, a) \in \Gamma \times \mathcal{A}} \operatorname{tr}\left(g \mid V_{(\alpha, a)}\right) e^{(\alpha, a)} .
\end{aligned}
$$

Since $E^{(\alpha, a)}=\psi(a) e^{(\alpha, a)}$ and $\operatorname{str}\left(g \mid V_{(\alpha, a)}\right)=\psi(a) \operatorname{tr}\left(g \mid V_{(\alpha, a)}\right)$ for all $(\alpha, a) \in \Gamma \times \mathcal{A}$, the right-hand sides of (4.1) and (4.4) are the same. Moreover, note that, for an $n \times n$ 
complex matrix $A$ with eigenvalues $x_{1}, \cdots, x_{n}$, Lemma 2.1 implies

$$
\begin{aligned}
& \exp \left(\sum_{k=1}^{\infty} \frac{1}{k} \operatorname{tr}\left(A^{k}\right) t^{k}\right) \exp \left(\sum_{k=1}^{\infty} \frac{1}{k}(-1)^{k} \operatorname{tr}\left(A^{k}\right) t^{k}\right) \\
& =\prod \frac{1}{1-x_{i} t} \prod \frac{1}{1+x_{i} t}=\prod \frac{1}{1-x_{i}^{2} t^{2}} \\
& =\sum_{n=0}^{\infty} h_{n}\left(x_{1}^{2}, \cdots, x_{n}^{2}\right) t^{2 n}=\exp \left(\sum_{k=1}^{\infty} \frac{1}{k} \operatorname{tr}\left(A^{2 k}\right) t^{2 k}\right) .
\end{aligned}
$$

Therefore, the left-hand side of (4.4) is equal to

$$
\begin{aligned}
\prod_{(\alpha, a) \in \Gamma \times \mathcal{A}_{0}} & \exp \left(-\sum_{k=1}^{\infty} \frac{1}{k} \operatorname{tr}\left(g^{k} \mid L_{(\alpha, a)}\right) e^{k(\alpha, a)}\right) \prod_{(\beta, b) \in \Gamma \times \mathcal{A}_{1}} \exp \left(-\sum_{k=1}^{\infty} \frac{1}{k} \operatorname{tr}\left(g^{k} \mid L_{(\beta, b)}\right) e^{k(\beta, b)}\right) \\
= & \prod_{(\alpha, a) \in \Gamma \times \mathcal{A}_{0}} \exp \left(-\sum_{k=1}^{\infty} \frac{1}{k} \operatorname{tr}\left(g^{k} \mid L_{(\alpha, a)}\right) E^{k(\alpha, a)}\right) \\
& \times \prod_{(\beta, b) \in \Gamma \times \mathcal{A}_{1}} \exp \left(-\sum_{k=1}^{\infty} \frac{(-1)^{k}}{k} \operatorname{tr}\left(g^{k} \mid L_{(\beta, b)}\right) E^{k(\beta, b)}\right) \\
= & \prod_{(\alpha, a) \in \Gamma \times \mathcal{A}_{0}} \exp \left(-\sum_{k=1}^{\infty} \frac{1}{k} \operatorname{tr}\left(g^{k} \mid L_{(\alpha, a)}\right) E^{k(\alpha, a)}\right) \\
& \times \prod_{(\beta, b) \in \Gamma \times \mathcal{A}_{1}} \exp \left(\sum_{k=1}^{\infty} \frac{1}{k} \operatorname{tr}\left(g^{k} \mid L_{(\beta, b)}\right) E^{k(\beta, b)}\right) \\
\times & \prod_{(\beta, b) \in \Gamma \times \mathcal{A}_{1}} \exp \left(-\sum_{k=1}^{\infty} \frac{1}{k} \operatorname{tr}\left(g^{2 k} \mid L_{(\beta, b)}\right) E^{2 k(\beta, b)}\right) \\
= & \prod_{(\alpha, a) \in \Gamma \times \mathcal{A}} \exp \left(-\sum_{k=1}^{\infty} \frac{1}{k} \psi(a) \operatorname{tr}\left(g^{k} \mid L_{(\alpha, a)}\right) E^{k(\alpha, a)}\right) \\
\times & \prod_{(\alpha, a)=2(\beta, b)} \exp \left(-\sum_{k=1}^{\infty} \frac{1}{k} \operatorname{tr}\left(g^{2 k} \mid L_{(\beta, b)}\right) E^{k(\alpha, a)}\right) .
\end{aligned}
$$

Hence, for any $g \in G$, the generalized denominator identity for the free Lie super- 
algebra $\mathfrak{L}$ is the same as

$$
\begin{aligned}
\prod_{(\alpha, a) \in \Gamma \times \mathcal{A}} & \exp \left(-\sum_{k=1}^{\infty} \frac{1}{k} \operatorname{str}\left(g^{k} \mid \mathfrak{L}_{(\alpha, a)}\right) E^{k(\alpha, a)}\right) \\
= & 1-\sum_{(\alpha, a) \in \Gamma \times \mathcal{A}} \operatorname{str}\left(g \mid V_{(\alpha, a)}\right) E^{(\alpha, a)} \\
= & \prod_{(\alpha, a) \in \Gamma \times \mathcal{A}} \exp \left(-\sum_{k=1}^{\infty} \frac{1}{k} \psi(a) \operatorname{tr}\left(g^{k} \mid L_{(\alpha, a)}\right) E^{k(\alpha, a)}\right) \\
& \times \prod_{\substack{(\alpha, a)=2(\beta, b) \\
(\beta, b) \in \Gamma \times \mathcal{A}_{1}}} \exp \left(-\sum_{k=1}^{\infty} \frac{1}{k} \operatorname{tr}\left(g^{2 k} \mid L_{(\beta, b)}\right) E^{k(\alpha, a)}\right),
\end{aligned}
$$

which yields:

Proposition 4.2 Let $\mathcal{A}$ be a countable abelian group with a coloring map $\theta$ and $\Gamma$ be a countable abelian semigroup satisfying the finiteness condition given in Section 2. For a $(\Gamma \times \mathcal{A})$-graded superspace $V=\bigoplus_{(\alpha, a) \in \Gamma \times \mathcal{A}} V_{(\alpha, a)}$ with finite dimensional homogeneous subspaces, let $L=\bigoplus_{(\alpha, a) \in \Gamma \times \mathcal{A}} L_{(\alpha, a)}$ be the free Lie algebra generated by $V$, and let $\mathfrak{L}=\bigoplus_{(\alpha, a) \in \Gamma \times \mathcal{A}} \mathfrak{L}_{(\alpha, a)}$ be the free Lie superalgebra generated by $V$. Suppose that a group $G$ acts on $V$ preserving the $(\Gamma \times \mathcal{A})$-gradation. Then, for any $g \in G$, we have

$$
\operatorname{str}\left(g \mid \mathfrak{L}_{(\alpha, a)}\right)=\psi(a) \operatorname{tr}\left(g \mid L_{(\alpha, a)}\right)+\sum_{\substack{(\alpha, a)=2(\beta, b) \\(\beta, b) \in \Gamma \times \mathcal{A}_{1}}} \operatorname{tr}\left(g^{2} \mid L_{(\beta, b)}\right) .
$$

Remark. When $g=1$, we recover the superdimension formula

$$
\operatorname{sdim} \mathfrak{L}_{(\alpha, a)}=\psi(a) \operatorname{dim} L_{(\alpha, a)}+\sum_{\substack{(\alpha, a)=2(\beta, b) \\(\beta, b) \in \Gamma \times \mathcal{A}_{1}}} \operatorname{dim} L_{(\beta, b)},
$$

which was obtained in 40.

\section{Decomposition of Free Lie Superalgebras}

In this section, we investigate the structure of the free Lie superalgebra generated by the natural representation of the general linear Lie superalgebra $g l(k, l)$. For $k, l \in \mathbb{Z}_{\geq 0}$, let $\mathfrak{L}=g l(k, l)$ be the space of all $(k+l) \times(k+l)$ matrices, and set

$$
\mathfrak{L}_{0}=\left\{\left(\begin{array}{cc}
A & 0 \\
0 & D
\end{array}\right) \mid A \text { is a } k \times k \text { matrix and } D \text { is an } l \times l \text { matrix }\right\}
$$




$$
\mathfrak{L}_{1}=\left\{\left(\begin{array}{cc}
0 & B \\
C & 0
\end{array}\right) \mid B \text { is a } k \times l \text { matrix and } C \text { is an } l \times k \text { matrix }\right\} .
$$

Then $\mathfrak{L}=\mathfrak{L}_{0} \oplus \mathfrak{L}_{1}$ and $\mathfrak{L}$ becomes a $\mathbb{Z}_{2}$-graded Lie superalgebra called the general linear Lie superalgebra with the superbracket defined by

$$
[X, Y]=X Y-(-1)^{\alpha \beta} Y X \quad \text { for } X \in \mathfrak{L}_{\alpha}, Y \in \mathfrak{L}_{\beta}, \alpha, \beta \in \mathbb{Z}_{2} .
$$

A $\mathbb{Z}_{2}$-graded superspace $V=V_{0} \oplus V_{1}$ is an $\mathfrak{L}$-module if there is a $\mathbb{Z}_{2}$-graded Lie superalgebra homomorphism

$$
\phi: \mathfrak{L} \longrightarrow g l(V) \cong g l\left(k^{\prime}, l^{\prime}\right), \text { where } k^{\prime}=\operatorname{dim} V_{0}, l^{\prime}=\operatorname{dim} V_{1} .
$$

For $\mathfrak{L}$-modules $V^{1}, \cdots, V^{n}$, the tensor product $V^{1} \otimes \cdots \otimes V^{n}$ becomes an $\mathfrak{L}$-module with the action of $\mathfrak{L}$ defined by

$$
x \cdot\left(v_{1} \otimes \cdots \otimes v_{n}\right)=\sum_{i=1}^{n}(-1)^{\operatorname{deg} x\left(\sum_{j<i} \operatorname{deg} v_{j}\right)} v_{1} \otimes \cdots \otimes x \cdot v_{i} \otimes \cdots \otimes v_{n}
$$

for $x \in \mathfrak{L}, v_{i} \in V^{i}$.

We recall some of the basic theory of symmetric functions. A partition is any (finite or infinite) sequence $\lambda=\left(\lambda_{1}, \lambda_{2}, \cdots\right)$ of non-negative integers in decreasing orders $\lambda_{1} \geq \lambda_{2} \geq \cdots$ and containing only finitely many non-zero terms. The non-zero $\lambda_{i}$ 's are called the parts of $\lambda$. The number of parts is the length of $\lambda$, denoted by $l(\lambda)$, and the sum of parts is the weight of $\lambda$, denoted by $|\lambda|$. If $|\lambda|=n$, then we say that $\lambda$ is a partition of $n$, denote by $\lambda \vdash n$. We also use the notation which indicates the number of times each integer occurs as a part. That is, $\lambda=\left(1^{m_{1}}, 2^{m_{2}}, \cdots\right)$ means that $i$ appears as a part of $\lambda$ exactly $m_{i}$ times. Each partition $\lambda$ uniquely determines a Young diagram. The conjugate of a partition $\lambda$ is the partition $\lambda^{\prime}$ whose diagram is the transpose of that of $\lambda$ (i.e., the diagram obtained by reflection in the main diagonal). There is a partial ordering on the set of parititions of $n$, called the natural (partial) ordering or the dominance relation, which is defined as follows:

$$
\lambda \geq \mu \text { if and only if } \sum_{i=1}^{k} \lambda_{i} \geq \sum_{i=1}^{k} \mu_{i} \text { for all } k \geq 1 .
$$

Let $x_{1}, \cdots, x_{k}$ be the indeterminates and $\lambda$ a partition of $n$ with $l(\lambda) \leq k$. A Schur function corresponding to $\lambda$ is a symmetric function in $x_{1}, \cdots, x_{k}$ defined by

$$
S_{\lambda}(x)=S_{\lambda}\left(x_{1} \cdots, x_{k}\right)=\frac{\left|x_{i}^{\lambda_{j}+n-j}\right|}{\left|x_{i}^{n-j}\right|} .
$$

For a partition $\mu=\left(\mu_{1}, \mu_{2}, \cdots\right)$, we define

$$
p_{\mu}(x)=p_{\mu}\left(x_{1}, \cdots, x_{k}\right)=\prod p_{\mu_{i}}\left(x_{1}, \cdots, x_{k}\right),
$$


where $p_{\mu_{i}}\left(x_{1}, \cdots, x_{k}\right)=\sum x_{j}^{\mu_{i}}$ is the $\mu_{i}$-th power sum. It is known that the set of Schur functions $\left\{S_{\lambda}(x) \mid l(\lambda) \leq k\right\}$ (resp. $\left.\left\{p_{\lambda}(x) \mid l(\lambda) \leq k\right\}\right)$ forms a $\mathbb{Z}$-basis (resp. $\mathbb{Q}$-basis) of the ring of symmetric functions in $x_{1}, \cdots, x_{k}$, and $\left\{S_{\lambda}(x) \mid l(\lambda) \leq k, \lambda \vdash n\right\}$ (resp. $\left\{p_{\lambda}(x) \mid l(\lambda) \leq k, \lambda \vdash n\right\}$ ) forms a $\mathbb{Z}$-basis (resp. $\mathbb{Q}$-basis) of the subspace of symmetric functions in $x_{1}, \cdots, x_{k}$ with homogeneous degree $n$. The functions $S_{\lambda}(x)$ and $p_{\lambda}(x)$ are related as follows:

Lemma 5.1 (46]) For each partition $\rho$ of $n$, we have

$$
p_{\rho}(x)=\sum_{\lambda \vdash n} \chi_{\lambda}^{\rho} S_{\lambda}(x)
$$

where $\chi_{\lambda}^{\rho}$ is the character value of the irreducible representation of $S_{n}$ corresponding to $\lambda$ at the conjugacy class of type $\rho$.

A partition $\lambda$ is called a $(k, l)$-hook partition if $\lambda_{k+1} \leq l$. Set $H(k, l ; n)=\{\lambda \vdash$ $\left.n \mid \lambda_{k+1} \leq l\right\}$. For convenience, we will often write $H$ for $H(k, l ; n)$. For $\lambda \in H(k, l ; n)$, a $(k, l)$-hook Schur function in $x_{1}, \cdots, x_{k}, y_{1}, \cdots, y_{l}$ corresponding to $\lambda$ is a function defined by

$$
H S_{\lambda}(x, y)=H S_{\lambda}\left(x_{1}, \cdots, x_{k}, y_{1}, \cdots, y_{l}\right)=\sum_{\mu<\lambda} S_{\mu}(x) S_{\lambda^{\prime} / \mu^{\prime}}(y)
$$

where $S_{\lambda^{\prime} / \mu^{\prime}}(y)$ is the skew Schur function corresponding to $\lambda^{\prime}, \mu^{\prime}$ (cf. [8], [46], [52]). Equivalently,

$$
H S_{\lambda}(x, y)=\sum_{\mu<\lambda} S_{\mu}(x)\left(\sum_{\nu} N_{\mu^{\prime} \nu}^{\lambda^{\prime}} S_{\nu}(y)\right)
$$

where $N_{\mu^{\prime} \nu}^{\lambda^{\prime}}$ is the Littlewood-Richardson coefficient corresponding to $\lambda^{\prime}, \mu^{\prime}, \nu$. The combinatorial interpretation of the Littlewood-Richardson coefficients is given as follows: for given partitions $\lambda, \mu, \nu$ satisfying $|\lambda|=|\mu|+|\nu|$ and $\lambda_{i} \geq \mu_{i}$ for all $i, N_{\mu \nu}^{\lambda}$ is the number of ways the Young diagram for $\mu$ can be expanded to the Young diagram for $\lambda$ by a strict $\nu$-expansion (if the above conditions do not hold, $N_{\mu \nu}^{\lambda}=0$ ). For a partition $\nu=\left(\nu_{1}, \cdots, \nu_{k}\right)$, a $\nu$-expansion of a Young diagram for $\mu$ is obtained by first adding $\nu_{1}$ boxes to the Young diagram for $\mu$ with no two boxes in the same column and putting the integer 1 in each of these $\nu_{1}$ boxes; then adding similarly $\nu_{2}$ boxes with a 2 , continuing until finally $\nu_{k}$ boxes are added with the integer $k$. The expansion is called strict if, when the integers in the boxes are listed from right to left, starting with the top row and working down, and one looks at the first $t$ entries in this list (for any $t$ between 1 and $|\nu|$ ), each integer $p$ between 1 and $k-1$ occurs at least as many times as the next integer $p+1$.

In [8], Berele and Regev showed that, for a $\mathbb{Z}_{2}$-graded superspace $V=V_{0} \oplus V_{1}$ viewed as a $g l(k, l)$-module $\left(k=\operatorname{dim} V_{0}, l=\operatorname{dim} V_{1}\right), V^{\otimes n}$ is completely reducible 
as a $g l(k, l)$-module and its irreducible components are parametrized by $(k, l)$-hook partitions $\lambda$, denoted by $V_{\lambda}$.

Let $\mathfrak{L}(V)$ be the free Lie superalgebra generated by $V$. Set $\mathfrak{L}(V)_{(n, 0)}=\mathfrak{L}(V) \cap$ $\left(V^{\otimes n}\right)_{0}, \mathfrak{L}(V)_{(n, 1)}=\mathfrak{L}(V) \cap\left(V^{\otimes n}\right)_{1}$ and $\mathfrak{L}_{n}(V)=\mathfrak{L}(V)_{(n, 0)} \oplus \mathfrak{L}(V)_{(n, 1)}$. Thus $\mathfrak{L}(V)$ has an $\left(\mathbb{N} \times \mathbb{Z}_{2}\right)$-gradation: $\mathfrak{L}(V)=\bigoplus_{\mathbb{N} \times \mathbb{Z}_{2}} \mathfrak{L}(V)_{(n, \alpha)}$. The homogeneous subspace $\mathfrak{L}_{n}(V)$ is a $g l(k, l)$-submodule of $V^{\otimes n}$, where the action of $x \in g l(k, l)$ induced from $V^{\otimes n}$ is given by

$$
\begin{aligned}
& x \cdot\left[v_{1},\left[v_{2},\left[\cdots\left[v_{n-1}, v_{n}\right] \cdots\right]\right]\right] \\
& =\sum_{i=1}^{n}(-1)^{\operatorname{deg} x\left(\sum_{j<i} \operatorname{deg} v_{j}\right)}\left[v_{1},\left[\cdots\left[x v_{i}\left[\cdots\left[v_{n-1}, v_{n}\right] \cdots\right]\right]\right]\right]
\end{aligned}
$$

and it is completely reducible with its irreducible components parametrized by $\lambda^{\prime} s \in H$.

¿From now on, we simply write $\mathfrak{L}_{n}, \mathfrak{L}_{(n, 0)}$ and $\mathfrak{L}_{(n, 1)}$ for $\mathfrak{L}_{n}(V), \mathfrak{L}(V)_{(n, 0)}$ and $\mathfrak{L}(V)_{(n, 1)}$, respectively. For each $\lambda \in H(k, l ; n)$, let $c_{\lambda}$ be the multiplicity of $V_{\lambda}$ in $\mathfrak{L}_{n}$. To compute $c_{\lambda}$, we would like to apply our supertrace formula. By exponentiating the action of the Lie algebra $g l(k, l)_{0}=g l(k) \times g l(l)$ on $V$, the group $G L(k) \times G L(l)$ acts on $V$, and hence $V^{\otimes n}$ and $\mathfrak{L}_{n}(V)$ become $G L(k) \times G L(l)$-modules. We first recall:

Proposition $5.2([8])$ Let $\lambda \in H(k, l ; n)$ and $V_{\lambda}$ be the corresponding irreducible representation of $g l(k, l)$. Suppose that $g=\left(g_{0}, g_{1}\right) \in G L(k) \times G L(l)$ has eigenvalues $x_{1}, \cdots, x_{k}$ for $g_{0}$ and $y_{1}, \cdots, y_{l}$ for $g_{1}$. Then we have

$$
\operatorname{tr}\left(g \mid V_{\lambda}\right)=H S_{\lambda}(x, y)
$$

Our supertrace formula (4.2) for free Lie superalgebras yields

$$
\operatorname{str}\left(g \mid \mathfrak{L}_{(n, \alpha)}\right)=\sum_{d \mid(n, \alpha)} \frac{1}{d} \mu(d) \sum_{s \in T((n, \alpha) / d)} \frac{(|s|-1) !}{s !} \operatorname{str}\left(g_{0}^{d} \mid V_{0}\right)^{s_{0}} \operatorname{str}\left(g_{1}^{d} \mid V_{1}\right)^{s_{1}}
$$

where

$$
\begin{aligned}
T(m, 0) & =\left\{\left(s_{0}, s_{1}\right) \mid s_{0}+s_{1}=m, s_{1} \equiv 0(\bmod 2)\right\} \\
T(m, 1) & =\left\{\left(s_{0}, s_{1}\right) \mid s_{0}+s_{1}=m, s_{1} \equiv 1(\bmod 2)\right\}
\end{aligned}
$$


Hence, for $g=\left(g_{0}, g_{1}\right) \in G L(k) \times G L(l)$, we have

$$
\begin{aligned}
& \operatorname{tr}\left(g \mid \mathfrak{L}_{(n, 0)}\right)=\operatorname{str}\left(g \mid \mathfrak{L}_{(n, 0)}\right) \\
& =\sum_{d \mid(n, 0)} \frac{1}{d} \mu(d) \sum_{s \in T((n, 0) / d)} \frac{(|s|-1) !}{s !} \operatorname{str}\left(g_{0}^{d} \mid V_{0}\right)^{s_{0}} \operatorname{str}\left(g_{1}^{d} \mid V_{1}\right)^{s_{1}} \\
& =\sum_{d \mid(n, 0)} \frac{1}{d} \mu(d) \sum_{s \in T((n, 0) / d)} \frac{(|s|-1) !}{s !}(-1)^{s_{1}} p_{d}(x)^{s_{0}} p_{d}(y)^{s_{1}} \\
& =\sum_{d \mid(n, 0)} \frac{1}{d} \mu(d) \sum_{s \in T((n, 0) / d)} \frac{(|s|-1) !}{s !}(-1)^{s_{1}}\left(\sum_{\lambda \vdash d s_{0}} \chi_{\lambda}^{\left(d^{s_{0}}\right)} S_{\lambda}(x)\right)\left(\sum_{\mu \vdash d s_{1}} \chi_{\mu}^{\left(d^{s_{1}}\right)} S_{\mu}(y)\right) \\
& =\sum_{d \mid(n, 0)} \frac{1}{d} \mu(d) \sum_{s \in T((n, 0) / d)} \frac{(|s|-1) !}{s !}(-1)^{s_{1}}\left(\sum_{\substack{\lambda \vdash d s_{0} \\
\mu \vdash d s_{1}}} \chi_{\lambda}^{\left(d^{s_{0}}\right)} \chi_{\mu}^{\left(d^{s_{1}}\right)} S_{\lambda}(x) S_{\mu}(y)\right),
\end{aligned}
$$

and

$$
\begin{aligned}
\operatorname{tr} & \left(g \mid \mathfrak{L}_{(n, 1)}\right)=-\operatorname{str}\left(g \mid \mathfrak{L}_{(n, 1)}\right) \\
= & -\sum_{d \mid(n, 1)} \frac{1}{d} \mu(d) \sum_{s \in T((n, 1) / d)} \frac{(|s|-1) !}{s !} \operatorname{str}\left(g_{0}^{d} \mid V_{0}\right)^{s_{0}} \operatorname{str}\left(g_{1}^{d} \mid V_{1}\right)^{s_{1}} \\
= & -\sum_{\substack{d \mid n \\
d: \text { odd }}} \frac{1}{d} \mu(d) \sum_{s \in T((n, 1) / d)} \frac{(|s|-1) !}{s !}(-1)^{s_{1}} \operatorname{tr}\left(g_{0}^{d} \mid V_{0}\right)^{s_{0}} \operatorname{tr}\left(g_{1}^{d} \mid V_{1}\right)^{s_{1}} \\
= & \sum_{\substack{d \mid n \\
d: \text { odd }}} \frac{1}{d} \mu(d) \sum_{s \in T((n, 1) / d)} \frac{(|s|-1) !}{s !} p_{d}(x)^{s_{0}} p_{d}(y)^{s_{1}} \\
= & \sum_{\substack{d \mid n \\
d: \text { odd }}} \frac{1}{d} \mu(d) \sum_{s \in T((n, 1) / d)} \frac{(|s|-1) !}{s !}\left(\sum_{\lambda \vdash d s_{0}} \chi_{\lambda}^{\left(d^{s_{0}}\right)} S_{\lambda}(x)\right)\left(\sum_{\mu \vdash d s_{1}} \chi_{\mu}^{\left(d^{s_{1}}\right)} S_{\mu}(y)\right) \\
= & \sum_{\substack{d \mid n \\
d: \text { odd }}} \frac{1}{d} \mu(d) \sum_{s \in T((n, 1) / d)} \frac{(|s|-1) !}{s !}\left(\sum_{\substack{\lambda \vdash d s_{0} \\
\mu \vdash d s_{1}}} \chi_{\lambda}^{\left(d^{s_{0}}\right)} \chi_{\mu}^{\left(d^{s_{1}}\right)} S_{\lambda}(x) S_{\mu}(y)\right)
\end{aligned}
$$


It follows that

$$
\begin{aligned}
& \operatorname{tr}\left(g \mid \mathfrak{L}_{n}\right)=\operatorname{tr}\left(g \mid \mathfrak{L}_{(n, 0)}\right)+\operatorname{tr}\left(g \mid \mathfrak{L}_{(n, 1)}\right) \\
& =\sum_{\substack{d \mid n \\
d: \text { odd }}} \frac{1}{d} \mu(d) \sum_{s_{0}+s_{1}=n / d} \frac{(|s|-1) !}{s !}\left(\sum_{\substack{\lambda \vdash d s_{0} \\
\mu \vdash d s_{1}}} \chi_{\lambda}^{\left(d^{\left.s_{0}\right)}\right)} \chi_{\mu}^{\left(d^{s_{1}}\right)} S_{\lambda}(x) S_{\mu}(y)\right) \\
& +\sum_{\substack{d \mid n \\
d: \operatorname{even}}} \frac{1}{d} \mu(d) \sum_{s_{0}+s_{1}=n / d}(-1)^{s_{1}} \frac{(|s|-1) !}{s !}\left(\sum_{\substack{\lambda \vdash d s_{0} \\
\mu \vdash d s_{1}}} \chi_{\lambda}^{\left(d^{s_{0}}\right)} \chi_{\mu}^{\left(d^{s_{1}}\right)} S_{\lambda}(x) S_{\mu}(y)\right) \\
& =\sum_{\substack{l(\lambda) \leq k \\
l(\mu) \leq l \\
|\lambda|+|\mu|=n}} a_{\lambda \mu} S_{\lambda}(x) S_{\mu}(y),
\end{aligned}
$$

where

$$
\begin{aligned}
a_{\lambda \mu}= & \sum_{\begin{array}{c}
d \mid n \\
d: \text { odd }
\end{array}} \frac{\mu(d)}{d} \sum_{\substack{s_{0}+s_{1}=n / d \\
d s_{0}=|\lambda|}} \frac{(|s|-1) !}{s !} \chi_{\lambda}^{\left(d^{s_{0}}\right)} \chi_{\mu}^{\left(d^{s_{1}}\right)} \\
& +\sum_{\substack{d \mid n \\
d: \text { :even }}} \frac{\mu(d)}{d} \sum_{\substack{s_{0}+s_{1}=n / d \\
d s_{0}=|\lambda|}}(-1)^{s_{1}} \frac{(|s|-1) !}{s !} \chi_{\lambda}^{\left(d^{s_{0}}\right)} \chi_{\mu}^{\left(d^{s_{1}}\right)} \\
= & \frac{1}{n} \sum_{d|| \lambda|,| \mu \mid} \mu(d) \frac{(n / d) !}{(|\lambda| / d) !(|\mu| / d) !}(-1)^{(d-1) \frac{|\mu|}{d}} \chi_{\lambda}^{\left(d^{|\lambda| / d}\right)} \chi_{\mu}^{\left(d^{|\mu| / d}\right)} .
\end{aligned}
$$

Note that $a_{\lambda \mu}$ is the multiplicity of $W_{\lambda} \otimes W_{\mu}$ in $\mathfrak{L}_{n}$, where $W_{\lambda}$ (resp. $W_{\mu}$ ) is the irreducible $G L(k)$-module (resp. $G L(l)$-module) corresponding to $\lambda$ (resp. $\mu$ ). Recall that $c_{\lambda}$ is the multiplicity of $V_{\lambda}$ in $\mathfrak{L}_{n}$. By Proposition 5.2, we have

$$
\operatorname{tr}\left(g \mid \mathfrak{L}_{n}\right)=\sum_{\lambda \in H} c_{\lambda} H S_{\lambda}(x, y) .
$$

Therefore, we obtain:

Proposition 5.3 If $\lambda=\left(\tau_{1}, \tau_{2}, \cdots, \tau_{k}, \tau_{k+1}, \cdots\right)$, then we have

$$
c_{\lambda}=a_{\lambda_{0} \lambda_{1}}-\sum_{\substack{\mu \in H-\{\lambda\} \\ \mu_{0}>\lambda_{0}}} c_{\mu} N_{\lambda_{0}^{\prime} \lambda_{1}}^{\mu^{\prime}},
$$

where $\lambda_{0}=\left(\tau_{1}, \cdots, \tau_{k}\right)$ and $\lambda_{1}=\left(\tau_{k+1}, \cdots\right)^{\prime}$. 
Proof. Comparing the equations (5.13) and (5.14), we have

$$
\begin{aligned}
& \sum_{\mu \in H} c_{\mu} H S_{\mu}(x, y)=c_{\lambda} H S_{\lambda}(x, y)+\sum_{\mu \neq \lambda} c_{\mu} H S_{\mu}(x, y) \\
& =c_{\lambda}\left(\sum_{\nu<\lambda} S_{\nu}(x) S_{\lambda^{\prime} / \nu^{\prime}}(y)\right)+\sum_{\mu \neq \lambda} c_{\mu} H S_{\mu}(x, y) \\
& =c_{\lambda}\left(S_{\lambda_{0}}(x) S_{\lambda_{1}}(y)+(*)\right)+\sum_{\mu \neq \lambda} c_{\mu}\left(\sum_{\tau<\mu} S_{\tau}(x) \sum_{\sigma} N_{\tau^{\prime} \sigma}^{\mu^{\prime}} S_{\sigma}(y)\right) \\
& =\left(c_{\lambda}+\sum_{\substack{\mu \in H \\
\mu_{0}>\lambda_{0}}} c_{\mu} N_{\lambda_{0}^{\prime} \lambda_{1}}^{\mu^{\prime}}\right) S_{\lambda_{0}}(x) S_{\lambda_{1}}(y)+(*)^{\prime} \\
& =\sum_{\substack{l(\lambda) \leq k \\
l(\mu) \leq l \\
|\lambda|+|\mu|=n}} a_{\lambda \mu} S_{\lambda}(x) S_{\mu}(y) \text {. }
\end{aligned}
$$

Since $\left\{S_{\lambda}(x) S_{\mu}(y) \mid l(\lambda) \leq k, l(\mu) \leq l\right\}$ is linearly independent ([ø]), we obtain

$$
a_{\lambda_{0} \lambda_{1}}=c_{\lambda}+\sum_{\substack{\mu \in H-\{\lambda\} \\ \mu_{0}>\lambda_{0}}} c_{\mu} N_{\lambda_{0}^{\prime} \lambda_{1}}^{\mu^{\prime}},
$$

which completes the proof.

If $l(\lambda) \leq k\left(\right.$ or $\left.\left|\lambda_{0}\right|=n\right)$ for $\lambda \in H(k, l ; n)$, then (5.14) yields

$$
c_{\lambda}=a_{\lambda_{0} \lambda_{1}}=\frac{1}{n} \sum_{d \mid n} \mu(d) \chi_{\lambda}^{\left(d^{n / d}\right)}
$$

If all $c_{\lambda}$ 's are known for $m \leq\left|\lambda_{0}\right| \leq n$, then for any $\lambda \in H(k, l ; n)$ with $\left|\lambda_{0}\right|=m-1$, we have

$$
\begin{array}{r}
c_{\lambda}=a_{\lambda_{0} \lambda_{1}}-\sum_{\substack{\mu \in H-\{\lambda\} \\
\mu_{0}>\lambda_{0}}} c_{\mu} N_{\lambda_{0}^{\prime} \lambda_{1}}^{\mu^{\prime}} \\
=a_{\lambda_{0} \lambda_{1}}-\sum_{\substack{\mu \in H \\
\mu_{0}>\lambda_{0} \\
\left|\mu_{0}\right| \geq m}} c_{\mu} N_{\lambda_{0}^{\prime} \lambda_{1}}^{\mu^{\prime}},
\end{array}
$$

since $\mu_{0}>\lambda_{0}$ and $\left|\mu_{0}\right|=m-1$ imply $\mu_{0}=\lambda_{0}$, and in this case, $N_{\lambda_{0}^{\prime} \lambda_{1}}^{\mu^{\prime}}=0$ unless $\lambda=\mu$ (cf. [46]). Following the above inductive step, we can completely determine the value of all $c_{\lambda}$ for $\lambda \in H(k, l ; n)$ :

Proposition 5.4 Under the above hypothesis, we have

$$
\mathfrak{L}_{n}(V)=\bigoplus_{\lambda \in H(k, l ; n)} V_{\lambda}^{\oplus c_{\lambda}}
$$


where $V_{\lambda}$ is the irreducible $g l(k, l)$-module corresponding to $\lambda$ and $c_{\lambda}$ is determined by (5.14).

Remark. Note that the multiplicity $c_{\lambda}$ is given explicitly in closed form when $l(\lambda) \leq k$ and is given by a recursive formula otherwise.

Corollary 5.5 (cf. [3], 23]) Under the above hypothesis, if $V_{1}=0$, then we have

$$
\begin{aligned}
\mathfrak{L}_{n}(V) & =\bigoplus_{\substack{\lambda \vdash n \\
l(\lambda) \leq k}} V_{\lambda}^{\oplus c_{\lambda}}, \\
c_{\lambda} & =\frac{1}{n} \sum_{d \mid n} \mu(d) \chi_{\lambda}^{\left(d^{n / d}\right)},
\end{aligned}
$$

where $V_{\lambda}$ is the irreducible $g l(k)$-module corresponding to $\lambda$.

\section{Generalized Kac-Moody Superalgebras}

The generalized Kac-Moody superalgebras arise naturally in the context of Monstrous Moonshine ([5], [6]), automorphic forms with infinite product expansions ([7], [18]20]), and the string theory (22]). In this section, we give a conjectural formula for the homology modules over (the negative part of) generalized Kac-Moody superalgebras, and derive a closed form supertrace formula for generalized Kac-Moody superalgebras with group actions.

Let $I$ be a countable (possibly infinite) index set. A real square matrix $A=\left(a_{i j}\right)_{i, j \in I}$ is called a Borcherds-Cartan matrix if it satisfies: (i) $a_{i i}=2$ or $a_{i i} \leq 0$ for all $i \in I$, (ii) $a_{i j} \leq 0$ if $i \neq j$, and $a_{i j} \in \mathbb{Z}$ if $a_{i i}=2$, (iii) $a_{i j}=0$ implies $a_{j i}=0$. We say that an index $i$ is real if $a_{i i}=2$ and imaginary if $a_{i i} \leq 0$. We denote by $I^{r e}=\left\{i \in I \mid a_{i i}=2\right\}$, $I^{i m}=\left\{i \in I \mid a_{i i} \leq 0\right\}$. Let $\underline{m}=\left(m_{i} \in \mathbb{Z}_{>0} \mid i \in I\right)$ be a sequence of positive integers such that $m_{i}=1$ for all $i \in I^{r e}$. We call $\underline{m}$ the charge of $A$. In this paper, we assume that the Borcherds-Cartan matrix $A$ is symmetrizable, i.e., there is a diagonal matrix $D=\operatorname{diag}\left(s_{i} \mid i \in I\right)$ with $s_{i}>0(i \in I)$ such that $D A$ is symmetric.

Let $C=\left(\theta_{i j}\right)_{i, j \in I}$ be a complex matrix satisfying $\theta_{i j} \theta_{j i}=1$ for all $i, j \in I$. Thus we have $\theta_{i i}= \pm 1$ for all $i \in I$. We call $i \in I$ an even index if $\theta_{i i}=1$ and an odd index if $\theta_{i i}=-1$. We denote by $I^{\text {even }}$ (resp. $I^{\text {odd }}$ ) the set of all even (resp. odd) indices. We say that a Borcherds-Cartan matrix $A=\left(a_{i j}\right)_{i, j \in I}$ is colored by $C$ if $a_{i i}=2$ and $\theta_{i i}=-1$ imply $a_{i j}$ are even integers for all $j \in I$. In this case, the matrix $C$ is called a coloring matrix of $A$. 
Let $\mathfrak{h}=\left(\bigoplus_{i \in I} \mathbb{C} h_{i}\right) \oplus\left(\bigoplus_{i \in I} \mathbb{C} d_{i}\right)$ be a complex vector space with a basis $\left\{h_{i}, d_{i} \mid i \in\right.$ $I\}$, and for each $i \in I$ define a linear functional $\alpha_{i} \in \mathfrak{h}^{*}$ by

$$
\alpha_{i}\left(h_{j}\right)=a_{j i}, \quad \alpha_{i}\left(d_{j}\right)=\delta_{i j} \text { for all } j \in I .
$$

The free abelian group $Q=\bigoplus_{i \in I} \mathbb{Z} \alpha_{i}$ generated by $\alpha_{i}$ 's $(i \in I)$ is called the root lattice associated with $A$. Let $\Pi=\left\{\alpha_{i} \mid i \in I\right\}$ and $B$ be a basis of $\mathfrak{h}^{*}$ extending $\Pi$. Set $B^{\prime}=B \backslash \Pi$. Since $A$ is assumed to be symmetrizable, there is a symmetric bilinear form $(\mid)$ on $\mathfrak{h}^{*}$ defined by

$$
\begin{aligned}
& \left(\alpha_{i} \mid \alpha_{j}\right)=s_{i} a_{i j} \text { for } i, j \in I, \\
& \left(\lambda \mid \alpha_{i}\right)=\lambda\left(s_{i} h_{i}\right) \text { for } \lambda \in B^{\prime}, \\
& (\lambda \mid \mu)=0 \text { for } \lambda, \mu \in B^{\prime}
\end{aligned}
$$

(cf. see, for example, 24]). Let $Q^{+}=\sum_{i \in I} \mathbb{Z}_{\geq 0} \alpha_{i}$ and $Q^{-}=-Q^{+}$. There is a partial ordering $\geq$ on $Q$ given by $\lambda \geq \mu$ if and only if $\lambda-\mu \in Q^{+}$. The coloring matrix $C=\left(\theta_{i j}\right)_{i, j \in I}$ defines a bimultiplicative map $\theta: Q \times Q \rightarrow \mathbb{C}^{\times}$on $Q$ by

$$
\begin{aligned}
& \theta\left(\alpha_{i}, \alpha_{j}\right)=\theta_{i j} \text { for all } i, j \in I, \\
& \theta(\alpha+\beta, \gamma)=\theta(\alpha, \gamma) \theta(\beta, \gamma), \\
& \theta(\alpha, \beta+\gamma)=\theta(\alpha, \beta) \theta(\alpha, \gamma)
\end{aligned}
$$

for all $\alpha, \beta, \gamma \in Q$. Note that, since $\theta_{i j} \theta_{j i}=1$ for all $i, j \in I, \theta$ satisfies

$$
\theta(\alpha, \beta) \theta(\beta, \alpha)=1 \text { for all } \alpha, \beta \in Q \text {. }
$$

That is, $\theta$ is a coloring map on $Q$. In particular, $\theta(\alpha, \alpha)= \pm 1$ for all $\alpha \in Q$. We say $\alpha \in Q$ is even if $\psi(\alpha)=\theta(\alpha, \alpha)=1$ and odd if $\psi(\alpha)=\theta(\alpha, \alpha)=-1$.

The generalized Kac-Moody superalgebra $\mathfrak{g}=\mathfrak{g}(A, \underline{m}, C)$ associated with a symmetrizable Borcherds-Cartan matrix $A=\left(a_{i j}\right)_{i, j \in I}$ of charge $\underline{m}=\left(m_{i} \mid i \in I\right)$ with a coloring matrix $C=\left(\theta_{i j}\right)_{i, j \in I}$ is the $\theta$-colored Lie superalgebra generated by the elements $h_{i}, d_{i}(i \in I), e_{i k}, f_{i k}\left(i \in I, k=1,2, \cdots, m_{i}\right)$ with the defining relations:

$$
\begin{aligned}
& {\left[h_{i}, h_{j}\right]=\left[h_{i}, d_{j}\right]=\left[d_{i}, d_{j}\right]=0,} \\
& {\left[h_{i}, e_{j l}\right]=a_{i j} e_{j l}, \quad\left[h_{i}, f_{j l}\right]=-a_{i j} f_{j l},} \\
& {\left[d_{i}, e_{j l}\right]=\delta_{i j} e_{j l}, \quad\left[d_{i}, f_{j l}\right]=-\delta_{i j} f_{j l},} \\
& {\left[e_{i k}, f_{j l}\right]=\delta_{i j} \delta_{k l} h_{i},} \\
& \left(a d e_{i k}\right)^{1-a_{i j}}\left(e_{j l}\right)=\left(a d f_{i k}\right)^{1-a_{i j}}\left(f_{j l}\right)=0 \text { if } a_{i i}=2 \text { and } i \neq j, \\
& {\left[e_{i k}, e_{j l}\right]=\left[f_{i k}, f_{j l}\right]=0 \text { if } a_{i j}=0}
\end{aligned}
$$

for $i, j \in I, k=1, \cdots, m_{i}, l=1, \cdots, m_{j}$.

The abelian subalgebra $\mathfrak{h}=\left(\bigoplus_{i \in I} \mathbb{C} h_{i}\right) \bigoplus\left(\bigoplus_{i \in I} \mathbb{C} d_{i}\right)$ is called the Cartan subalgebra of $\mathfrak{g}$, and the linear functionals $\alpha_{i} \in \mathfrak{h}^{*}(i \in I)$ defined by (6.1) are called the simple roots of $\mathfrak{g}$. For each $i \in I^{r e}$, let $r_{i} \in G L\left(\mathfrak{h}^{*}\right)$ be the simple reflection on $\mathfrak{h}^{*}$ defined by

$$
r_{i}(\lambda)=\lambda-\lambda\left(h_{i}\right) \alpha_{i} \text { for } \lambda \in \mathfrak{h}^{*} .
$$


The subgroup $W$ of $G L\left(\mathfrak{h}^{*}\right)$ generated by the $r_{i}$ 's $\left(i \in I^{r e}\right)$ is called the Weyl group of the generalized Kac-Moody superalgebra $\mathfrak{g}$.

The generalized Kac-Moody superalgebra $\mathfrak{g}=\mathfrak{g}(A, \underline{m}, C)$ has the root space decomposition $\mathfrak{g}=\bigoplus_{\alpha \in Q} \mathfrak{g}_{\alpha}$, where

$$
\mathfrak{g}_{\alpha}=\{x \in \mathfrak{g} \mid[h, x]=\alpha(h) x \text { for all } h \in \mathfrak{h}\} .
$$

Note that $\mathfrak{g}_{\alpha_{i}}=\mathbb{C} e_{i, 1} \oplus \cdots \oplus \mathbb{C} e_{i, m_{i}}$ and $\mathfrak{g}_{-\alpha_{i}}=\mathbb{C} f_{i, 1} \oplus \cdots \oplus \mathbb{C} f_{i, m_{i}}$. We say that $\alpha \in Q^{\times}$ is a root of $\mathfrak{g}$ if $\mathfrak{g}_{\alpha} \neq 0$. The subspace $\mathfrak{g}_{\alpha}$ is called the root space of $\mathfrak{g}$ attached to $\alpha$. A root $\alpha$ is called real if $(\alpha \mid \alpha)>0$ and imaginary if $(\alpha \mid \alpha) \leq 0$. In particular, a simple root $\alpha_{i}$ is real if $a_{i i}=2$ (i.e., $i \in I^{r e}$ ) and imaginary if $a_{i i} \leq 0$ (i.e., $i \in I^{i m}$ ). Note that the imaginary simple roots may have multiplicity $>1$. A root $\alpha>0$ (resp. $\alpha<0$ ) is called positive (resp. negative). One can show that all the roots are either positive or negative. We denote by $\Phi, \Phi^{+}$, and $\Phi^{-}$the set of all roots, positive roots, and negative roots, respectively. We also denote by $\Phi_{0}$ (resp. $\left.\Phi_{1}\right)$ the set of all even (resp. odd) roots of $\mathfrak{g}$. Hence, for example, $\Phi_{0}^{+}$will denote the set of all positive even roots of $\mathfrak{g}$. Define the subalgebras $\mathfrak{g}^{ \pm}=\bigoplus_{\alpha \in \Phi^{ \pm}} \mathfrak{g}_{\alpha}$. Then we have the triangular decomposition:

$$
\mathfrak{g}=\mathfrak{g}^{-} \oplus \mathfrak{h} \oplus \mathfrak{g}^{+} .
$$

We can define a nondegenerate symmetric bilinear form on $\mathfrak{h}$ by

$$
\left(h_{i} \mid h\right)=\frac{1}{s_{i}} \alpha_{i}(h) \text { and }\left(d_{i} \mid d_{j}\right)=0
$$

for all $h \in \mathfrak{h}, i, j \in I$ (cf. [31]). The symmetric bilinear form ( | ) on $\mathfrak{h}$ defined by (6.6) can be extended to a nondegenerate, supersymmetric, and invariant bilinear form on the generalized Kac-Moody superalgebra $\mathfrak{g}$ :

Proposition 6.1 (cf. [24], 30]) Let $\mathfrak{g}=\mathfrak{g}(A, \underline{m}, C)$ be the generalized Kac-Moody superalgebra associated with a Borcherds-Cartan data $(A, \underline{m}, C)$. Then there exists a nondegenerate bilinear form $(\mid)$ on $\mathfrak{g}$ satisfying the following conditions:

(a) The bilinear form $(\mid)$ is supersymmetric, i.e., we have

$$
(x \mid y)=\theta(\beta, \alpha)(y \mid x) \quad \text { for } x \in \mathfrak{g}_{\alpha}, y \in \mathfrak{g}_{\beta} .
$$

(b) The bilinear form $(\mid)$ is invariant, .i.e., we have

$$
([x, y] \mid z)=(x \mid[y, z]) \text { for } x, y, z \in \mathfrak{g} .
$$

(c) The bilinear form $\left.(\mid)\right|_{\mathfrak{h}}$ is given by (6.6).

(d) $\left(\mathfrak{g}_{\alpha} \mid \mathfrak{g}_{\beta}\right)=0$ if $\alpha+\beta \neq 0$.

(e) The root spaces $\mathfrak{g}_{\alpha}$ and $\mathfrak{g}_{-\alpha}$ are nondegenerately paired with respect to ( | ), i.e., the bilinear form $\left.(\mid)\right|_{\mathfrak{g}_{\alpha}+\mathfrak{g}_{-\alpha}}$ is nondegenerate.

(f) If $\phi: Q \rightarrow \mathfrak{h}$ is a linear map satisfying $\phi\left(\alpha_{i}\right)=s_{i} h_{i}$ for all $i \in I$, then we have

$$
[x, y]=\theta(\alpha, \alpha)(x \mid y) \phi(\alpha) \text { for } x \in \mathfrak{g}_{\alpha}, y \in \mathfrak{g}_{-\alpha} .
$$


A $\mathfrak{g}$-module $V$ is called $\mathfrak{h}$-diagonalizable if it admits a weight space decomposition $V=\bigoplus_{\mu \in \mathfrak{h}^{*}} V_{\mu}$, where

$$
V_{\mu}=\{v \in V \mid h \cdot v=\mu(h) v \text { for all } h \in \mathfrak{h}\} .
$$

If $V_{\mu} \neq 0$, then $\mu$ is called a weight of $V$ and $V_{\mu}$ is called the $\mu$-weight space. We denote by $P(V)$ the set of all weights of $V$. When all the weight spaces are finite dimensional, we define the character of $V$ to be

$$
\operatorname{ch} V=\sum_{\mu \in \mathfrak{h}^{*}}\left(\operatorname{dim} V_{\mu}\right) e^{\mu},
$$

where $e^{\mu}$ are the basis elements of the group algebra $\mathbf{C}\left[\mathfrak{h}^{*}\right]$ with the multiplication given by $e^{\mu} e^{\nu}=e^{\mu+\nu}$ for $\mu, \nu \in \mathfrak{h}^{*}$.

We denote by $\mathcal{O}$ the category of $\mathfrak{h}$-diagonalizable $\mathfrak{g}$-modules with finite dimensional weight spaces such that there exist a finite number of linear functionals $\lambda_{1}, \cdots, \lambda_{s}$ satisfying $P(V) \subset \cup_{i=1}^{s} D\left(\lambda_{i}\right)$, where $D(\lambda)=\left\{\mu \in \mathfrak{h}^{*} \mid \mu \leq \lambda\right\}$. The morphisms in $\mathcal{O}$ are the usual $\mathfrak{g}$-module homomorphisms.

The most important example of the $\mathfrak{g}$-modules in category $\mathcal{O}$ may be the class of highest weight modules. An $\mathfrak{h}$-diagonalizable $\mathfrak{g}$-module $V$ is called a highest weight module with highest weight $\lambda \in \mathfrak{h}^{*}$ if there is a nonzero vector $v_{\lambda} \in V$ such that (i) $e_{i k} \cdot v_{\lambda}=0$ for all $i \in I, k=1, \cdots, m_{i}$, (ii) $h \cdot v_{\lambda}=\lambda(h) v_{\lambda}$ for all $h \in \mathfrak{h}$, (iii) $V=U(\mathfrak{g}) \cdot v_{\lambda}$. The vector $v_{\lambda}$ is called a highest weight vector. For a highest weight module $V$ with highest weight $\lambda$, we have (i) $V=U\left(\mathfrak{g}^{-}\right) \cdot v_{\lambda}$, (ii) $V=\bigoplus_{\mu \leq \lambda} V_{\mu}$, $V_{\lambda}=\mathbb{C} v_{\lambda}$, and (iii) $\operatorname{dim} V_{\mu}<\infty$ for all $\mu \leq \lambda$.

Let $\mathfrak{b}^{+}=\mathfrak{h} \oplus \mathfrak{g}^{+}$, and let $\mathbb{C}_{\lambda}$ be the 1-dimensional $\mathfrak{b}^{+}$-module defined by $h \cdot 1=$ $\lambda(h) 1$ for all $h \in \mathfrak{h}$ and $\mathfrak{g}^{+} \cdot 1=0$. The induced module $M(\lambda)=U(\mathfrak{g}) \otimes_{U\left(\mathfrak{b}^{+}\right)} \mathbb{C}_{\lambda}$ is called the Verma module over $\mathfrak{g}$ with highest weight $\lambda$. Every highest weight $\mathfrak{g}$-module with highest weight $\lambda$ is a homomorphic image of $M(\lambda)$ and the Verma module $M(\lambda)$ contains a unique maximal submodule $J(\lambda)$. Hence the quotient $V(\lambda)=M(\lambda) / J(\lambda)$ is irreducible.

Let $V$ be a $\mathfrak{g}$-module in category $\mathcal{O}$. We define the Casimir operator on $V$ as follows (cf. [24], [30]). Take a linear functional $\rho \in \mathfrak{h}^{*}$ satisfying $\rho\left(h_{i}\right)=\frac{1}{2} a_{i i}$ for all $i \in I$. Such a linear functional is called a Weyl vector of $\mathfrak{g}$. Note that $\left(\rho \mid \alpha_{i}\right)=\rho\left(s_{i} h_{i}\right)=\frac{1}{2}\left(\alpha_{i} \mid \alpha_{i}\right)$ $(i \in I)$. For each positive root $\alpha \in \Phi^{+}$, choose a basis $\left\{e_{\alpha}^{(i)} \mid i=1, \cdots, \operatorname{dim} \mathfrak{g}_{\alpha}\right\}$ of the root space $\mathfrak{g}_{\alpha}$ and let $\left\{e_{-\alpha}^{(i)} \mid i=1, \cdots, \operatorname{dim} \mathfrak{g}_{\alpha}\right\}$ be the dual basis of $\mathfrak{g}_{-\alpha}$ in the sense that $\left(e_{\alpha}^{(i)} \mid e_{-\alpha}^{(j)}\right)=\delta_{i j} \theta(\alpha, \alpha)$. Then for a weight vector $v \in V_{\lambda}$ of weight $\lambda$, the Casimir operator $\Omega$ on $V$ is defined by

$$
\Omega(v)=(\lambda+2 \rho \mid \lambda) v_{\lambda}+2 \sum_{\alpha \in \Phi^{+}} \sum_{i=1}^{\operatorname{dimg} g_{\alpha}} e_{-\alpha}^{(i)} e_{\alpha}^{(i)} v_{\lambda} .
$$

The Casimir operator commutes with the action of $\mathfrak{g}$ on $V$. Recall that a weight vector $v \in V$ is called a primitive vector if there is a $\mathfrak{g}$-submodule $W$ of $V$ such that $v \notin W$ 
and $e_{i k} \cdot v \in W$ for all $i \in I, k=1,2, \cdots, m_{i}$ (cf. [31]). The Casimir operator satisfies the following properties:

Proposition 6.2 (a) If $V$ is a highest weight $\mathfrak{g}$-module in Category $\mathcal{O}$ with highest weight $\Lambda$, then we have

$$
\Omega=(\Lambda+2 \rho \mid \Lambda) I_{V}
$$

(b) If $v$ is a primitive vector with weight $\lambda$, then there exists a $\mathfrak{g}$-submodule $W \subset V$ such that $v \notin W$ and

$$
\Omega(v)=(\lambda+2 \rho \mid \lambda) v \bmod W
$$

Let $P^{+}$be the set of all linear functionals $\lambda \in \mathfrak{h}^{*}$ satisfying

$$
\left\{\begin{array}{l}
\lambda\left(h_{i}\right) \in \mathbb{Z}_{\geq 0} \text { for all } i \in I^{r e} \\
\lambda\left(h_{i}\right) \in 2 \mathbb{Z}_{\geq 0} \text { for all } i \in I^{r e} \cap I^{\text {odd }} \\
\lambda\left(h_{i}\right) \geq 0 \text { for all } i \in I^{i m}
\end{array}\right.
$$

The elements of $P^{+}$are called the dominant integral weights. For a dominant integral weight $\Lambda \in P^{+}$, let

$$
\begin{aligned}
& \Phi^{+}(\Lambda)=\left\{\beta=\sum_{i \in I^{i m}} k_{i} \alpha_{i} \in Q^{+} \mid\left(\Lambda \mid \alpha_{i}\right)=0 \text { for } k_{i} \geq 1\right. \\
& \left.\left(\alpha_{i} \mid \alpha_{j}\right)=0 \text { for } k_{i}, k_{j} \geq 1, i \neq j, \quad\left(\alpha_{i} \mid \alpha_{i}\right)=0 \text { for } k_{i} \geq 2\right\} .
\end{aligned}
$$

For such an element $\beta \in \Phi^{+}(\Lambda)$, we denote $|\beta|=\sum_{i \in I^{i m}} k_{i}$ and

$$
\varepsilon(\beta)=\prod_{i \in I^{i m} \cap I^{\text {even }}}\left(\begin{array}{c}
m_{i} \\
k_{i}
\end{array}\right) \prod_{j \in I^{i m} \cap I^{\text {odd }}}\left(\begin{array}{c}
m_{j}+k_{j}-1 \\
k_{j}
\end{array}\right) .
$$

Then the character of the irreducible highest weight module $V(\Lambda)$ with highest weight $\Lambda \in P^{+}$is determined by the Weyl-Kac-Borcherds formula:

Proposition 6.3 (44], [51])

$$
\operatorname{ch} V(\Lambda)=\frac{\prod_{\alpha \in \Phi_{1}^{-}}\left(1+e^{\alpha}\right)^{d i m \mathfrak{g}_{\alpha}}}{\prod_{\alpha \in \Phi_{0}^{-}}\left(1-e^{\alpha}\right)^{\operatorname{dim} \mathfrak{g}_{\alpha}}} \sum_{\substack{w \in W \\ \beta \in \Phi^{+}(\Lambda)}}(-1)^{l(w)+|\beta|} \varepsilon(\beta) e^{w(\Lambda+\rho-\beta)-\rho} .
$$

Letting $\Lambda=0$, we obtain the denominator identity:

$$
\frac{\prod_{\alpha \in \Phi_{0}^{-}}\left(1-e^{\alpha}\right)^{d i m \mathfrak{g}_{\alpha}}}{\prod_{\alpha \in \Phi_{1}^{-}}\left(1+e^{\alpha}\right)^{\operatorname{dim} \mathfrak{g}_{\alpha}}}=\sum_{\substack{w \in W \\ \beta \in \Phi^{+}(0)}}(-1)^{l(w)+|\beta|} \varepsilon(\beta) e^{w(\rho-\beta)-\rho} .
$$


Corollary 6.4 A highest weight $\mathfrak{g}$-module $V$ with highest weight $\Lambda \in P^{+}$and highest weight vector $v_{\Lambda}$ is irreducible if and only if it satisfies:

$$
\begin{aligned}
& f_{i k}^{\Lambda\left(h_{i}\right)+1} \cdot v_{\Lambda}=0 \text { if } i \in I^{r e}, \\
& f_{i k} \cdot v_{\Lambda}=0\left(k=1, \cdots, m_{i}\right) \text { if } \Lambda\left(h_{i}\right)=0 .
\end{aligned}
$$

Suppose a group $G$ acts on the generalized Kac-Moody superalgebra $\mathfrak{g}=\mathfrak{g}(A, \underline{m}, C)$ by Lie superalgebra automorphisms of $\mathfrak{g}_{ \pm}$preserving the root space decomposition. Assume further that $\operatorname{str}\left(g \mid \mathfrak{g}_{\alpha}\right)=\operatorname{str}\left(g \mid \mathfrak{g}_{-\alpha}\right)$ for all $g \in G$ and $\alpha \in Q^{+}$. We would like to apply our supertrace formula (2.17) to derive a closed form formula for $\operatorname{str}\left(g \mid \mathfrak{g}_{\alpha}\right)$ $(g \in G, \alpha \in Q)$.

Let $J$ be a finite subset of $I^{r e}$, and let $\Phi_{J}=\Phi \cap\left(\sum_{j \in J} \mathbb{Z} \alpha_{j}\right), \Phi_{J}^{ \pm}=\Phi^{ \pm} \cap \Phi_{J}$, and $\Phi^{ \pm}(J)=\Phi^{ \pm} \backslash \Phi_{J}^{ \pm}$. We also denote $Q_{J}=Q \cap\left(\sum_{j \in J} \mathbb{Z} \alpha_{j}\right), Q_{J}^{ \pm}=Q^{ \pm} \cap Q_{J}$, and $Q^{ \pm}(J)=Q^{ \pm} \backslash Q_{J}^{ \pm}$. Let $\mathfrak{g}_{0}^{(J)}=\mathfrak{h} \oplus\left(\bigoplus_{\alpha \in \Phi_{J}} \mathfrak{g}_{\alpha}\right)$, and $\mathfrak{g}_{ \pm}^{(J)}=\bigoplus_{\alpha \in \Phi^{ \pm}(J)} \mathfrak{g}_{\alpha}$. Then we have the triangular decomposition:

$$
\mathfrak{g}=\mathfrak{g}_{-}^{(J)} \oplus \mathfrak{g}_{0}^{(J)} \oplus \mathfrak{g}_{+}^{(J)}
$$

where $\mathfrak{g}_{0}^{(J)}$ is the Kac-Moody superalgebra (with an extended Cartan subalgebra) associated with the generalized Cartan matrix $A_{J}=\left(a_{i j}\right)_{i, j \in J}$ and the set of odd indices $J^{\text {odd }}=J \cap I^{\text {odd }}=\left\{j \in J \mid \theta_{j j}=-1\right\}$, and $\mathfrak{g}_{-}^{(J)}$ (resp. $\left.\mathfrak{g}_{+}^{(J)}\right)$ is a direct sum of irreducible highest weight (resp. lowest weight) modules over $\mathfrak{g}_{0}^{(J)}$ (cf. [30]).

To apply our supertrace formula (2.17) to the Lie superalgebra $\mathfrak{g}_{-}^{(J)}$, we need to compute the generalized supercharacters of the homology modules $H_{k}\left(\mathfrak{g}_{-}^{(J)}\right)$. We conjecture that the $\mathfrak{g}_{0}^{(J)}$-module structure of $H_{k}\left(\mathfrak{g}_{-}^{(J)}\right)$ is determined by Kostant's formula. More precisely, let $W_{J}$ be the subgroup of $W$ generated by the simple reflections $r_{j}$ with $j \in J$, and let $W(J)=\left\{w \in W \mid \Phi_{w} \subset \Phi^{+}(J)\right\}$, where $\Phi_{w}=\left\{\alpha \in \Phi^{+} \mid w^{-1} \alpha<0\right\}$. Thus $W_{J}$ is the Weyl group of the Kac-Moody superalgebra $\mathfrak{g}_{0}^{(J)}$ and $W(J)$ is the set of right coset representatives of $W_{J}$ in $W$. That is, $W=W_{J} W(J)$. Let us denote by $\Phi_{J, i}^{ \pm}=\Phi_{J} \cap \Phi_{i}^{ \pm}(i=0,1)$ and $\Phi_{i}^{ \pm}(J)=\Phi_{i}^{ \pm} \backslash \Phi_{J, i}^{ \pm}(i=0,1)$. Then we have the following conjecture for the structure of $H_{k}\left(\mathfrak{g}_{-}^{(J)}\right)$ :

\section{Conjecture:}

$$
H_{k}\left(\mathfrak{g}_{-}^{(J)}\right) \cong \sum_{\substack{w \in W(J) \\ \beta \in \Phi^{+}(0) \\ l(w)+|\beta|=k}} V_{J}(w(\rho-\beta)-\rho)^{\oplus \varepsilon(\beta)}
$$

where $V_{J}(\mu)$ denotes the irreducible highest weight module over the Kac-Moody superalgebra $\mathfrak{g}_{0}^{(J)}$ with highest weight $\mu$.

Remark. The formula (6.18) was first introduced by Kostant for finite dimensional simple Lie algebras (43]). It was generalized to symmetrizable Kac-Moody algebras 
([17], 44]) and generalized Kac-Moody algebras (49]). But, in general, it is not true for Lie superalgebras. Still, we conjecture that Kostant's formula holds for generalized Kac-Moody superalgebras associated with the Borcherds-Cartan matrix which is colored by a coloring matrix.

For each $k \geq 1$, let

$$
H_{k}^{(J)}=\sum_{\substack{w \in W(J) \\ \beta \in \Phi^{+}(0) \\ l(w)+|\beta|=k}} V_{J}(w(\rho-\beta)-\rho)^{\oplus \varepsilon(\beta)},
$$

and define the homology superspace

$$
H^{(J)}=\sum_{k=1}^{\infty}(-1)^{k+1} H_{k}^{(J)}=H_{1}^{(J)} \ominus H_{2}^{(J)} \oplus H_{3}^{(J)} \ominus \cdots,
$$

an alternating direct sum of superspaces. Then, for all $g \in G$ and $\alpha \in Q_{-}$, we have

$$
\operatorname{str}\left(g \mid H_{\alpha}^{(J)}\right)=\sum_{\substack{w \in W(J) \\ \beta \in \Phi^{+}(0) \\ l(w)+|\beta| \geq 1}}(-1)^{l(w)+|\beta|+1} \varepsilon(\beta) \operatorname{str}\left(g \mid V_{J}(w(\rho-\beta)-\rho)_{\alpha}\right),
$$

and the generalized denominator identity can be written as

$$
\begin{gathered}
\prod_{\alpha \in \Phi^{-}(J)} \exp \left(-\sum_{k=1}^{\infty} \frac{1}{k} \operatorname{str}\left(g^{k} \mid \mathfrak{g}_{\alpha}\right) E^{k \alpha}\right)=1-\operatorname{sch}_{g} H^{(J)} \\
=\sum_{\substack{w \in W(J) \\
\beta \in \Phi^{+}(0)}}(-1)^{l(w)+|\beta|} \varepsilon(\beta) \operatorname{sch}_{g} V_{J}(w(\rho-\beta)-\rho) .
\end{gathered}
$$

Let $P\left(H^{(J)}\right)=\left\{\tau \in Q_{-} \mid \operatorname{str}\left(g \mid H_{\tau}^{(J)}\right) \neq 0\right\}=\left\{\tau_{1}, \tau_{2}, \tau_{3}, \cdots\right\}$, and $t_{g}(i)=\operatorname{str}\left(g \mid H_{\tau_{i}}^{(J)}\right)$. For $\tau \in Q_{-}$, define the set $T^{(J)}(\tau)$ of all partitions of $\tau$ into a sum of $\tau_{i}$ 's as in (2.15) and define the Witt partition function $W_{g}^{(J)}(\tau)$ as in (2.16). Then, our supertrace formula (2.17) yields:

Theorem 6.5 Suppose that a group $G$ acts on the generalized Kac-Moody superalgebra $\mathfrak{g}=\mathfrak{g}(A, \underline{m}, C)$ by Lie superalgebra automorphisms of $\mathfrak{g}_{ \pm}$preserving the root space decomposition. Assume further that $\operatorname{str}\left(g \mid \mathfrak{g}_{\alpha}\right)=\operatorname{str}\left(g \mid \mathfrak{g}_{-\alpha}\right)$ for all $g \in G$ and $\alpha \in Q^{+}$. Let $J$ be a finite subset of $I^{r e}$. Then, for all $g \in G$ and $\alpha \in \Phi^{-}(J)$, we have

$$
\begin{aligned}
& \operatorname{str}\left(g \mid \mathfrak{g}_{\alpha}\right)=\sum_{d \mid \alpha} \frac{1}{d} \mu(d) W_{g^{d}}^{(J)}\left(\frac{\alpha}{d}\right) \\
& =\sum_{d \mid \alpha} \frac{1}{d} \mu(d) \sum_{s \in T^{(J)}(\alpha / d)} \frac{(|s|-1) !}{s !} \prod t_{g^{d}}(i)^{s_{i}},
\end{aligned}
$$


where $\mu$ is the classical Möbius function, and, for a positive integer $d, d \mid \alpha$ denotes $\alpha=d \tau$ for some $\tau \in Q_{-}$, in which case $\alpha / d=\tau$.

We would like to remind the readers that our supertrace formula (6.23) has been derived under the assumption that Kostant's formula holds for generalized Kac-Moody superalgebras. However, in some special cases, we can derive the supertrace formula for generalized Kac-Moody superalgebras wihtout assuming Kostant's formula.

For example, suppose that the Borcherds-Cartan matrix $A$ satisfies: (i) the set $I^{r e}$ is finite, (ii) $a_{i j} \neq 0$ for all $i, j \in I^{i m}$. Then it is known that the Lie superalgebra $\mathfrak{g}_{-}^{(J)}=\bigoplus_{\alpha \in \Delta^{-}(J)} \mathfrak{g}_{\alpha}$ (resp. $\mathfrak{g}_{+}^{(J)}=\bigoplus_{\alpha \in \Delta^{+}(J)} \mathfrak{g}_{\alpha}$ ) is isomorphic to the free Lie superalgebra generated by the superspace $V=\bigoplus_{j \in I^{i m}} V_{J}\left(-\alpha_{j}\right)^{\oplus m_{j}}$ (resp. $V^{*}=\bigoplus_{j \in I^{i m}} V_{J}^{*}\left(-\alpha_{j}\right)^{\oplus m_{j}}$ ), where $V_{J}(\mu)$ (resp. $\left.V_{J}^{*}(\mu)\right)$ denotes the irreducible highest weight (resp. lowest weight) module over the Kac-Moody superalgebra $\mathfrak{g}_{0}^{(J)}$ with highest weight $\mu$ (resp. lowest weight $-\mu$ ) (cf. 40]). Hence, for any $g \in G$, the generalized denominator identity of the Lie superalgebra $\mathfrak{g}_{-}^{(J)}$ is the same as

$$
\begin{gathered}
\prod_{\alpha \in \Phi^{-}(J)} \exp \left(-\sum_{k=1}^{\infty} \frac{1}{k} \operatorname{str}\left(g^{k} \mid \mathfrak{g}_{\alpha}\right) E^{k \alpha}\right)=1-\operatorname{sch}_{g} V \\
=1-\sum_{j \in I^{i m}} m_{j} \operatorname{sch}_{g} V_{J}\left(-\alpha_{j}\right) .
\end{gathered}
$$

Hence, by our supertrace formula (2.17), we obtain

$$
\operatorname{str}\left(g \mid \mathfrak{g}_{\alpha}\right)=\sum_{d \mid \alpha} \frac{1}{d} \mu(d) \sum_{s \in T^{(J)}(\alpha / d)} \frac{(|s|-1) !}{s !} \prod t_{g^{d}}(i)^{s_{i}}
$$

where $t_{g}(i)=\operatorname{str}\left(g \mid V_{\tau_{i}}\right)=\sum_{j \in I^{i m}} m_{j} \operatorname{str}\left(g \mid V_{J}\left(-\alpha_{j}\right)_{\tau_{i}}\right)$ for $i=1,2,3, \cdots$.

\section{Monstrous Lie Superalgebras}

Let $F(q)=\sum_{n=-1}^{\infty} f(n) q^{n}=q^{-1}+f(1) q+f(2) q^{2}+\cdots$ be a normalized $q$-series such that $f(-1)=1, f(0)=0$, and $f(n) \in \mathbb{Z}$ for all $n \geq 1$. We say that $F(q)$ is convergent if it converges for all $q=e^{2 \pi i \tau}, \operatorname{Im} \tau>0$. Observe that, for each $m \geq 1$, there exists a unique polynomial $P_{m}(t) \in \mathbb{Z}[t]$ such that

$$
P_{m}(F) \equiv q^{-m} \bmod q \mathbb{Z}[[q]]
$$

A convergent normalized $q$-series $F(q)=\sum_{n=-1}^{\infty} f(n) q^{n}=q^{-1}+f(1) q+f(2) q^{2}+\cdots$ is called replicable if for all $m>0$ and for all $a \mid m$, there exist normalized $q$-series 
$F^{(a)}(q)=\sum_{n=-1}^{\infty} f^{(a)}(n) q^{n}=q^{-1}+f^{(a)}(1) q+f^{(a)}(2) q^{2}+\cdots$ with $f^{(a)}(-1)=1$ $f^{(a)}(0)=0$, and $f^{(a)}(n) \in \mathbb{Z}$ for all $n \geq 1$ such that

$$
F^{(1)}=F, \text { and } \sum_{\substack{a d=m \\ 0 \leq b<d}} F^{(a)}\left(\frac{a \tau+b}{d}\right)=P_{m}(F),
$$

where $q=e^{2 \pi i \tau}, \operatorname{Im} \tau>0$.

In [40], the replicable functions were characterized in terms of product identities. More precisely, a normalized $q$-series $F(q)=q^{-1}+f(1) q+f(2) q^{2}+\cdots=\sum_{n=-1}^{\infty} f(n) q^{n}$ is replicable if and only if for all $k \geq 1$, there exist normalized $q$-series $F^{(k)}(q)=$ $\sum_{n=-1}^{\infty} f^{(k)}(n) q^{n}$ satisfying the product identity

$$
\prod_{m, n=1}^{\infty} \exp \left(-\sum_{k=1}^{\infty} \frac{1}{k} f^{(k)}(m n) p^{k m} q^{k n}\right)=1-\sum_{i, j=1}^{\infty} f(i+j-1) p^{i} q^{j}
$$

Now, we will consider the generalized Kac-Moody superalgebras associated with the normalized $q$-series. We take $I=\{-1\} \cup\{1,2,3, \cdots\}$ to be the index set, and let $A=(-(i+j))_{i, j \in I}$ be the Borcherds-Cartan matrix of the Monster Lie algebra ([6]). Consider a normalized $q$-series $F(q)=\sum_{n=-1}^{\infty} f(n) q^{n}$ such that $f(-1)=1$, $f(0)=0$, and $f(n) \in \mathbb{Z}$ for all $n \geq 1$. We define the charge of the matrix $A$ to be $\underline{m}=(|f(i)|: i \in I)$, and choose a coloring matrix $C=\left(\theta_{i j}\right)_{i, j \in I}$ such that $\theta_{i i}=1$ if $f(i)>0$ and $\theta_{i i}=-1$ if $f(i)<0$. That is, an index $i \in I$ is even if $f(i)>0$ and odd if $f(i)<0$.

The generalized Kac-Moody superalgebra $\mathfrak{L}(F)=\mathfrak{g}(A, \underline{m}, C)$ associated with the above data is called the Monstrous Lie superalgebra associated with the normalized $q$-series $F(q)=\sum_{n=-1}^{\infty} f(n) q^{n}$. For example, the Monstrous Lie superalgebra associated with the elliptic modular function $J(q)=j(q)-744$ is the Monster Lie algebra, and the Monstrous Lie superalgebras associated with the Thompson series $T_{g}(q)=\sum_{n=-1}^{\infty} c_{g}(n) q^{n}$ are the Monstrous Lie superalgebras given in [6].

Let $\mathfrak{L}(F)$ be the Monstrous Lie superalgebra associated with a normalized $q$-series $F(q)=\sum_{n=-1}^{\infty} f(n) q^{n}$. By our choice of the charge and the coloring matrix, we see that $\alpha_{-1}$ is the only real even simple root, $\alpha_{i}(i \geq 1)$ is an imaginary even simple root with multiplicity $f(i)$ if $f(i)>0$, and an imaginary odd simple root with multiplicity $-f(i)$ if $f(i)<0$. If we identify the simple roots $\alpha_{-1}$ with $(1,-1) \in I I_{1,1}$ and $\alpha_{i}$ with $(1, i) \in I I_{1,1}(i \geq 1)$, then the Monstrous Lie superalgebra $\mathfrak{L}(F)$ becomes a $I I_{1,1}$-graded Lie superalgebra, and we have

$$
\mathfrak{L}(F)_{+}=\bigoplus_{\substack{m>0 \\ n \in \mathbf{Z}}} \mathfrak{L}(F)_{(m, n)}, \quad \mathfrak{L}(F)_{-}=\bigoplus_{\substack{m>0 \\ n \in \mathbf{Z}}} \mathfrak{L}(F)_{(-m, n)} .
$$

Suppose that a group $G$ acts on $\mathfrak{L}(F)$ by Lie superalgebra automorphisms of $\mathfrak{L}(F)_{ \pm}$preserving the $I I_{1,1}$-gradation. Assume further that $\operatorname{str}\left(g \mid \mathfrak{L}(F)_{(m, n)}\right)=\operatorname{str}\left(g \mid \mathfrak{L}(F)_{(-m,-n)}\right)$ 
for all $g \in G, m, n>0$, and

$$
g \cdot f_{-1}=f_{-1}, \quad g \cdot e_{-1}=e_{-1} \text { for all } g \in G .
$$

We will apply our supertrace formula (6.23) (actually (6.25) ) to this setting.

Let $V_{-1}=\mathfrak{L}_{(-1,1)}=\mathbb{C} f_{-1}, V_{i}=\mathfrak{L}(F)_{(-1,-i)}=\mathbb{C} f_{i, 1} \oplus \cdots \oplus \mathbb{C} f_{i,|f(i)|}$ for $i \geq 1$, and $V=\bigoplus_{n=-1}^{\infty} V_{n}$. For $g \in G$ and $i \in I=\{-1\} \cup\{1,2,3, \cdots\}$, define $f_{g}(i)=\operatorname{str}\left(g \mid V_{i}\right)$. Then $V=\bigoplus_{n=-1}^{\infty} V_{n}$ is a $\mathbb{Z}$-graded representation of the group $G$ such that $\operatorname{sch}(V)=$ $F(q)=\sum_{n=-1}^{\infty} f(n) q^{n}$ and $\operatorname{sch}_{g}(V)=F_{g}(q)=\sum_{n=-1}^{\infty} f_{g}(n) q^{n}$.

Consider a basis of $V_{i}$ consisting of the eigenvectors $v_{i, k}$ of $g \in G$ with eigenvalues $\lambda_{i, k}(k=1, \cdots,|f(i)|)$. Then we have

$$
f_{g}(i)=\operatorname{str}\left(g \mid V_{i}\right)=\psi(1, i) \sum_{k=1}^{|f(i)|} \lambda_{i, k} .
$$

To apply our supertrace formula (6.23), take $J=\{-1\}$ and consider the corresponding triangular decomposition

$$
\mathfrak{L}(F)=\mathfrak{L}(F)_{-}^{(J)} \oplus \mathfrak{L}(F)_{0}^{(J)} \oplus \mathfrak{L}(F)_{+}^{(J)} .
$$

Then $\mathfrak{L}(F)_{0}^{(J)}=\left\langle e_{-1}, f_{-1}, h_{-1}\right\rangle+\mathfrak{h} \cong s l(2, \mathbb{C})+\mathfrak{h}$, and the subalgebra $\mathfrak{L}(F)_{-}^{(J)}$ (resp. $\left.\mathfrak{L}(F)_{+}^{(J)}\right)$ is isomorphic to the free Lie superalgebra generated by the superspace $H^{(J)}=$ $\bigoplus_{i=1}^{\infty} V_{J}\left(-\alpha_{i}\right)^{\oplus|f(i)|}$ (resp. $\left.H^{(J) *}=\bigoplus_{i=1}^{\infty} V_{J}^{*}\left(-\alpha_{i}\right)^{\oplus|f(i)|}\right)$, where $V_{J}\left(-\alpha_{i}\right)$ and $V_{J}^{*}\left(-\alpha_{i}\right)$ are the $i$-dimensional irreducible representations of $\operatorname{sl}(2, \mathbb{C})\left(\right.$ since $\left.-\alpha_{i}\left(h_{-1}\right)=i-1\right)$. We will concentrate on the Lie superalgebra $\mathfrak{L}(F)_{-}^{(J)}$.

Since the weights of $V_{J}\left(-\alpha_{i}\right)$ are $-\alpha_{i}=(-1,-i),-\alpha_{i}-\alpha_{-1}=(-2,-i+1), \cdots$, $-\alpha_{i}-(i-1) \alpha_{-1}=(-i,-1)$, we have

$$
P\left(H^{(J)}\right)=\left\{(-i,-j) \mid i, j \in \mathbb{Z}_{>0}\right\} .
$$

Note that each $f_{i, k}$ generates an $i$-dimensional irreducible representation of the Lie algebra $\operatorname{sl}(2, \mathbb{C})$ generated by $e_{-1}, f_{-1}, h_{-1}$, and hence so does each $v_{i, k}(i \geq 1, k=$ $1,2, \cdots,|f(i)|)$. Thus we have

$$
\begin{aligned}
& H_{(-i,-j)}^{(J)}=\bigoplus_{k=1}^{|f(i+j-1)|} \mathbb{C}\left(a d f_{-1}\right)^{i-1}\left(f_{i+j-1, k}\right) \\
& =\bigoplus_{k=1}^{|f(i+j-1)|} \mathbb{C}\left(a d f_{-1}\right)^{i-1}\left(v_{i+j-1, k}\right) .
\end{aligned}
$$

It follows that

$$
\begin{aligned}
g \cdot\left(a d f_{-1}\right)^{i-1}\left(v_{i+j-1, k}\right) & =\left(a d\left(g \cdot f_{-1}\right)\right)^{i-1}\left(g \cdot v_{i+j-1, k}\right) \\
& =\lambda_{i+j-1, k}\left(a d f_{-1}\right)^{i-1}\left(v_{i+j-1, k}\right)
\end{aligned}
$$


which yields

$$
\operatorname{str}\left(g \mid H_{(-i,-j)}^{(J)}\right)=\psi(1, i+j-1) \sum_{k=1}^{|f(i+j-1)|} \lambda_{i+j-1, k}=f_{g}(i+j-1)
$$

for all $g \in G, i, j \in \mathbb{Z}_{>0}$. Hence the generalized denominator identity for $g \in G$ of the Lie superalgebra $\mathfrak{L}(F)_{-}^{(J)}$ is equal to

$$
\begin{gathered}
\prod_{m, n=1} \exp \left(-\sum_{k=1}^{\infty} \frac{1}{k} \operatorname{str}\left(g^{k} \mid \mathfrak{L}(F)_{(-m,-n)}^{(J)}\right) p^{k m} q^{k n}\right) \\
=1-\sum_{i, j=1}^{\infty} f_{g}(i+j-1) p^{i} q^{j} .
\end{gathered}
$$

For $k, l>0$, we have

$$
T^{(J)}(k, l)=T(k, l)=\left\{s=\left(s_{i j}\right)_{i, j \geq 1} \mid s_{i j} \in \mathbf{Z}_{\geq 0}, \sum s_{i j}(i, j)=(k, l)\right\},
$$

the set of all partitions of $(k, l)$ into a sum of ordered pairs of positive integers, and the Witt partition function $W_{g}^{(J)}(k, l)$ is given by

$$
\begin{aligned}
W_{g}^{(J)}(k, l) & =\sum_{s \in T(k, l)} \frac{(|s|-1) !}{s !} \prod \operatorname{str}\left(g \mid H_{(-i,-j)}^{(J)}\right)^{s_{i j}} \\
& =\sum_{s \in T(k, l)} \frac{(|s|-1) !}{s !} \prod f_{g}(i+j-1)^{s_{i j}}
\end{aligned}
$$

Therefore, our supertrace formula (6.23) yields:

Proposition 7.1 Let $\mathfrak{L}(F)=\bigoplus_{(m, n) \in I I_{1,1}} \mathfrak{L}(F)_{(m, n)}$ be the Monstrous Lie superalgebra associated with a normalized q-series $F(q)=\sum_{n=-1}^{\infty} f(n) q^{n}$ and let $V=\bigoplus_{n=-1}^{\infty} V_{n}=$ $\bigoplus_{n=-1}^{\infty} \mathfrak{L}(F)_{(-1,-n)}$. Suppose that a group $G$ acts on $\mathfrak{L}(F)$ by Lie superalgebra automorphisms of $\mathfrak{L}(F)_{ \pm}$preserving the $I_{1,1}$-gradation. Assume further that $\operatorname{str}\left(g \mid \mathfrak{L}(F)_{(m, n)}\right)=$ $\operatorname{str}\left(g \mid \mathfrak{L}(F)_{(-m,-n)}\right)$ for all $g \in G, m, n>0$, and

$$
g \cdot f_{-1}=f_{-1}, \quad g \cdot e_{-1}=e_{-1} \quad \text { for all } g \in G .
$$

Then, for all $g \in G$ and $m, n>0$, we have

$$
\begin{aligned}
& \operatorname{str}\left(g \mid \mathfrak{L}(F)_{(m, n)}\right)=\operatorname{str}\left(g \mid \mathfrak{L}(F)_{(-m,-n)}\right) \\
& =\sum_{d \mid(m, n)} \frac{1}{d} \mu(d) \sum_{s \in T\left(\frac{m}{d}, \frac{n}{d}\right)} \frac{(|s|-1) !}{s !} \prod f_{g^{d}}(i+j-1)^{s_{i j}},
\end{aligned}
$$

where $f_{g}(i)=\operatorname{str}\left(g \mid V_{i}\right)=\operatorname{str}\left(g \mid \mathfrak{L}_{(-1,-i)}\right)$ for all $g \in G$ and $i=1,2,3, \cdots$. 
Corollary 7.2 Suppose that the generalized supercharacters $F_{g}(q)=\sum_{n=-1}^{\infty} f_{g}(n) q^{n}$ are replicable with the replicates $F_{g}^{(a)}(a \geq 1)$ for all $g \in G$. Then we have

$$
\operatorname{str}\left(g \mid \mathfrak{L}(F)_{(m, n)}\right)=\operatorname{str}\left(g \mid \mathfrak{L}(F)_{(-m,-n)}\right)=\sum_{a d \mid(m, n)} \frac{1}{a d} \mu(d) f_{g^{d}}^{(a)}\left(\frac{m n}{a^{2} d^{2}}\right) .
$$

Proof. Since the generalized supercharacter $F_{g}(q)$ is replicable, by (7.1), the generalized denominator identity for $g \in G$ of the Lie superalgebra $\mathfrak{L}(F)_{-}^{(J)}$ is equal to

$$
\begin{aligned}
\prod_{m, n=1}^{\infty} \exp & \left(-\sum_{k=1}^{\infty} \frac{1}{k} \operatorname{str}\left(g^{k} \mid \mathfrak{L}(F)_{(-m,-n)}\right) p^{k m} q^{k n}\right) \\
= & 1-\sum_{i, j=1}^{\infty} f_{g}(i+j-1) p^{i} q^{j} \\
& =\prod_{m, n=1}^{\infty} \exp \left(-\sum_{a=1}^{\infty} \frac{1}{a} f_{g}^{(a)}(m n) p^{a m} q^{a n}\right) .
\end{aligned}
$$

By taking the logarithm, we have

$$
\begin{aligned}
\log \prod_{m, n=1}^{\infty} \exp & \left(-\sum_{k=1}^{\infty} \frac{1}{k} \operatorname{str}\left(g^{k} \mid \mathfrak{L}(F)_{(-m,-n)}\right) p^{k m} q^{k n}\right) \\
= & -\sum_{m, n=1}^{\infty} \sum_{k=1}^{\infty} \frac{1}{k} \operatorname{str}\left(g^{k} \mid \mathfrak{L}(F)_{(-m,-n)}\right) p^{k m} q^{k n} \\
= & -\sum_{m, n=1}^{\infty} \sum_{\substack{k>0 \\
k \mid(m, n)}} \frac{1}{k} \operatorname{str}\left(g^{k} \mid \mathfrak{L}(F)_{\left(-\frac{m}{k},-\frac{n}{k}\right)}\right) p^{m} q^{n}
\end{aligned}
$$

and

$$
\begin{aligned}
\log \prod_{m, n=1}^{\infty} \exp & \left(-\sum_{a=1}^{\infty} \frac{1}{a} f_{g}^{(a)}(m n) p^{a m} q^{a n}\right) \\
& =-\sum_{m, n=1}^{\infty} \sum_{a=1}^{\infty} \frac{1}{a} f_{g}^{(a)}(m n) p^{a m} q^{a n} \\
& =-\sum_{m, n=1}^{\infty} \sum_{\substack{a>0 \\
a \mid(m, n)}} \frac{1}{a} f_{g}^{(a)}\left(\frac{m n}{a^{2}}\right) p^{m} q^{n}
\end{aligned}
$$

It follows that

$$
\sum_{\substack{k>0 \\ k \mid(m, n)}} \frac{1}{k} \operatorname{str}\left(g^{k} \mid \mathfrak{L}(F)_{\left(-\frac{m}{k},-\frac{n}{k}\right)}\right)=\sum_{\substack{a>0 \\ a \mid(m, n)}} \frac{1}{a} f_{g}^{(a)}\left(\frac{m n}{a^{2}}\right) .
$$

Therefore, by Möbius inversion, we obtain

$$
\operatorname{str}\left(g \mid \mathfrak{L}(F)_{(-m,-n)}\right)=\sum_{a d \mid(m, n)} \frac{1}{a d} \mu(d) f_{g^{d}}^{(a)}\left(\frac{m n}{a^{2} d^{2}}\right) .
$$




\section{Orbit Lie Superalgebras for the Dynkin Diagram Automorphisms of Borcherds-Cartan Data}

In the following two sections, we will discuss the applications of the generalized denominator identities for Dynkin diagram automorphisms of generalized Kac-Moody superalgebras. The notion of orbit Lie superalgebras will play an important role in our discussion. Most of our result is a generalization of the corresonding result on Kac-Moody algebras and generalized Kac-Moody algebras obtained in [13] and [14]. Let $A=\left(a_{i j}\right)_{i, j \in I}$ be a Borcherds-Cartan matrix of charge $\underline{m}=\left(m_{i} \mid i \in I\right)$ with a coloring matrix $C=\left(\theta_{i j}\right)_{i, j \in I}$, and let $\mathfrak{g}=\mathfrak{g}(A, \underline{m}, C)$ be the corresponding generalized Kac-Moody superalgebra.

Definition 8.1 A bijection $\sigma: I \rightarrow I$ is called a Dynkin diagram automorphism of the Borcherds-Cartan data $(A, \underline{m}, C)$ if it satisfies: (i) $a_{i j}=a_{\sigma(i), \sigma(j)}$ for all $i, j \in I$, (ii) $\sigma\left(I^{\text {even }}\right) \subset I^{\text {even }}, \quad \sigma\left(I^{\text {odd }}\right) \subset I^{\text {odd }}$, (iii) $m_{\sigma(i)}=m_{i}$ for all $i \in I$.

In this paper, we will assume that the Dynkin diagram automorphism has finite order $N$.

Given a Dynkin diagram automorphism $\sigma$ of a Borcherds-Cartan data $(A, \underline{m}, C)$, we extend it to a Lie superalgebra automorphism of $\mathfrak{g}=\mathfrak{g}(A, \underline{m}, C)$ by defining

$$
\begin{aligned}
& \sigma\left(e_{i k}\right)=e_{\sigma(i), k} \text { for } i \in I, 1 \leq k \leq m_{i} \\
& \sigma\left(f_{i k}\right)=f_{\sigma(i), k} \text { for } i \in I, 1 \leq k \leq m_{i} \\
& \sigma\left(h_{i}\right)=h_{\sigma(i)}, \quad \sigma\left(d_{i}\right)=d_{\sigma(i)} \text { for } i \in I .
\end{aligned}
$$

It is straightforward to check that $\sigma$ is indeed a Lie superalgebra automorphism of $\mathfrak{g}$. Let $U=U(\mathfrak{g})$ be the universal enveloping algebra of $\mathfrak{g}$. Then the Dynkin diagram automorphism $\sigma$ of $\mathfrak{g}$ can be extended to an algebra automorphism of $U(\mathfrak{g})$ by

$$
\sigma\left(x_{1} \cdots x_{k}\right)=\sigma\left(x_{1}\right) \cdots \sigma\left(x_{k}\right) \text { for } x_{i} \in \mathfrak{g},
$$

which we will also denote by $\sigma$.

Moreover, the Dynkin diagram automorphism $\sigma$ induces an automorphism $\sigma^{*}$ of $\mathfrak{h}^{*}$ defined by

$$
\sigma^{*}(\lambda)(h)=\lambda\left(\sigma^{-1}(h)\right) \text { for } \lambda \in \mathfrak{h}^{*}, h \in \mathfrak{h} .
$$

In particular, for all $i \in I$, we have $\sigma^{*}\left(\alpha_{i}\right)=\alpha_{\sigma(i)}$. Since $\sigma$ has order $N, \sigma^{*}$ also has order $N$, and we have an eigenspace decomposition

$$
\mathfrak{h}^{*}=\bigoplus_{k=0}^{N-1}\left(\mathfrak{h}^{*}\right)^{(k)}
$$


where $\left(\mathfrak{h}^{*}\right)^{(k)}=\left\{\lambda \in \mathfrak{h}^{*} \mid \sigma^{*}(\lambda)=e^{\frac{2 \pi i k}{N}} \lambda\right\}$. The elements of $\left(\mathfrak{h}^{*}\right)^{(0)}=\left\{\lambda \in \mathfrak{h}^{*} \mid \sigma^{*}(\lambda)=\right.$ $\lambda\}$ are called symmetric.

Now, we will construct a generalized Kac-Moody superalgebra $\breve{\mathfrak{g}}=\mathfrak{g}(\sigma)$, called the orbit Lie superalgebra, associated with the Dynkin diagram automorphism $\sigma$ of $\mathfrak{g}$. Let $\sigma$ be a Dynkin diagram automorphism of the Borcherds-Cartan data $(A, \underline{m}, C)$. Since $\sigma$ permutes the elements of $I, I$ is decomposed into a disjoint union of $\sigma$-orbits: $I=\bigsqcup[i]$, where $[i]=\left\{\sigma^{k}(i) \mid k=0,1, \cdots, N_{i}-1\right\}$. Let $N_{i}=|[i]|$ denote the number of elements in the $\sigma$-orbit $[i]$. For each $i \in I$, let $\bar{i}$ be the smallest element of the $\sigma$-orbit of $i$, and let $\widehat{I}=\{\bar{i} \mid i \in I\}$ be the set of such representatives. Set $\widehat{I}^{r e}=\widehat{I} \cap I^{r e}, \widehat{I}^{i m}=\widehat{I} \cap I^{i m}$, $\widehat{I}^{\text {even }}=\widehat{I} \cap I^{\text {even }}$, and $\widehat{I}^{\text {odd }}=\widehat{I} \cap I^{\text {odd }}$.

Let $\widehat{A}=\widehat{A}(\sigma)=\left(\widehat{a}_{\bar{i}, \bar{j}}\right)_{\bar{i}, \bar{j} \in \widehat{I}}$ be the square matrix indexed by $\widehat{I}$ whose entries are defined by

$$
\widehat{a}_{\bar{i}, \bar{j}}=\epsilon_{j} \sum_{k=0}^{N_{j}-1} a_{i, \sigma^{k}(j)} \text { for } \bar{i}, \bar{j} \in \widehat{I}
$$

where $\epsilon_{j}=1-\sum_{k=1}^{N_{j}-1} a_{j, \sigma^{k}(j)}$. Note that $\epsilon_{j}=\epsilon_{\sigma(j)}$ for all $j \in I$.

Let

$$
\breve{I}=\left\{i \in \widehat{I} \mid \epsilon_{i} \leq 2 \text { for } i \in I^{r e}, \epsilon_{i}=1 \text { for } i \in I^{i m}\right\} \text {. }
$$

Since $\widehat{a}_{\bar{i}, \bar{i}}=\epsilon_{i}\left(a_{i i}+1-\epsilon_{i}\right)$, the condition on $\breve{I}$ is equivalent to $\widehat{a}_{\bar{i}, \bar{i}}=a_{i i}$ for all $\bar{i} \in \widehat{I}$. (The first half of the condition (8.4) was introduced in 13 and was referred to as the linking condition.) Also, we set $\breve{A}=\breve{A}(\sigma)=\left(\widehat{a}_{\bar{i}, \bar{j}}\right)_{\bar{i}, \bar{j} \in \breve{I}}, \breve{I}^{r e}=\breve{I} \cap I^{r e}, \breve{I}^{i m}=\breve{I} \cap I^{i m}$, $\breve{I}^{\text {even }}=\breve{I} \cap I^{\text {even }}$, and $\breve{I}^{\text {odd }}=\breve{I} \cap I^{\text {odd }}$.

One can easily verify that $\widehat{A}$ is a symmetrizable Borcherds-Cartan matrix. For instance, let $\widehat{s}_{\bar{i}}=N_{i} \epsilon_{i} s_{i}(\bar{i} \in \widehat{I})$ and let $\widehat{D}=\operatorname{diag}\left(\widehat{s}_{\bar{i}} \mid \bar{i} \in \widehat{I}\right)$, then $\widehat{D} \widehat{A}$ is symmetric.

We define the charge $\underline{\widehat{m}}=\left(\widehat{m}_{\bar{i}} \mid \bar{i} \in \widehat{I}\right)$ and the coloring matrix $\widehat{C}=\left(\widehat{\theta}_{\bar{i}, \bar{j}}\right)_{\bar{i}, \bar{j} \in \widehat{I}}$ of $\widehat{A}$ by

$$
\widehat{m}_{\bar{i}}=m_{i}, \quad \widehat{\theta}_{\bar{i}, \bar{j}}=\theta_{i j} \text { for } \bar{i}, \bar{j} \in \widehat{I} \text {. }
$$

Consider the generalized Kac-Moody superalgebra $\widehat{\mathfrak{g}}=\mathfrak{g}(\widehat{A}, \widehat{m}, \widehat{C})$ associated with the Borcherds-Cartan data $(\widehat{A}, \underline{\widehat{m}}, \widehat{C})$. We denote by $\widehat{e}_{\bar{i}, k}, \widehat{f}_{\bar{i}, k}, \widehat{h}_{\bar{i}}, \widehat{d}_{\bar{i}}\left(\bar{i} \in \widehat{I}, k=1,2, \cdots, m_{i}\right)$ the generators of Lie superalgebra $\widehat{\mathfrak{g}}$, and by $\widehat{\mathfrak{h}}=\left(\bigoplus_{\bar{i} \in \widehat{I}} \mathbb{C} \widehat{h}_{\bar{i}}\right) \oplus\left(\bigoplus_{\bar{i} \in \hat{I}} \mathbb{C} \widehat{d}_{\bar{i}}\right)$ the Cartan subalgebra of $\widehat{\mathfrak{g}}$. We also denote by $\widehat{\Pi}=\left\{\widehat{\alpha}_{\bar{i}} \mid \bar{i} \in \widehat{I}\right\}$ the set of simple roots of $\widehat{\mathfrak{g}}$ and by $\widehat{\Phi}$ the set of roots of $\widehat{\mathfrak{g}}$. The orbit Lie superalgebra $\breve{\mathfrak{g}}=\mathfrak{g}(\sigma)$ is defined to be the subalgebra of $\widehat{\mathfrak{g}}$ generated by $\widehat{e}_{\bar{i}, k}, \widehat{f}_{\bar{i}, k},\left(\bar{i} \in \breve{I}, k=1,2, \cdots, m_{i}\right) \widehat{h}_{\bar{i}}, \widehat{d}_{\bar{i}}\left(\bar{i} \in \widehat{I}, k=1,2, \cdots, m_{i}\right)$. We denote by $\breve{\Pi}=\left\{\widehat{\alpha}_{\bar{i}} \mid \bar{i} \in \widehat{I}\right\}$ the set of simple roots of $\breve{\mathfrak{g}}$ and by $\breve{\Phi}$ the set of roots of $\breve{\mathfrak{g}}$.

As in the case of $\mathfrak{g}=\mathfrak{g}(A, \underline{m}, C)$, there is a symmetric bilinear form on $\widehat{\mathfrak{h}}^{*}$ satisfying $\left(\widehat{\lambda} \mid \widehat{\alpha_{\bar{i}}}\right)=\widehat{\lambda}\left(\widehat{s}_{\bar{i}} \widehat{h}_{\bar{i}}\right)$ for $\widehat{\lambda} \in \widehat{\mathfrak{h}}^{*}, \bar{i} \in \widehat{I}$ (see $\left.(6.2)\right)$. Define a $\mathbb{C}$-linear isomorphism $\phi$ : 
$\left(\mathfrak{h}^{*}\right)^{(0)} \rightarrow \widehat{\mathfrak{h}}^{*}$ by

$$
\begin{aligned}
& \phi(\lambda)\left(\widehat{h}_{\bar{i}}\right)=\lambda\left(h_{i}\right), \\
& \phi(\lambda)\left(\widehat{d}_{\bar{i}}\right)=\epsilon_{i}^{-1} \lambda\left(d_{i}\right) \text { for } \lambda \in\left(\mathfrak{h}^{*}\right)^{(0)}, \bar{i} \in \widehat{I} .
\end{aligned}
$$

Then we obtain the following lemma which will be used in the next section.

Lemma 8.2 For $\lambda, \mu \in\left(\mathfrak{h}^{*}\right)^{(0)}$, we have

$$
(\lambda \mid \mu)=(\phi(\lambda) \mid \phi(\mu))
$$

Equivalently, for $\widehat{\lambda}, \widehat{\mu} \in \widehat{\mathfrak{h}}^{*}$, we have

$$
(\widehat{\lambda} \mid \widehat{\mu})=\left(\phi^{-1}(\widehat{\lambda}) \mid \phi^{-1}(\widehat{\mu})\right)
$$

Proof. First, observe that, $\phi^{-1}\left(\widehat{\alpha}_{\bar{i}}\right)=\epsilon_{i} \sum_{k=0}^{N_{i}-1} \alpha_{\sigma^{k}(i)}$ for $\bar{i} \in \widehat{I}$. Hence for $\widehat{\lambda} \in \widehat{\mathfrak{h}}^{*}$, we obtain

$$
\left(\widehat{\lambda} \mid \widehat{\alpha}_{\bar{i}}\right)=\widehat{\lambda}\left(\widehat{s_{i}} \widehat{h}_{\bar{i}}\right)=\widehat{s_{i}} \widehat{\lambda}\left(\widehat{h}_{\bar{i}}\right)
$$

and

$$
\begin{aligned}
\left(\phi^{-1}(\widehat{\lambda}) \mid \phi^{-1}\left(\widehat{\alpha_{\bar{i}}}\right)\right) & =\left(\phi^{-1}(\widehat{\lambda}) \mid \epsilon_{i} \sum_{k=0}^{N_{i}-1} \alpha_{\sigma^{k}(i)}\right)=\epsilon_{i} \sum_{k=0}^{N_{i}-1}\left(\phi^{-1}(\widehat{\lambda}) \mid \alpha_{\sigma^{k}(i)}\right) \\
& =\epsilon_{i} \sum_{k=0}^{N_{i}-1} \phi^{-1}(\widehat{\lambda})\left(s_{\sigma^{k}(i)} h_{\sigma^{k}(i)}\right)=\epsilon_{i} N_{i} \phi^{-1}(\widehat{\lambda})\left(s_{i} h_{i}\right) \\
& =\epsilon_{i} N_{i} s_{i} \phi^{-1}(\widehat{\lambda})\left(h_{i}\right)=s_{i} \epsilon_{i} N_{i} \widehat{\lambda}\left(\widehat{h_{\bar{i}}}\right)
\end{aligned}
$$

which proves our claim.

Observe that if $\epsilon_{i}=1$, the $\sigma$-orbit of $i$ in the Dynkin diagram of $\mathfrak{g}$ is the disjoint union of $N_{i}$ copies of $A_{1}$ diagram or $B(0,1)$ diagram. If $\epsilon_{i}=2$, then $N_{i}$ is even and the $\sigma$-orbit of $i$ in the Dynkin diagram of $\mathfrak{g}$ is the disjoint union of $N_{i} / 2$ copies of $A_{2}$ diagram.

Next, we will show that the Weyl group $\breve{W}$ of $\breve{\mathfrak{g}}$ is isomorphic to a subgroup of $W$. We may assume that $\breve{W}$ acts on $\widehat{\mathfrak{h}}^{*}$ since $\breve{\mathfrak{g}}$ is a subalgebra of $\widehat{\mathfrak{g}}$. For each $\bar{i} \in \breve{I}^{\text {re }}$, we denote by $\breve{w}_{i}$ the simple reflection in $\breve{W}$ and define

$$
\bar{w}_{\bar{i}}= \begin{cases}\prod_{k=0}^{N_{i}-1} w_{\sigma^{k}(i)} & \text { if } \epsilon_{i}=1 \\ \prod_{l=0}^{N_{i} / 2-1} w_{\sigma^{l}(i)} w_{\sigma^{l+N_{i} / 2}(i)} w_{\sigma^{l}(i)} & \text { if } \epsilon_{i}=2\end{cases}
$$

where $w_{i}$ is simple reflection in $W\left(i \in I^{r e}\right)$. Since $a_{i, \sigma^{k}(i)}=0$ for all $k \not \equiv 0\left(\bmod N_{i}\right)$ if $\epsilon_{i}=1$ and $a_{i, \sigma^{N_{i} / 2}(i)}=-1$ if $\epsilon_{i}=2, \bar{w}_{\bar{i}}$ is well-defined. Let $\bar{W}$ be the subgroup of $W$ generated by $\bar{w}_{i}^{\prime}$ 's $\left(\bar{i} \in \breve{I}^{r e}\right)$. 
Proposition 8.3 For all $\bar{i} \in \breve{I}^{r e}$, we have:

(a) $\bar{w}_{\bar{i}}^{2}=1$.

(b) $\sigma^{*} \bar{w}_{\bar{i}}=\bar{w}_{\bar{i}} \sigma^{*}$. In particular, $\left(\mathfrak{h}^{*}\right)^{(0)}$ is invariant under $\bar{w}_{\bar{i}}$.

(c) For each $\lambda \in\left(\mathfrak{h}^{*}\right)^{(0)}$, we have

$$
\bar{w}_{\bar{i}}(\lambda)=\lambda-\epsilon_{i} \lambda\left(h_{i}\right) \sum_{k=0}^{N_{i}-1} \alpha_{\sigma^{k}(i)} .
$$

(d) For each simple reflection $\breve{w}_{\bar{i}} \in \breve{W}$, we have $\bar{w}_{\bar{i}}=\phi^{-1} \breve{w}_{\bar{i}} \phi$.

Proof. The proofs of (a), (b) and (c) are straightforward.

For $(\mathrm{d})$, let $\lambda \in\left(\mathfrak{h}^{*}\right)^{(0)}$. Then we have

$$
\begin{aligned}
\phi^{-1} \breve{w}_{\bar{i}} \phi(\lambda) & =\phi^{-1}\left(\phi(\lambda)-\phi(\lambda)\left(\widehat{h}_{\bar{i}}\right) \widehat{\alpha}_{\bar{i}}\right) \\
& =\phi^{-1}\left(\phi(\lambda)-\lambda\left(h_{i}\right) \widehat{\alpha}_{\bar{i}}\right) \\
& =\lambda-\lambda\left(h_{i}\right) \phi^{-1}\left(\widehat{\alpha}_{\bar{i}}\right) \\
& =\lambda-\lambda\left(h_{i}\right) \epsilon_{i} \sum_{k=0}^{N_{i}-1} \alpha_{\sigma^{k}(i)} \\
& =\bar{w}_{\bar{i}}(\lambda)
\end{aligned}
$$

which proves our assertion.

Proposition 8.4 The Weyl group $\breve{W}$ of the orbit Lie superalgebra $\breve{\mathfrak{g}}$ is isomorphic to the subgroup $\bar{W}$ of $W$ under the map $\breve{w}_{\bar{i}} \mapsto \bar{w}_{\bar{i}}\left(\bar{i} \in \breve{I}^{r e}\right)$.

Proof. See [13] or [14].

Remark. The original proof of 8.4 in [13] consists of a somewhat lengthy calculations. In [14, the proof was simplified and it was also proved that $\bar{W} \cong\{w \in$ $\left.W \mid w \sigma^{*}=\sigma^{*} w\right\}$.

Under the above identification of $\bar{W}$ with $\breve{W}$, we define the sign function $\bar{\varepsilon}: \bar{W} \rightarrow$ $\{ \pm 1\}$ of $\bar{W}$ by

$$
\bar{\varepsilon}(\bar{w})=\varepsilon\left(\phi \bar{w} \phi^{-1}\right)=\varepsilon\left(\psi^{-1}(\bar{w})\right) \text { for } \bar{w} \in \bar{W},
$$

where $\varepsilon$ denotes the sign function of the Weyl group $\breve{W}$ of the orbit Lie superalgebra $\breve{\mathfrak{g}}=\mathfrak{g}(\sigma)$. Note that, even though $\bar{W}$ is a subgroup of $W$, we use the sign function of $\breve{W}$, not the sign function of $W$. 


\section{Generalized Characters of Highest Weight Mod- ules for Dynkin Diagram Automorphisms}

Let $\Lambda \in P^{+}$be a dominant integral weight and let $M(\Lambda)$ be the Verma module with highest weight $\Lambda$. By construction of $M(\Lambda)$, we have

$$
M(\Lambda) \cong U(\mathfrak{g}) / R(\Lambda)
$$

where $R(\Lambda)$ is the left ideal of $U(\mathfrak{g})$ generated by the elements $e_{i k}\left(i \in I, k=1, \cdots, m_{i}\right)$ and $h-\Lambda(h) 1(h \in \mathfrak{h})$. By (8.1) and (8.2), we see that $\sigma(R(\Lambda))=R\left(\sigma^{*}(\Lambda)\right)$. Hence the Dynkin diagram automorphism $\sigma$ induces a $\mathbb{C}$-linear isomorphism

$$
\sigma: M(\Lambda) \rightarrow M\left(\sigma^{*}(\Lambda)\right)
$$

between Verma modules. Note that we have $\sigma\left(M(\Lambda)_{\lambda}\right)=M\left(\sigma^{*}(\Lambda)\right)_{\sigma^{*}(\lambda)}$ for all $\lambda \leq \Lambda$.

Similarly, let $V(\Lambda)$ be the irreducible highest weight module with highest weight $\Lambda$. Then by Corollary 6.4, the Dynkin diagram automorphism induces a $\mathbb{C}$-linear isomorphism

$$
\sigma: V(\Lambda) \rightarrow V\left(\sigma^{*}(\Lambda)\right)
$$

between irreducible highest weight modules, and we have $\sigma\left(V(\Lambda)_{\lambda}\right)=V\left(\sigma^{*}(\Lambda)\right)_{\sigma^{*}(\lambda)}$ for all $\lambda \leq \Lambda$.

In particular, if $\Lambda \in\left(\mathfrak{h}^{*}\right)^{(0)}$, i.e., if $\sigma^{*}(\Lambda)=\Lambda$, then the Dynkin diagram automorphism $\sigma$ induces $\mathbb{C}$-linear automorphisms on $M(\Lambda)$ and $V(\Lambda)$, and we can consider the generalized characters for $\sigma$ :

$$
\begin{aligned}
& \operatorname{ch}_{\sigma} M(\Lambda)=\sum_{\lambda \leq \Lambda} \operatorname{tr}\left(\sigma \mid M(\Lambda)_{\lambda}\right) e^{\lambda} \\
& \operatorname{ch}_{\sigma} V(\Lambda)=\sum_{\lambda \leq \Lambda} \operatorname{tr}\left(\sigma \mid V(\Lambda)_{\lambda}\right) e^{\lambda} .
\end{aligned}
$$

Note that we have only to take the sum over the weights $\lambda$ such that $\sigma^{*}(\lambda)=\lambda$.

Recall that the automorphism $\sigma^{*}$ permutes the roots of $\mathfrak{g}$ in such a way that we have

$$
\sigma^{*}\left(\Phi^{ \pm}\right)=\Phi^{ \pm}, \quad \sigma^{*}\left(\Phi_{0}^{ \pm}\right)=\Phi_{0}^{ \pm}, \quad \sigma^{*}\left(\Phi_{1}^{ \pm}\right)=\Phi_{1}^{ \pm} .
$$

Thus $\Phi^{ \pm}$is a disjoint union of $\sigma^{*}$-orbits of the elements in $\Phi^{ \pm}$. Let $S^{ \pm}$denote the set of representatives of $\sigma^{*}$-orbits in $\Phi^{ \pm}$and set

$$
S_{0}^{ \pm}=S^{ \pm} \cap \Phi_{0}, \quad S_{1}^{ \pm}=S^{ \pm} \cap \Phi_{1} .
$$

For each root $\alpha \in \Phi$, let $N_{\alpha}$ denote the number of elements in $\sigma^{*}$-orbit of $\alpha$, and define

$$
(\alpha)=\sum_{k=0}^{N_{\alpha}-1} \sigma^{* k}(\alpha) \text { and } \mathfrak{g}_{(\alpha)}=\bigoplus_{k=0}^{N_{\alpha}-1} \mathfrak{g}_{\sigma^{* k}(\alpha)}
$$


Then, by the Poincaré-Birkhoff-Witt Theorem, we have

$$
U\left(\mathfrak{g}^{ \pm}\right) \cong S\left(\mathfrak{g}_{0}^{ \pm}\right) \otimes \Lambda\left(\mathfrak{g}_{1}^{ \pm}\right)
$$

where

$$
S\left(\mathfrak{g}_{0}^{ \pm}\right) \cong \bigotimes_{\alpha \in S_{0}^{ \pm}} S\left(\mathfrak{g}_{(\alpha)}\right), \text { and } \Lambda\left(\mathfrak{g}_{1}^{ \pm}\right) \cong \bigotimes_{\beta \in S_{1}^{ \pm}} \Lambda\left(\mathfrak{g}_{(\beta)}\right)
$$

as $\mathbb{C}$-vector spaces. Hence the generalized (super)character of the Verma module $M(0)$ for the Dynkin diagram automorphism $\sigma$ is given by

$$
\begin{aligned}
\operatorname{ch}_{\sigma} M(0)= & \operatorname{sch}_{\sigma} M(0)=\sum_{\lambda \leq 0} \operatorname{tr}\left(\sigma \mid M(0)_{\lambda}\right) e^{\lambda} \\
= & \prod_{\alpha \in S_{0}^{+}}\left(\sum_{m \geq 0} \operatorname{tr}\left(\sigma \mid S^{m}\left(\mathfrak{g}_{(-\alpha)}\right)\right) e^{-m(\alpha) / N_{\alpha}}\right) \\
& \times \prod_{\beta \in S_{1}^{+}}\left(\sum_{n \geq 0} \operatorname{tr}\left(\sigma \mid \Lambda^{n}\left(\mathfrak{g}_{(-\beta)}\right)\right) e^{-n(\beta) / N_{\beta}}\right) \\
= & \prod_{\alpha \in S_{0}^{+}} \exp \left(\sum_{m \geq 1} \frac{1}{m} \operatorname{tr}\left(\sigma^{m} \mid \mathfrak{g}_{(-\alpha)}\right) e^{-m(\alpha) / N_{\alpha}}\right) \\
& \times \prod_{\beta \in S_{1}^{+}} \exp \left(-\sum_{n \geq 1} \frac{(-1)^{n}}{n} \operatorname{tr}\left(\sigma^{n} \mid \mathfrak{g}_{(-\beta)}\right) e^{-n(\beta) / N_{\beta}}\right) \\
= & \prod_{\alpha \in S^{+}} \exp \left(\sum_{m \geq 1} \frac{1}{m} \operatorname{str}\left(\sigma^{m} \mid \mathfrak{g}_{(-\alpha)}\right) E^{-m(\alpha) / N_{\alpha}}\right) \\
= & \prod_{\alpha \in \Phi^{+}} \exp \left(\sum_{m \geq 1} \frac{1}{m N_{\alpha}} \operatorname{str}\left(\sigma^{m} \mid \mathfrak{g}_{(-\alpha)}\right) E^{-m(\alpha) / N_{\alpha}}\right) .
\end{aligned}
$$

Note that if $\rho$ is a Weyl vector of the generalized Kac-Moody superalgebra $\mathfrak{g}$, then $\phi(\rho)$ is a Weyl vector of the orbit Lie superalgebra $\breve{\mathfrak{g}}$.

Proposition 9.1 For all $\bar{w} \in \bar{W}$, we have

$$
\bar{w}\left(e^{-\rho} c h_{\sigma} M(0)\right)=\bar{\varepsilon}(\bar{w}) e^{-\rho} c h_{\sigma} M(0) .
$$

Proof. It suffices to prove our assertion for the simple reflections $\bar{w}_{\bar{i}} \in \bar{W}\left(\bar{i} \in \breve{I}^{r e}\right)$. For each $i \in I^{r e}$, let $r_{i}=\exp \left(e_{i}^{\prime}\right) \exp \left(-f_{i}^{\prime}\right) \exp \left(e_{i}^{\prime}\right)$, where the elements $e_{i}^{\prime}, f_{i}^{\prime}$ and $h_{i}^{\prime}$ are defined by

$$
\begin{cases}e_{i}^{\prime}=e_{i}, \quad f_{i}^{\prime}=f_{i}, \quad h_{i}^{\prime}=h_{i} & \\ e_{i}^{\prime}=\frac{1}{4}\left[e_{i}, e_{i}\right], \quad f_{i}^{\prime}=\frac{1}{4}\left[f_{i}, f_{i}\right], \quad h_{i}^{\prime}=\frac{1}{2} h_{i} & \text { if } i \in I^{\text {even }}\end{cases}
$$


Then for each $i \in I$, the elements $e_{i}^{\prime}, f_{i}^{\prime}$, and $h_{i}^{\prime}$ generate the subalgebra of $\mathfrak{g}$ isomorphic to $\operatorname{sl}(2, \mathbb{C})$, and by the same argument in [30], we can verify that $r_{i} \in \operatorname{Aut}(\mathfrak{g})$ and $r_{i}\left(\mathfrak{g}_{\alpha}\right)=\mathfrak{g}_{w_{i}(\alpha)}$.

We first consider the case when $\epsilon_{i}=1$. (Recall that, in this case, $a_{i, \sigma(i)}=0$.) Suppose $\alpha \neq \alpha_{i}$ and $\alpha \neq 2 \alpha_{i}$. If we put $\bar{r}_{\bar{i}}=\prod_{k=0}^{N_{i}-1} r_{\sigma^{k}(i)}$, then since $a_{i, \sigma^{k}(i)}=0$, we get $r_{\sigma^{k^{\prime}}(i)} r_{\sigma^{k}(i)}=r_{\sigma^{k}(i)} r_{\sigma^{k^{\prime}}(i)}$. It follows that

$$
\operatorname{str}\left(\sigma^{m} \mid \mathfrak{g}_{(-\alpha)}\right)=\operatorname{str}\left(\bar{r}_{\bar{i}} \sigma^{m} \bar{r}_{\bar{i}}^{-1} \mid \mathfrak{g}_{\left(-\bar{w}_{\bar{i}} \alpha\right)}\right)=\operatorname{str}\left(\sigma^{m} \mid \mathfrak{g}_{\left(-\bar{w}_{\bar{i}} \alpha\right)}\right) .
$$

If $\alpha=\alpha_{i}$ and $i \in I^{e v e n}$, then, since

$$
\operatorname{str}\left(\sigma^{m} \mid \mathfrak{g}_{\left(-\alpha_{i}\right)}\right)= \begin{cases}N_{i} & \text { if } m \equiv 0\left(\bmod N_{i}\right) \\ 0 & \text { otherwise }\end{cases}
$$

we have

$$
\exp \left(\sum_{m=1}^{\infty} \frac{1}{m} \operatorname{str}\left(\sigma^{m} \mid \mathfrak{g}_{\left(-\alpha_{i}\right)}\right) E^{-m\left(\alpha_{i}\right) / N_{i}}\right)=\left(1-E^{-\left(\alpha_{i}\right)}\right)^{-1},
$$

and

$$
\bar{w}_{\bar{i}}\left(e^{-\rho}\left(1-E^{-\left(\alpha_{i}\right)}\right)^{-1}\right)=e^{-\rho+\left(\alpha_{i}\right)}\left(1-E^{\left(\alpha_{i}\right)}\right)^{-1}=-e^{-\rho}\left(1-E^{-\left(\alpha_{i}\right)}\right)^{-1} .
$$

Similarly, if $\alpha=\alpha_{i}$ or $\alpha=2 \alpha_{i}$ for $i \in I^{\text {odd }}$, then

$$
\begin{aligned}
& \exp \left(\sum_{m=1}^{\infty} \frac{1}{m} \operatorname{str}\left(\sigma^{m} \mid \mathfrak{g}_{\left(-\alpha_{i}\right)}\right) E^{-m\left(\alpha_{i}\right) / N_{i}}\right) \\
& \times \exp \left(\sum_{m=1}^{\infty} \frac{1}{m} \operatorname{str}\left(\sigma^{m} \mid \mathfrak{g}_{\left(-2 \alpha_{i}\right)}\right) E^{-m\left(2 \alpha_{i}\right) / N_{i}}\right) \\
& =\left(1-E^{-\left(\alpha_{i}\right)}\right)\left(1-E^{-\left(2 \alpha_{i}\right)}\right)^{-1},
\end{aligned}
$$

and

$$
\begin{aligned}
& \bar{w}_{\bar{i}}\left(e^{-\rho}\left(1-E^{-\left(\alpha_{i}\right)}\right)\left(1-E^{-\left(2 \alpha_{i}\right)}\right)^{-1}\right) \\
& \quad=e^{-\rho+\left(\alpha_{i}\right)}\left(1-E^{\left(\alpha_{i}\right)}\right)\left(1-E^{\left(2 \alpha_{i}\right)}\right)^{-1} \\
& \quad=-e^{-\rho+\left(\alpha_{i}\right)}\left(1+e^{\left(\alpha_{i}\right)}\right)\left(1-e^{\left(2 \alpha_{i}\right)}\right)^{-1} \\
& \quad=-e^{-\rho}\left(1-E^{-\left(\alpha_{i}\right)}\right)\left(1-E^{-\left(2 \alpha_{i}\right)}\right)^{-1} .
\end{aligned}
$$

Next, we consider the case when $\epsilon_{i}=2$. In this case, we have $a_{i, \sigma^{N_{i} / 2}(i)}=-1$, which implies that $i \in I^{\text {even }}$. Suppose $\alpha \neq \alpha_{k}$ and $\alpha \neq \alpha_{k}+\alpha_{\sigma^{N_{i} / 2}(k)}$ for any $k=\sigma^{l}(i)$ $\left(1 \leq l \leq N_{i} / 2\right)$. Set $\bar{r}_{\bar{i}}=\prod_{l=0}^{N_{i} / 2-1} r_{\sigma^{l}(i)} r_{\sigma^{l+N_{i} / 2}(i)} r_{\sigma^{l}(i)}$. Since $a_{k, \sigma^{N_{i} / 2}(k)}=-1$ implies $r_{k} r_{\sigma^{N_{i} / 2}(k)} r_{k}=r_{\sigma^{N_{i} / 2}(k)} r_{k} r_{\sigma^{N_{i} / 2}(k)}$ (see, for example, [33]), we have $\sigma \bar{r}_{\bar{i}}=\bar{r}_{\bar{i}} \sigma$ and

$$
\operatorname{str}\left(\sigma^{m} \mid \mathfrak{g}_{(-\alpha)}\right)=\operatorname{str}\left(\bar{r}_{\bar{i}} \sigma^{m} \bar{r}_{\bar{i}}^{-1} \mid \mathfrak{g}_{\left(-\bar{w}_{\bar{i}} \alpha\right)}\right)=\operatorname{str}\left(\sigma^{m} \mid \mathfrak{g}_{\left(-\bar{w}_{\bar{i}} \alpha\right)}\right) .
$$


If $\alpha=\alpha_{k}$ or $\alpha=\alpha_{k}+\alpha_{\sigma^{N_{i} / 2}(k)}$ for some $k=\sigma^{l}(i)\left(1 \leq l \leq N_{i} / 2\right)$ (i.e. $\left.(\alpha)=\left(\alpha_{i}\right)\right)$, then

$$
\begin{aligned}
& \exp \left(\sum_{m=1}^{\infty} \frac{1}{m} \operatorname{str}\left(\sigma^{m} \mid \mathfrak{g}_{\left(-\alpha_{i}\right)}\right) E^{-m\left(\alpha_{i}\right) / N_{i}}\right)=\left(1-E^{-\left(\alpha_{i}\right)}\right)^{-1} \\
& \exp \left(\sum_{m=1}^{\infty} \frac{1}{m} \operatorname{str}\left(\sigma^{m} \mid \mathfrak{g}_{\left(-\alpha_{i}-\alpha_{\sigma^{N_{i} / 2}(i)}\right)}\right) E^{-2 m\left(\alpha_{i}+\alpha_{\sigma^{N_{i} / 2}(i)}\right) / N_{i}}\right)=\left(1+E^{-\left(\alpha_{i}\right)}\right)^{-1},
\end{aligned}
$$

and

$$
\begin{aligned}
& \bar{w}_{\bar{i}}\left(e^{-\rho}\left(1-E^{\left(-\alpha_{i}\right)}\right)^{-1}\left(1+E^{\left(-\alpha_{i}\right)}\right)^{-1}\right) \\
& \quad=-e^{-\rho}\left(1-E^{\left(-\alpha_{i}\right)}\right)^{-1}\left(1+E^{\left(-\alpha_{i}\right)}\right)^{-1},
\end{aligned}
$$

which completes the proof of our assertion.

Proposition 9.2 For $\Lambda \in P^{+}$and $\bar{w} \in \bar{W} \subset W$, we have

$$
\bar{w}\left(\operatorname{ch}_{\sigma} V(\Lambda)\right)=\operatorname{ch}_{\sigma} V(\Lambda) .
$$

Proof. It suffices to prove (9.6) for $\bar{w}=\bar{w}_{\bar{i}}\left(\bar{i} \in \breve{I}^{r e}\right)$. Let $\pi: \mathfrak{g} \rightarrow g l(V(\Lambda))$ denote the representation of $\mathfrak{g}$ on $V(\Lambda)$ and define

$$
\bar{r}_{i}^{\pi}= \begin{cases}\prod_{k=0}^{N_{i}-1} r_{\sigma^{k}(i)}^{\pi} & \text { if } \epsilon_{i}=1 \\ \prod_{l=0}^{N_{i} / 2-1} r_{\sigma^{l}(i)} r_{\sigma^{l+N_{i} / 2}(i)} r_{\sigma^{l}(i)} & \text { if } \epsilon_{i}=2\end{cases}
$$

By the same argument in the preceding proposition, we have $\sigma \bar{r}_{\bar{i}}^{\pi}=\bar{r}_{\bar{i}}^{\pi} \sigma$. It follows that

$$
\operatorname{tr}\left(\sigma \mid V(\Lambda)_{\lambda}\right)=\operatorname{tr}\left(\sigma \mid V(\Lambda)_{\bar{w}_{\bar{i}} \lambda}\right)
$$

which proves our assertion.

Now, we are in a position to state and prove the main result of this section.

Theorem 9.3 Let $\sigma$ be a Dynkin diagram automorphism of a generalized Kac-Moody superalgebra $\mathfrak{g}$ and let $\breve{\mathfrak{g}}=\mathfrak{g}(\sigma)$ be the corresponding orbit Lie superalgebra. Let $\phi$ : $\left(\mathfrak{h}^{*}\right)^{(0)} \rightarrow \widehat{\mathfrak{h}}^{*}$ be the $\mathbb{C}$-linear isomorphism given by $(8.6)$, and extend it to a $\mathbb{C}$-linear map $\phi: \mathbb{C}\left[\left[\left(\mathfrak{h}^{*}\right)^{(0)}\right]\right] \rightarrow \mathbb{C}\left[\left[\widehat{\mathfrak{h}}^{*}\right]\right]$ by $\phi\left(e^{\lambda}\right)=e^{\phi(\lambda)}$ for $\lambda \in\left(\mathfrak{h}^{*}\right)^{(0)}$. Then, for a symmetric dominant integral weight $\Lambda \in P^{+} \cap\left(\mathfrak{h}^{*}\right)^{(0)}$, we have

$$
\phi\left(\operatorname{ch}_{\sigma} V(\Lambda)\right)=\operatorname{ch} \breve{V}(\phi(\Lambda))
$$

where $\breve{V}(\phi(\Lambda))$ is the irreducible highest weight $\breve{\mathfrak{g}}$-module with highest weight $\phi(\Lambda)$. 
Proof. Using the properties of Casimir operator given in Proposition 6.2, the same argument in 31 yields

$$
\operatorname{ch}_{\sigma} V(\Lambda)=\sum_{\substack{\lambda \leq \Lambda \\|\lambda+\rho|^{2}=|\Lambda+\rho|^{2}}} c_{\lambda} \operatorname{ch}_{\sigma} M(\lambda)
$$

Since $\operatorname{ch}_{\sigma} M(\lambda)=e^{\lambda} \operatorname{ch}_{\sigma} M(0)$, by (9.8), we have

$$
e^{\rho} R_{\sigma} \operatorname{ch}_{\sigma} V(\Lambda)=\sum_{\substack{\lambda \leq \Lambda \\|\lambda+\rho|^{2}=|\Lambda+\rho|^{2}}} c_{\lambda} e^{\lambda+\rho}
$$

where

$$
R_{\sigma}=\left(\operatorname{ch}_{\sigma} M(0)\right)^{-1}=\prod_{\alpha \in \Phi^{+}} \exp \left(-\sum_{m \geq 1} \frac{1}{m N_{\alpha}} \operatorname{str}\left(\sigma^{m} \mid \mathfrak{g}_{(-\alpha)}\right) E^{-m(\alpha) / N_{\alpha}}\right) .
$$

We may assume that the sum in the right-hand side of (9.9) is taken over the weights $\lambda$ which are invariant under $\sigma^{*}$.

If $\bar{w}(\lambda+\rho)=\tau+\rho$, then $c_{\tau}=\bar{\varepsilon}(\bar{w}) c_{\lambda}$. Choose a weight $\tau$ such that $c_{\tau} \neq 0$. Then $\bar{w}(\tau+\rho) \leq \Lambda+\rho$ for all $\bar{w} \in \bar{W}$. Let $\lambda$ be an element of the set $\{\bar{w}(\tau+\rho)-\rho \mid \bar{w} \in \bar{W}\}$ such that $\operatorname{ht}(\Lambda-\lambda)$ is minimal. Note that $\left(\lambda+\rho \mid \alpha_{i}\right) \geq 0$ for all $\bar{i} \in \widehat{I}^{r e} \backslash \breve{I}^{\text {re }}$ by definition of $\breve{I}$. If $\left(\lambda+\rho \mid \alpha_{i}\right)<0$ for some $\bar{i} \in \breve{I}^{r e}$, then $\bar{w}_{\bar{i}}(\lambda+\rho)=\lambda+\rho-\epsilon_{i}(\lambda+\rho)\left(h_{i}\right)\left(\alpha_{i}\right)$ and $\operatorname{ht}\left(\Lambda-\bar{w}_{\bar{i}}(\lambda)\right)<\operatorname{ht}(\Lambda-\lambda)$, which is a contradiction. Hence, $\left(\lambda+\rho \mid \alpha_{i}\right) \geq 0$ for all $i \in I^{r e}$. Moreover, since $|\Lambda+\rho|^{2}=|\lambda+\rho|^{2}$, we have

$$
0=|\Lambda+\rho|^{2}-|\lambda+\rho|^{2}=(\Lambda \mid \Lambda-\lambda)+(\lambda+2 \rho \mid \Lambda-\lambda),
$$

where $\Lambda-\lambda=\sum_{i \in I} n_{i} \alpha_{i}$ lies in $Q^{+}$.

If $i \in I^{r e}$, then

$$
\left(\lambda+2 \rho \mid \alpha_{i}\right)=\left(\lambda+\rho \mid \alpha_{i}\right)+\left(\rho \mid \alpha_{i}\right)>0 .
$$

If $i \in I^{i m}$ and $n_{i} \geq 1$, then

$$
\begin{aligned}
& \left(\lambda+2 \rho \mid \alpha_{i}\right)=\left(\lambda+\alpha_{i} \mid \alpha_{i}\right) \\
& =\left(\Lambda-\sum_{j \in I \backslash\{i\}} n_{j} \alpha_{j}-\left(n_{i}-1\right) \alpha_{i} \mid \alpha_{i}\right) \\
& =\left(\Lambda \mid \alpha_{i}\right)-\sum_{j \in I \backslash\{i\}} n_{j}\left(\alpha_{j} \mid \alpha_{i}\right)-\left(n_{i}-1\right)\left(\alpha_{i} \mid \alpha_{i}\right) \geq 0 .
\end{aligned}
$$

It follows that $n_{i}=0$ for all $i \in I^{r e}$. That is, $\lambda=\Lambda-\sum_{i \in I^{i m}} n_{i} \alpha_{i}$. ¿From (9.10), for $k \in I^{i m}$ with $n_{k} \geq 1$, we have

$$
\left(\lambda+2 \rho \mid \alpha_{k}\right)=\left(\lambda+\alpha_{k} \mid \alpha_{k}\right)=\left(\Lambda \mid \alpha_{k}\right)-\sum_{i \in I^{i m}} n_{i}^{\prime}\left(\alpha_{i} \mid \alpha_{k}\right)=0,
$$


where $n_{i}^{\prime}=n_{i}$ for $i \neq k$ and $n_{k}^{\prime}=n_{k}-1$. Hence we have

$$
\begin{aligned}
\left(\Lambda \mid \alpha_{k}\right) & =0 \\
\left(\alpha_{i} \mid \alpha_{k}\right) & =0 \text { if } n_{i} \geq 1 \text { and } i \neq k \\
\left(\alpha_{k} \mid \alpha_{k}\right) & =0 \text { if } n_{k} \geq 2
\end{aligned}
$$

and $k$ lies in $\breve{I}^{i m}$. Combining the above conditions and the fact that $n_{i}=n_{\sigma(i)}$ for all $i \in I$,we conclude that $\lambda$ has the form

$$
\lambda=\Lambda-\sum_{\bar{i} \in \breve{I}^{i m}} n_{\bar{i}}\left(\alpha_{i}\right)
$$

where

$$
\begin{aligned}
\left(\left(\alpha_{i}\right) \mid\left(\alpha_{j}\right)\right) & =0 \text { if } \bar{i} \neq \bar{j} \text { and } n_{\bar{i}}, n_{\bar{j}} \geq 1 \\
\left(\Lambda \mid\left(\alpha_{i}\right)\right) & =0 \text { if } n_{\bar{i}} \geq 1 \\
\left(\left(\alpha_{i}\right) \mid\left(\alpha_{i}\right)\right) & =0 \text { if } n_{\bar{i}} \geq 2 .
\end{aligned}
$$

Let $\lambda, \lambda^{\prime}$ be two such elements of the above form. Suppose that there exists a $\bar{w} \in \bar{W}$ such that $\bar{w}(\lambda+\rho)=\lambda^{\prime}+\rho$. Then, if $\lambda \in P^{+}$, we have $\bar{w}(\lambda) \leq \lambda$, since $\bar{w}(\lambda)$ is also a weight of the $\mathfrak{g}$-module $V(\lambda)$. Furthermore, by induction on the length of $\bar{w}$, we can verify $\bar{w}(\rho) \leq \rho$. Hence we obtain $\bar{w}(\lambda+\rho) \leq \lambda+\rho$ and $\bar{w}(\lambda+\rho)=\lambda+\rho-\sum_{\alpha \in \Phi^{+, r e}} n_{\alpha} \alpha$ with $n_{\alpha} \geq 0$. On the other hand, if $\bar{w}(\lambda+\rho)=\lambda^{\prime}+\rho$, we must have $n_{\alpha}=0$ for all $\alpha \in \Phi^{+, r e}$. Hence $\bar{w}(\lambda+\rho)=\lambda+\rho$, which implies $\lambda=\lambda^{\prime}$.

Let $\Phi^{+}(\Lambda)$ be the set defined in (6.12) and let $\Phi^{+}(\Lambda)^{(0)}=\Phi^{+}(\Lambda) \cap\left(\mathfrak{h}^{*}\right)^{(0)}$ be the set of elements $\sum_{\bar{i} \in \breve{I}^{i m}} n_{\bar{i}}\left(\alpha_{i}\right)$ such that $\left(\left(\alpha_{i}\right) \mid\left(\alpha_{j}\right)\right)=0$ if $n_{\bar{i}}, n_{\bar{j}} \geq 1$ for $\bar{i} \neq \bar{j},\left(\Lambda \mid\left(\alpha_{i}\right)\right)=0$ if $n_{\bar{i}} \geq 1$, and $\left(\left(\alpha_{i}\right) \mid\left(\alpha_{i}\right)\right)=0$ if $n_{\bar{i}} \geq 2$. So (9.9) can be written as follows:

$$
e^{\rho} R_{\sigma} \operatorname{ch}_{\sigma} V(\Lambda)=\sum_{\bar{w} \in \bar{W}} \bar{\epsilon}(\bar{w}) \sum_{\beta \in \Phi^{+}(\Lambda)^{(0)}} c_{\Lambda-\beta} e^{\bar{w}(\Lambda-\beta+\rho)} .
$$

Now, it remains to determine $c_{\Lambda-\beta}$ for $\beta \in S_{\Lambda}^{(0)}$. First, we observe that $\operatorname{ch}_{\sigma} V(\Lambda)$ contains no term of the form $e^{\Lambda-\sum_{i \in I^{i m}} n_{i} \alpha_{i}}$, where $\left(\Lambda \mid \alpha_{i}\right)=0$ for all $i$ such that $n_{i} \geq 1$. So, in order to determine $c_{\Lambda-\beta}$, we have only to compute the coefficient of $e^{\Lambda-\beta}$ in $R_{\sigma}$. Note that $\left(\left(\alpha_{i}\right) \mid\left(\alpha_{j}\right)\right)=0$ implies that $\alpha_{i}+\alpha_{j} \notin \Phi$.

For each $\bar{i} \in \breve{I}^{i m}$, we have

$$
\exp \left(-\sum_{m \geq 1} \frac{1}{m} \operatorname{str}\left(\sigma^{m} \mid \mathfrak{g}_{\left(-\alpha_{i}\right)}\right) E^{-m\left(\alpha_{i}\right) / N_{i}}\right)=\left(1-E^{\left(-\alpha_{i}\right)}\right)^{m_{i}}
$$

For $\beta=\sum_{\bar{i} \in \breve{I}^{i m}} n_{\bar{i}}\left(\alpha_{i}\right)$, using the formal power series expansions

$$
(1-x)^{n}=\sum_{k=0}^{n}\left(\begin{array}{l}
n \\
k
\end{array}\right)(-1)^{k} x^{k}
$$


and

$$
(1+x)^{-n}=\sum_{k=0}^{\infty}\left(\begin{array}{c}
n+k-1 \\
k
\end{array}\right)(-1)^{k} x^{k}
$$

we obtain

$$
c_{\Lambda-\beta}=\prod_{\bar{i} \in \breve{I}^{\text {even }}}\left(\begin{array}{c}
m_{\bar{i}} \\
n_{\bar{i}}
\end{array}\right)(-1)^{n_{\bar{i}}} \prod_{\bar{i} \in \breve{I}^{\text {odd }}}\left(\begin{array}{c}
m_{\bar{i}}+n_{\bar{i}}-1 \\
n_{\bar{i}}
\end{array}\right)(-1)^{n_{\bar{i}}}=(-1)^{|\phi(\beta)|} \varepsilon(\phi(\beta)) .
$$

When $\Lambda=0$, we get

$$
R_{\sigma}=\sum_{\bar{w} \in \bar{W}} \bar{\varepsilon}(\bar{w}) \sum_{\lambda \in \Phi^{+}(0)^{(0)}} c_{-\lambda} e^{\bar{w}(\lambda+\rho)-\rho} .
$$

Since $\phi\left(\left(\alpha_{i}\right)\right)=\widehat{\alpha}_{\bar{i}}$ for $\bar{i} \in \breve{I}^{i m}$, by Lemma , we have $\left(\left(\alpha_{i}\right) \mid\left(\alpha_{j}\right)\right)=0$ if and only if $\left(\widehat{\alpha}_{\bar{i}} \mid \widehat{\alpha}_{\bar{j}}\right)=0$ and $\left(\Lambda \mid\left(\alpha_{i}\right)\right)=0$ if and only if $\left(\phi(\Lambda) \mid \widehat{\alpha}_{\bar{i}}\right)=0$. It follows that $\phi\left(\Phi^{+}(\Lambda)^{(0)}\right)=$ $\breve{\Phi}^{+}(\phi(\Lambda)) \subset \widehat{\mathfrak{h}}^{*}$. Therefore we obtain

$$
\begin{aligned}
\phi\left(\operatorname{ch}_{\sigma} V(\Lambda)\right) & =\frac{\sum_{\bar{w} \in \bar{W}} \bar{\varepsilon}(\bar{w}) \sum_{\beta \in \Phi^{+}(\Lambda)(0)} c_{\Lambda-\beta} e^{\phi(\bar{w}(\Lambda-\beta+\rho)-\rho)}}{\sum_{\bar{w} \in \bar{W}} \bar{\varepsilon}(\bar{w}) \sum_{\gamma \in \Phi^{+}(0)^{(0)}} c_{-\gamma} e^{\phi(\bar{w}(-\gamma+\rho)-\rho)}} \\
& =\frac{\sum_{\breve{w} \in \widehat{W}} \varepsilon(\widehat{w}) \sum_{\widehat{\beta} \in \breve{\Phi}^{+}(\phi(\Lambda))} c_{\Lambda-\phi^{-1}(\widehat{\beta})} e^{\breve{w}(\phi(\Lambda)-\widehat{\beta}+\rho)-\rho}}{\sum_{\breve{w} \in \breve{W}} \varepsilon(\breve{w}) \sum_{\widehat{\gamma} \in \breve{\Phi}^{+}(0)} c_{-\phi^{-1}(\widehat{\gamma})} e^{\breve{w}(-\widehat{\gamma}+\rho)-\rho}} \\
& =\frac{\sum_{\breve{w} \in \breve{W}} \varepsilon(\breve{w}) \sum_{\widehat{\beta} \in \breve{\Phi}^{+}(\phi(\Lambda))}(-1)^{|\widehat{\beta}|} \varepsilon(\widehat{\beta}) e^{\breve{w}(\phi(\Lambda)-\widehat{\beta}+\rho)-\rho}}{\sum_{\breve{w} \in \breve{W}} \varepsilon(\breve{w}) \sum_{\widehat{\gamma} \in \breve{\Phi}^{+}(0)}(-1)^{|\widehat{\gamma}|} \varepsilon(\widehat{\gamma}) e^{\breve{w}(-\widehat{\gamma}+\rho)-\rho}} \\
& =\operatorname{ch} \breve{V}(\phi(\Lambda)) .
\end{aligned}
$$

\section{Corollary 9.4}

$$
\begin{aligned}
& \phi\left(R_{\sigma}\right)=\prod_{\alpha \in \breve{\Phi}^{+}}\left(1-E^{-\alpha}\right)^{\operatorname{sim} \breve{\mathfrak{g}}_{\alpha},} \\
& \phi\left(\operatorname{ch}_{\sigma} M(\Lambda)\right)=\operatorname{ch} \breve{M}(\phi(\Lambda)) .
\end{aligned}
$$

Proof. In the proof of Theorem 9.3, we have shown that

$$
R_{\sigma}=\sum_{\bar{w} \in \bar{W}} \bar{\varepsilon}(\bar{w}) \sum_{\gamma \in \Phi^{+}(0)^{(0)}} c_{-\gamma} e^{\bar{w}(-\gamma+\rho)-\rho} .
$$

Hence we have

$$
\begin{aligned}
\phi\left(R_{\sigma}\right) & =\sum_{\bar{w} \in \bar{W}} \bar{\varepsilon}(\bar{w}) \sum_{\gamma \in \Phi^{+}(0)^{(0)}} c_{-\gamma} e^{\phi(\bar{w}(-\gamma+\rho)-\rho)}=\sum_{\breve{w} \in \breve{W}} \varepsilon(\breve{w}) \sum_{\widehat{\gamma} \in \breve{\Phi}^{+}(0)} c_{-\phi^{-1}(\widehat{\gamma})} e^{\breve{w}(-\widehat{\gamma}+\rho)-\rho} \\
& =\sum_{\breve{w} \in \breve{W}} \varepsilon(\breve{w}) \sum_{\widehat{\gamma} \in \breve{\Phi}^{+}(0)}(-1)^{|\widehat{\gamma}|} \varepsilon(\widehat{\gamma}) e^{\breve{w}(-\widehat{\gamma}+\rho)-\rho}=\prod_{\alpha \in \breve{\Phi}^{+}}\left(1-E^{-\alpha}\right)^{\operatorname{sdim} \breve{g}_{\alpha}} .
\end{aligned}
$$


Since $\operatorname{ch}_{\sigma} M(\Lambda)=e^{\Lambda} R_{\sigma}^{-1}$, we obtain

$$
\phi\left(\operatorname{ch}_{\sigma} M(\Lambda)\right)=e^{\phi(\Lambda)} \prod_{\alpha \in \breve{\Phi}^{+}}\left(1-E^{-\alpha}\right)^{-\operatorname{sdim} \breve{g}_{\alpha}}=\operatorname{ch} \breve{M}(\phi(\Lambda)) .
$$

Set $\widehat{Q}=\bigoplus_{i \in \widehat{I}} \mathbb{Z}\left(\widehat{\alpha}_{\bar{i}} / \epsilon_{i} N_{i}\right)$, and define a $\mathbb{Z}$-linear map $\widehat{\phi}: Q \rightarrow \widehat{Q}$ by

$$
\widehat{\phi}\left(\alpha_{i}\right)=\widehat{\alpha}_{\bar{i}} / \epsilon_{i} N_{i} \text { for } i \in I, k=0,1, \cdots, N_{i}-1
$$

Then $\widehat{\phi}$ is an extension of $\left.\phi\right|_{Q \cap\left(\mathfrak{h}^{*}\right)^{(0)}}$ to $Q$. From now on, we will also use $\phi$ for $\widehat{\phi}$.

For each $\beta \in \widehat{Q}$, define the subspace $\mathfrak{g}_{[\alpha]}$ of $\mathfrak{g}$ to be

$$
\mathfrak{g}_{[\beta]}=\bigoplus_{\alpha \in \phi^{-1}(\beta)} \mathfrak{g}_{\alpha}
$$

Clearly, $\left[\mathfrak{g}_{[\alpha]}, \mathfrak{g}_{[\beta]}\right] \subset \mathfrak{g}_{[\alpha+\beta]}$, and hence $\mathfrak{g}$ becomes a $\widehat{Q}$-graded Lie superalgebra with the coloring of $\widehat{Q}$ induced by that of $Q$. Note that the Dynkin diagram automorphism $\sigma$ preserves the $\widehat{Q}$-gradation of $\mathfrak{g}$. With respect to this gradation, the generalized denominator identity for the Dynkin diagram automorphism $\sigma$ of the generalized KacMoody superalgebra $\mathfrak{g}$ is the same as

$$
\prod_{\beta \in \widehat{Q}^{+}} \exp \left(-\sum_{m=1}^{\infty} \frac{1}{m} \operatorname{str}\left(\sigma^{m} \mid \mathfrak{g}_{[-\beta]}\right) E^{-m \beta}\right)=1-\operatorname{sch}_{\sigma} H\left(\mathfrak{g}_{-}\right) .
$$

On the other hand, we have

$$
\begin{aligned}
\phi\left(R_{\sigma}\right) & =\prod_{\alpha \in \Phi^{+}} \exp \left(-\sum_{m=1}^{\infty} \frac{1}{m N_{\alpha}} \operatorname{str}\left(\sigma^{m} \mid \mathfrak{g}_{(-\alpha)}\right) E^{-m \phi((\alpha)) / N_{\alpha}}\right) \\
& =\prod_{\alpha \in \Phi^{+}} \exp \left(-\sum_{m=1}^{\infty} \frac{1}{m N_{\alpha}} \operatorname{str}\left(\sigma^{m} \mid \mathfrak{g}_{(-\alpha)}\right) E^{-m \phi(\alpha)}\right) \\
& =\prod_{\beta \in \widehat{Q}^{+}} \exp \left(-\sum_{m=1}^{\infty} \frac{1}{m} \operatorname{str}\left(\sigma^{m} \mid \mathfrak{g}_{[-\beta]}\right) E^{-m \beta}\right) .
\end{aligned}
$$

Therefore, by combining (9.12) and (9.13) with Corollary 9.4, we obtain:

Theorem 9.5 The generalized denominator identity of the $\widehat{Q}$-graded generalized KacMoody superalgebra $\mathfrak{g}$ for the Dynkin diagram automorphism $\sigma$ is the same as the denominator identity of the orbit Lie superalgebra $\breve{\mathfrak{g}}$.

Remark. (a) For the Kac-Moody algebras and generalized Kac-Moody algebras, Theorem 9.3 was proved in [13] and [14]. They also gave a list of Dynkin diagram 
automorphisms of Kac-Moody algebras $\mathfrak{g}$ of finite growth and corresponding orbit Lie algebras $\breve{\mathfrak{g}}([\mathbb{1 3}])$.

(b) Let $\mathfrak{D}_{\sigma}(\mathfrak{g})$ denote the generalized denominator identity of the $\widehat{Q}$-graded generalized Kac-Moody superalgebra $\mathfrak{g}$ for the Dynkin diagram automorphism $\sigma$ and $\mathfrak{D}(\breve{\mathfrak{g}})$ the denominator identity of the orbit Lie superalgebra $\breve{\mathfrak{g}}$. Then, Theorem 9.5 implies

$$
\mathfrak{D}_{\sigma}(\mathfrak{g})=\mathfrak{D}(\breve{\mathfrak{g}}) .
$$

(c) For the affine Kac-Moody superalgebras introduced in [30], we have

$$
\begin{aligned}
\mathfrak{D}_{\sigma}\left(A^{(2)}(0,2 n-1)\right) & =\mathfrak{D}\left(B^{(1)}(0, n-1)\right) \quad \text { when } n \geq 2 \text { and } \sigma \text { has order } 2, \\
\mathfrak{D}_{\sigma}\left(C^{(2)}(3)\right) & =\mathfrak{D}\left(A^{(4)}(0,2)\right) \quad \text { when } \sigma \text { has order } 2, \\
\mathfrak{D}_{\sigma}\left(C^{(2)}(0,2 n+1)\right) & =\mathfrak{D}\left(A^{(4)}(0,2 n)\right) \quad \text { when } n \geq 2 \text { and } \sigma \text { has order } 2, \\
\mathfrak{D}_{\sigma}\left(C^{(2)}(0,2 n+2)\right) & =\mathfrak{D}\left(B^{(1)}(0, n)\right) \quad \text { when } n \geq 2 \text { and } \sigma \text { has order } 2 .
\end{aligned}
$$

The identity (9.14) can be rewritten as

$$
\prod_{\beta \in \widehat{Q}^{+}} \exp \left(-\sum_{m=1}^{\infty} \frac{1}{m} \operatorname{str}\left(\sigma^{m} \mid \mathfrak{g}_{[-\beta]}\right) E^{-m \beta}\right)=\prod_{\alpha \in \widehat{Q}^{+}}\left(1-E^{-\alpha}\right)^{\operatorname{sdim} \mathfrak{g}(\sigma)_{\alpha}},
$$

where $\mathfrak{g}(\sigma)=\breve{\mathfrak{g}}$ is the orbit Lie superalgebra corresponding to the Dynkin diagram automorphism $\sigma$ of $\mathfrak{g}$. Let us consider $\mathfrak{g}\left(\sigma^{d}\right)$ for a nonnegative integer $d$. Then we have the following objects as in the case of $\mathfrak{g}(\sigma)$ :

(i) $\widehat{I}(d), \breve{I}(d) N_{i}(d), \epsilon_{i}(d), \widehat{A}(d), \breve{A}(d), \widehat{Q}(d)=\bigoplus_{\bar{i} \in \widehat{I}(d)} \mathbb{Z}\left(\widehat{\alpha}_{\bar{i}} / \epsilon_{i}(d) N_{i}(d)\right)$,

(ii) a $\mathbb{Z}$-linear map $\phi_{d}: Q \rightarrow \widehat{Q}(d)$, where $\phi_{d}\left(\alpha_{i}\right)=\widehat{\alpha}_{\bar{i}} / \epsilon_{i}(d) N_{i}(d)$ for $i \in I, k=$ $1, \cdots, N_{i}(d)$.

Applying the above equation to $\mathfrak{g}\left(\sigma^{d}\right)$, we get

$$
\prod_{\beta \in \widehat{Q}^{+}(d)} \exp \left(-\sum_{m=1}^{\infty} \frac{1}{m} \operatorname{str}\left(\sigma^{m d} \mid \mathfrak{g}_{[-\beta]_{d}}\right) E^{-m \beta}\right)=\prod_{\alpha \in \widehat{Q}^{+}(d)}\left(1-E^{-\alpha}\right)^{\operatorname{sdim} \mathfrak{g}\left(\sigma^{d}\right)_{\alpha}},
$$

where $\mathfrak{g}_{[\beta]_{d}}=\bigoplus_{\alpha \in \phi_{d}^{-1}(\beta)} \mathfrak{g}_{\alpha}$. Also, if we define a map $\tau$ of $\widehat{I}(d)$ by $\tau(\bar{i})=\overline{\sigma(i)}$, then it induces a $\mathbb{Z}$ - linear map $\phi^{d}: \widehat{Q}(d) \rightarrow \widehat{Q}$ such that $\phi^{d} \phi_{d}(\beta)=\phi(\beta)$ for all $\beta \in Q$. By taking $\phi^{d}$, (9.15) can be written as

$$
\prod_{\beta \in \widehat{Q}^{+}} \exp \left(-\sum_{m=1}^{\infty} \frac{1}{m} \operatorname{str}\left(\sigma^{m d} \mid \mathfrak{g}_{[-\beta]}\right) E^{-m \beta}\right)=\prod_{\alpha \in \widehat{Q}^{+}}\left(1-E^{-\alpha}\right)^{\operatorname{sdim} \mathfrak{g}\left(\sigma^{d}\right)_{[\alpha]}^{d}},
$$

where $\mathfrak{g}\left(\sigma^{d}\right)_{[\alpha]^{d}}=\bigoplus_{\beta \in\left(\phi^{d}\right)^{-1}(\alpha)} \mathfrak{g}\left(\sigma^{d}\right)_{\beta}$. By taking the logarithm on both sides, we have

$$
\sum_{\beta \in \widehat{Q}^{+}} \sum_{m=1}^{\infty} \frac{1}{m} \operatorname{str}\left(\sigma^{m d} \mid \mathfrak{g}_{[-\beta]}\right) E^{-m \beta}=\sum_{\alpha \in \widehat{Q}^{+}} \sum_{n=1}^{\infty} \frac{1}{n} \operatorname{sdim} \mathfrak{g}\left(\sigma^{d}\right)_{[\alpha]^{d}} E^{-n \alpha} .
$$


It follows that

$$
\sum_{\substack{k>0 \\ \beta=k \tau}} \frac{1}{k} \operatorname{str}\left(\sigma^{d k} \mid \mathfrak{g}_{[-\tau]}\right)=\sum_{\substack{a>0 \\ \beta=a \gamma}} \frac{1}{a} \operatorname{sdim} \mathfrak{g}\left(\sigma^{d}\right)_{[\gamma]^{d}} .
$$

If we put $T_{\sigma^{d}}(\beta)=\sum_{a \mid \beta} \frac{1}{a} \operatorname{sdim} \mathfrak{g}\left(\sigma^{d}\right)_{[\beta / a]^{d}}$ for a nonnegative integer $d$ and $\beta \in \widehat{Q}$, then by Möbius inversion

$$
\begin{aligned}
\operatorname{str}\left(\sigma^{k} \mid \mathfrak{g}_{[-\alpha]}\right) & =\sum_{d \mid \alpha} \frac{1}{d} \mu(d) T_{\sigma^{d k}}(\alpha / d) \\
& =\sum_{a d \mid \alpha} \frac{1}{a d} \mu(d) \operatorname{sdim} \mathfrak{g}\left(\sigma^{d k}\right)_{[\alpha / a d]},
\end{aligned}
$$

where $\mu$ denotes the classical Möbius function.

Let $\mathfrak{g}^{\sigma}$ be the fixed point subalgebra of $\mathfrak{g}$ for a Dynkin diagram automorphism $\sigma$. Then it is also graded by $\widehat{Q}$ and by (9.17) we get

Proposition 9.6 For each $\beta \in \widehat{Q}$,

$$
\operatorname{sdim}_{\mathfrak{g}_{\beta}}^{\sigma}=\frac{1}{|\sigma|} \sum_{k=1}^{|\sigma|} \sum_{a d \mid \beta} \frac{1}{a d} \mu(d) \operatorname{sdim} \mathfrak{g}\left(\sigma^{d k}\right)_{[\beta / a d]^{d k}},
$$

Proof. Note that $\mathfrak{g}_{\beta}^{\sigma}$ is a representation of a finite cyclic group $\langle\sigma\rangle$. Hence $\operatorname{dim} \mathfrak{g}_{\beta}^{\sigma}$ is the multiplicity of the trivial representation of $\langle\sigma\rangle$ in $\mathfrak{g}_{\beta}^{\sigma}$ and it is the same as the average of traces of $\tau \in\langle\sigma\rangle$ on $\mathfrak{g}_{\beta}^{\sigma}$.

Remark. By the similar argument in [5], we can see that $\mathfrak{g}^{\sigma}$ is a central extension of a generalized Kac-Moody superalgebra. However, the corresponding Borcherds-Cartan matrix is not known in general. Also, the decomposition of $\mathfrak{g}^{\sigma}$ with respect to the $\widehat{Q}$-gradation is coarser than its root space decomposition.

\section{References}

[1] D. Alexander, C. Cummins, J. McKay, C. Simons, Completely replicable functions, in Groups, Combinatorics, and Geometry, Durham Symposium, 1990, Lond. Math. Soc. Lecture Note Series 165 (1992), Cambridge Univ. Press, 87-98.

[2] Y. A. Bahturin, A. A. Mikhalev, V. M. Petrogradsky, M. V. Zaicev, Infinite dimensional Lie superalgebras, Walter de Gruyter, 1992.

[3] Y. A. Bahturin, Identical relations in Lie algebras, VNU Science Press, Utrecht, 1987. 
[4] R. E. Borcherds, Vertex algebras, Kac-Moody algebras and the monster, Proc. Natl. Acad. Sci. USA 83 (1986), 3068-3071.

[5] R. E. Borcherds, Generalized Kac-Moody algebras, J. Algebra 115 (1988), 501-512.

[6] R. E. Borcherds, Monstrous moonshine and monstrous Lie superalgebras, Invent. Math. 109 (1992), 405-44.

[7] R. E. Borcherds, Automorphic forms on $O_{s+2,2}(\mathbb{R})$ and infinite products, Invent. Math. 120 (1995), 161-213.

[8] A. Berele, A. Regev, A Hook Young diagrams with applications to combinatorics and to representations of Lie superalgebras, Adv. in Math. 64 (1987), 118-175.

[9] N. Bourbaki, Lie groups and Lie algebras, Part 1, Hermann, Paris, 1975.

[10] H. Cartan, S. Eilenberg, Homological algebra, Princeton Mathematics Series, Princeton University, 1956.

[11] J. H. Conway, S. Norton, Monstrous moonshine, Bull. Lond. Math. Soc. 11 (1979), 308-339.

[12] I. Frenkel, J. Lepowsky, A. Meuerman, Vertex Operator Algebras and the Monster, Academin Press, Boston, 1988.

[13] J. Fuchs, B. Schellekens, C. Schweigert, From Dynkin diagram symmetries to fixed point structures, Comm. Math. Phys, 180 (1996), 39-97.

[14] J. Fuchs, U. Ray, C. Schweigert, Some automorphisms of generalized Kac-Moody algebras, J. Algebra 191 (1997), 518-540.

[15] D. B. Fuks, Cohomology of infinite dimensional Lie algebras, Consultant Bureau, New York, 1986.

[16] W. Fulton, J. Harris, Representation Theory: A First Course, Springer-Verlag, 1991.

[17] H. Garland, J. Leopwsky, Lie algebra homology and the Macdonald-Kac formulas, Invent. Math. 34 (1976), 37-76.

[18] V. A. Gritsenko, V. V. Nikulin, Siegel automorphic form corrections of some Lorentzian Kac-Moody Lie algebras, Amer. J. Math. 119 (1997), 181-224.

[19] V. A. Gritsenko, V. V. Nikulin, Automorphic forms and Lorentzian Kac-Moody algebras I, Inter. J. Math. 9 (1998), 153-199.

[20] V. A. Gritsenko, V. V. Nikulin, Automorphic forms and Lorentzian Kac-Moody algebras II, Inter. J. Math. 9 (1998), 201-275.

[21] K. Harada, Modular functions related to the Monster, Lectures given at the conference Moonshine and Vertex Operator Algebra, RIMS, Kyoto Univ., 1994.

[22] J. A. Harvey, G. Moore, Algebras, BPS states, and strings, Nuclear Phys. B463 (1996), 315-368.

[23] J. Hong, J.-H. Kwon, Decomposition of free Lie algebras into irreducible components, J. Algebra. 197 (1997), 127-145. 
[24] Y.-J. Im, Defining relations of generalized Kac-Moody superalgebras, M. S. thesis, Seoul National University (1998).

[25] E. Jurisich, Generalized Kac-Moody Lie algebras, free Lie algebras and the structure of the Monster Lie algebra, J. Pure and Applied Algebra 126 (1998), 233-266.

[26] E. Jurisich, J. Lepowsky, R. L. Wilson, Realizations of the Monster Lie algebra, Selecta Mathematica, New Series 1 (1995), 129-161.

[27] V. G. Kac, Simple irreducible graded Lie algebras of finite growth, Math. USSRIzvestija 2 (1968), 1271-1311.

[28] V. G. Kac, Infinite-dimensional Lie algebras and Dedekind's $\eta$-function, Funct. Anal. Appl. 8 (1974), 68-70.

[29] V. G. Kac, Lie superalgebras, Adv. in Math. 26 (1977), 8-96.

[30] V. G. Kac, Infinite dimensional algebras, Dedekind's $\eta$-Function, Classical Möbius function and the Very strange formula, Adv. in Math. 30 (1978), 85-136.

[31] V. G. Kac, Infinite Dimensional Lie Algebras, Cambridge Univ. Press, 3rd ed., 1990.

[32] V. G. Kac, S.-J. Kang, Trace formula for graded Lie algebras and Monstrous Moonshine, in Representations of groups, B. Allison, G. Cliff (eds), Canad. Math. Soc. Conf. Proc. 16 (1995), 141-154.

[33] V. G. Kac, D. Peterson, Defining relations of certain infinite-dimensional groups, Asterisque, hors serie (1985), 165-208.

[34] V. G. Kac, M. Wakimoto, Modular invariant representations of infinite dimensional Lie algebras and superalgebras, Proc. Natl. Acad. Sci. U.S.A. 85 (1988), 4956-4960.

[35] V. G. Kac, M. Wakimoto, Integrabl highest weight modules over affine superalgebras and number theory, in Lie Theory and Geometry, Progress in Math. 123 (1994), Birkhäuser, Boston, 415-456.

[36] S.-J. Kang, Root multiplicities of Kac-Moody algebras, Duke Math. J. 74 (1994), 635-666.

[37] S.-J. Kang, Generalized Kac-Moody algebras and the modular function j, Math. Ann. 298 (1994), 373-384.

[38] S.-J. Kang, Root multiplicities of graded Lie algebras, in Lie Algebras and Their Representations, Contemp. Math. 194 (1996), 161-176.

[39] S.-J. Kang, Free Lie superalgebras and the generalized Witt formula, RIM-GARC preprint 96-49 (1996).

[40] S.-J. Kang, Graded Lie superalgebras and the superdimension formula, J. Algebra 204 (1998), 597-655.

[41] S.-J. Kang, M.-H. Kim, Free Lie algebras, generalized Witt formula, and the denominator identity, J. Algebra 183 (1996), 560-594.

[42] S.-J. Kang, M.-H. Kim, Dimension formula for graded Lie algebras and its applications, RIM-GARC preprint 96-51 (1996), to appear in Trans. Amer. Math. Soc. 
[43] B. Kostant, Lie algebra cohomology and the generalized Borel-Weil theorem, Ann. Math. 74 (1961), 329-387.

[44] L.-S. Liu, Kostant's formula in Kac-Moody Lie algebras, J. Algebra 149 (1992), $155-178$.

[45] I. G. Macdonald, Affine root systems and Dedekind's $\eta$-function, Invent. Math. 15 (1972), 91-143.

[46] I. G. Macdonald, Symmetric functions and Hall polynomials, Oxford Univ. Press, England, 1979.

[47] M. Miyamoto, A generalization of Borcherds algebra and denominator formula, J. Algebra 180 (1996), 631-651.

[48] R. V. Moody, A new class of Lie algebras, J. Algebra 10 (1968), 211-230.

[49] S. Naito, The strong Bernstein-Gelfand-Gelfand resolution for generalized KacMoody algebras I: The existence of the resolution, Publ. RIMS, Kyoto Univ. 29 (1993), 709-730.

[50] H. Pop, A generalization of Scheunert's theorem on cocycle twisting of color Lie algebras, preprint (1997).

[51] U. Ray, A character formula for generalized Kac-Moody superalgebras, J. Algebra 177 (1995), 154-163.

[52] J. Remmel, The combinatorics of $(k, l)$-hook Schur functions, Contemporary mathematics 34 (1984), 253-287.

[53] M. Scheunert, The Theory of Lie Superalgebras, Lecture notes in Math. 716, Springer-Verlag, Berlin, 1979.

[54] M. Scheunert, Generalized Lie algebras, J. Math. Phys. 20 (1979), 712-720.

[55] H. Weyl, Classical Groups, North-Holland, Amsterdam, 1963. 